\title{
SAND79-8014
}

Unlimited Release

Printed June 1979

\section{DATA ACQUISITION SYSTEM FOR THE HAMILTON STANDARD W2 ELECTRON BEAM WELDER}

\author{
James Hopwood \\ Model Shops and Inspection Division 8423 \\ Sandia Luboratories, Livermore
}

\begin{abstract}
A data acquisition system has been designed which will perform on-line weld-parameter sampling. It is a microprocessor-based program controller and calculator. The parameters sampled are beam current, accelerating voltage, focus-coil current, workpiece rpm, and filament voltage. Sampling in analog form occurs in pre-selected angular-rotation increments from 1 to 9 degrees. There are three data printout options: $A$, all data displayed; B, only out-oftolerance values displayed; and $C$, differences between nominal and sampled values in excess of preselected error bands displayed. A magnetic tape cartridge unit allows long-term data storage and easy retreival.

This report is a manual for system operation. It also describes the design-logic principles, circuitry, and programming--In detail.
\end{abstract}


CONTENTS

Page

Introduction 9

$\begin{array}{ll}\text { System Operation } & 10\end{array}$

Overall Central Unit Design 15

Logic and Circuit Details 17

$\begin{array}{lll}\text { Parameter Measurement Circuitry } & 17\end{array}$

Encoder RPM Logic $\quad 23$

Send Memory Data to Terminal 25

Write Memory Data to Magnetic Tape 30

Read Input Characters From Data Terminal 30

Read Data From Tape and Reload to Memory 30

Data Acquisition and Storage $\quad 35$

Read Memory and Process Data With FPAR 35

Read/Write Memory $\quad 39$

Address Register and Switch Circuit $\quad 41$

Magnetic Tape-Ünit Data and Control Circuit 41

Microprocessor Port Assignments $\quad 41$

Appendix A--Miscellaneous Drawings and Diagrams $\quad 45 / 46$

Appendix B--Program Flow Chart $\quad$ 53/54

$\begin{array}{ll}\text { Appendix C--Detailed Schematics } & \mathbf{5 9 / 6 0}\end{array}$

Program Listing (Microfiche) - Envelope on back page 


\section{ILLUSTRATIONS}

Figure $\quad \underline{\text { Pege }}$

1. Complete Data Acquisition System for the Hamilton 11 Standard Electron Beam Weloicr

2. (a) Front View of Central Processing and Control Unit

(b) Rear View of the Same

3. Sample Printout Showing Operator-Selected Nominal and Error-Band Parameter Values Followed by the Summary of the Same

4. Sample Printout Illustrating Options A, B, and C 14

5. Parameter Sampling Locutions in Welder Circuitry 16

6. Block Diagram--Data Acquisition System 18

7. High Voltage Measurement Circuit 19

8. Beam Current Measurement Circuit 20

9. Focus Current Measurement Circuit 21

10. Filament Voltage Measurement Circuit $\quad 22$

11. Encoder RPM Logic Data Acquisition 24

12. Counter-Encoder Signal Relationship $(X)$ and Linearized 26 Result (1/X)

13. Send Memory Data to Terminal 27

14. Address Register and Switch Circuits $28^{\circ}$

15. RAM Data Select 29

16. Write Memory Date to Magnetic Tape 31 
17. Read Input Characters From Terminal 32

8. Data Terminal Input and Output Shift Registers 33

19. Read Data From Tape and Reload to Memory 34

20. Data Acquisition and Storage 36

21. Schematic for FPAR 37

22. Read Memory and Process Data With FPAR 38

23. (a) Intel 4002-1 RAM Register Map \#0 40 (b) Intel 4002-1 RAM Register Map \#1

24. Magnetic Tape Unit Data and Control Circuit 42

$\begin{array}{ll}\text { 25. Microprocessor Port Assignments } & 43 / 44\end{array}$ 


\section{Introduction}

The precision of the Hamilton Standard Electron Beam Welder is dependent upon five parameters : bear current, accelerating voltage, focus-coil current, workpiece rpm, and filament voltage. Experience has shown that there are often fluctuations in one or more parameters during a weld and that this can affect weld quality. It was for the purpose of monitoring these fluctuations that a data acquisition system was designed.

The system is a microprocessor-based program controller and calculator. On-line sampling in analog form of all five parameters occurs in preselected angular-rotation increments from 1 to 9 degrees. If 1 -degree increments are selected, sampling will occur for each degree of workpiece rotation up to a maximum of 3 revolutions. For 9-degree increments, 27 revolutions are the maximum.

Each sampling is converted to a 3-digit binary coded decimal (BCD) and stored in a $16 \mathrm{~K} \times 4$-bit word on-board read/write memory. After the welding operation, the operator selects one of three output format options, labeled $A$, $B$, and $C$. The stored data are then read from memory, calculations performed, and the appropriate output transmitted to a Texas Instruments Model 733 data terminal. If option A has been chosen, all acquired data are printed in columnar format. If option $B$ has been chosen, the measured values are compared with nominal and error-band values which the operator has entered via the data terminal prior to the welding operation and only those measured parameters which are outside the exror band are printed. If option $\mathrm{G}$ has been chosen, the measured values are, as for $B$, compared with nominal and error-band values, but now the amount that each parameter falls outside the error band is printed. The latter two options permit a quick view of the weld parameters so that the welding engineer can correlate perturbations in the weld with fluctuations in parameters.

To check system operation, there is a compact welder simulation unit which can be readily substituted and used to enter known parameter and error-band values into the system.

An additional storage medium is provided. A magnetic tape cartridge system manufactured by Tri-Data Corporation will record the stored data for future comparisor and possibly manipulation and plotting by a computer. 
System Operation

Figure 1 is a photograph of the complete data acquisition system. Figure 2 shows the central unit, which samples the data and performs the required calculations. It fits in a standard 19-inch rack and is 6 inches high.

Depressing the START button prompts the program to ask the operator (through the terminal printout) for nominal parameter values, error-band values, and the workpiece rotation angle between each data sample. The operator first sees

\section{TYPE NOMINAL}

$\mathbf{R P M}=$

on the printout. The response must be in the form XX.X. Similar requests for nominal values of high voltage (HV), beam current (BC), focus current (FC), and filament voltage (FIL) then follow. After the operation response to "FIL", the program prompts the terminal to write

\section{TYPE ERROR BAND}

RPM =

Upon receiving a response in the form $X . X$, the program requests the remaining error-band values. The final request is

$$
\text { STEP = , }
$$

in response to which the operator types any integer from 1 to 9 , corresponding to the number of degrees between each data sample. The program then prompts the data terminal to print the abbreviation of each parameter followed by a summary of the nominal and error-band values in the same columnar format. Figure 3 shows an example of the entire printout discussed thus far.

If at any time thereafter it is desired to change the operator-selected inputs, activating RESET followed by START will return the.program to the interrogation sequence.

Next, the program waits for a signal from the shaft encoder. Date acquisition and storage in the on-board read/write memory occur after the workpiece has rotated through each commanded increment.

Depressing the STOP button after the welding operation is completed (or, sooner, whenever welder pararueter information is desired) followed by activating the PRINT switch causes the program to ask for the print option: $A, B$, or C. If $A$ is chosen, all data are printed in the same format as the summary of nominal (operator-selected) values immediately above. If $B$ is chosen, only out-oftolerance data are printed and each value is labeled since there is no fixed format. 


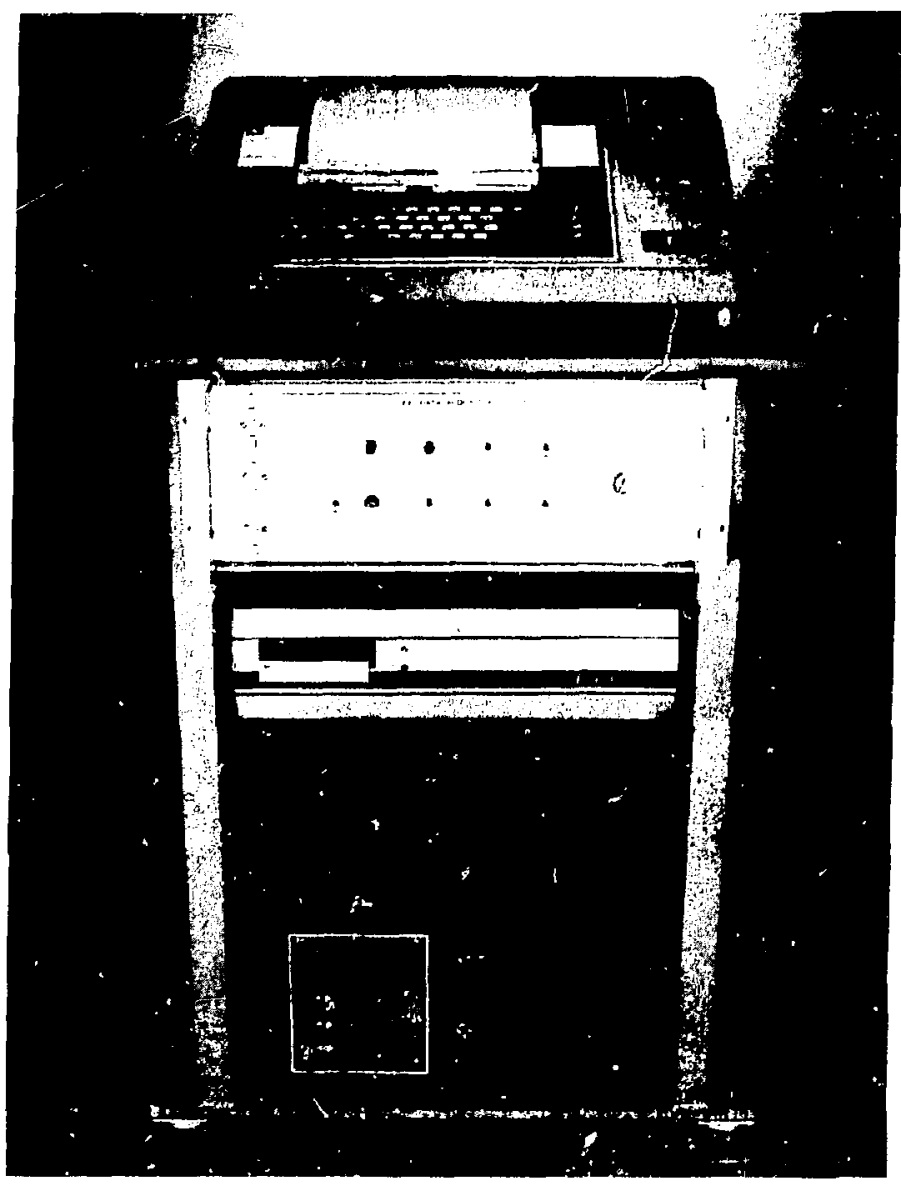

Figure 1. Complete Data Acquisition Systern for the Hamilton Standard Electrón Beam Welder.

(Top to bottom: Texas Instruments Nodel 733 Data Terminal Central Controller and Calculator, Tij-Data Corporation Magnetic 'Tupe Cartridge Unit, and Weluer Simulation Unit) 


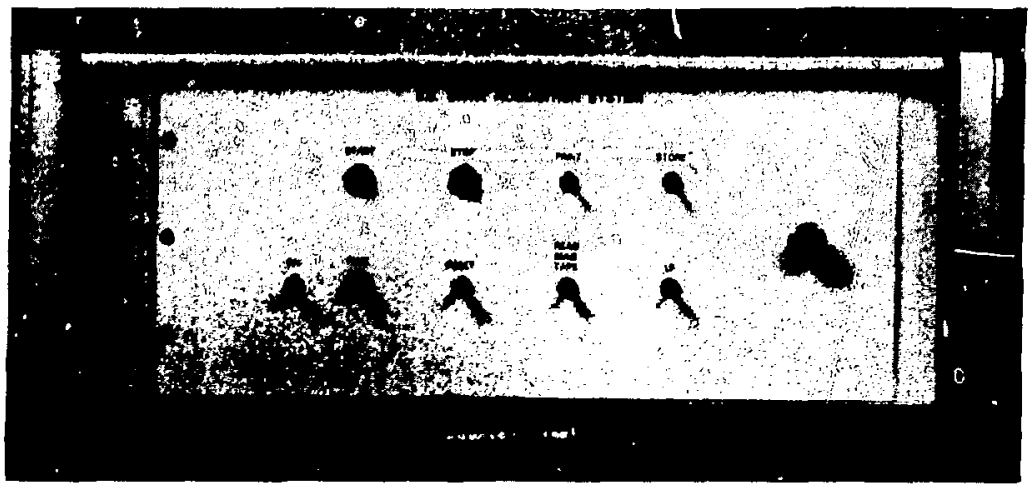

Figure 2a. Front View of Central Processing and Control Unit

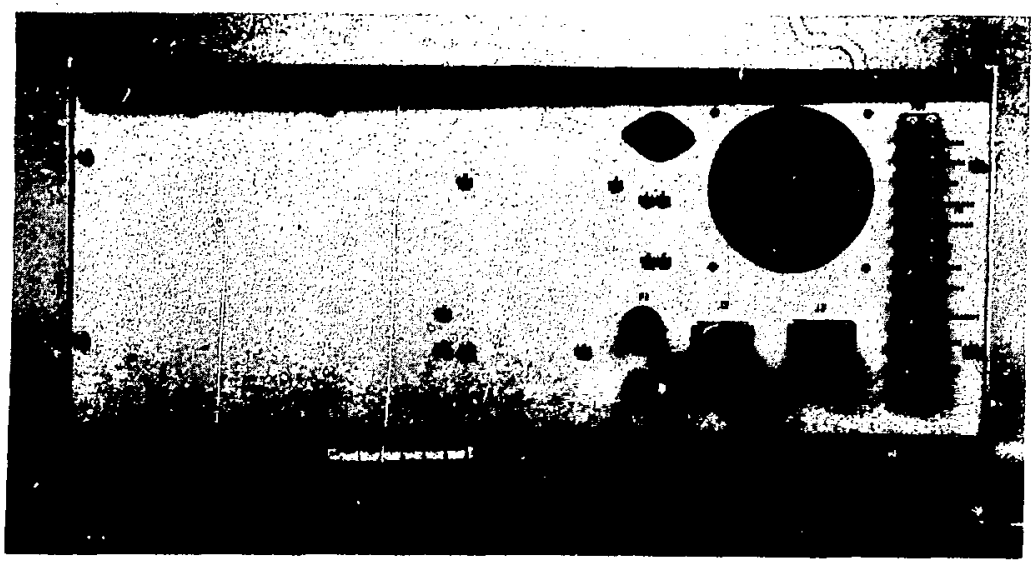

Tigure 2b. Rear View of the Same (Cables from the data terminal and magnetic tape unit attach at $J_{2}$ and $J_{3}$ : respectively. Iines from the welder, or simulation unit, attach to the TB1 array.) 


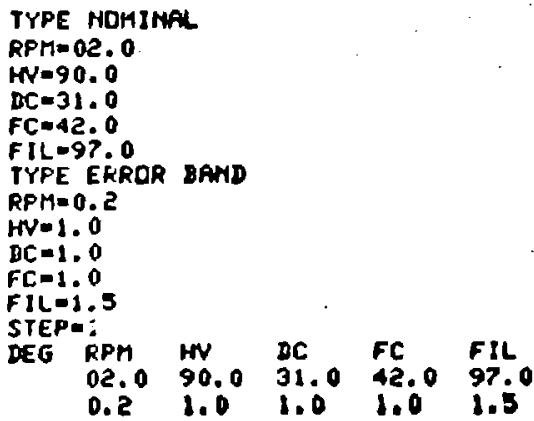

Figure 3. Sample Printout Showing Operatcr-Selected Nominal and Error-Band Parameter Values Followed by the Summary of the Same

If $\mathrm{C}$ is chosen, the terminal prints the magnitude and sign of the parameter fluctuations where they cxceed operator-selected error-band values, again in the same fortnat as the summary of nominal values. Examples are shown in Figure 4. Note that if the step command is 1 (dagree), the numbers in the left-most column are truly the degrees of angular travel. For any cther step command $x=2, \ldots, 9$, the number of degrees of angular travel is found by multiplying by $X$. Since the RAM has a storage capacity for 1088 data points, the number of complete revolutions is given by $1088 \times / 360$ rounded down to the nearest integer.

After 1088 lines are printed, the word END is printed and the program jumps to its initial state. Activation of PRINT allows a difierent print option for the same data.

Activating the RESET switch permits the operator to interrupt one print option before it is completed and, after activating PRINT, choose a new option.

When the system power is switched off, the data sre lost. If it is desired to save the data, activation of the LP switch (load point) causes the tape in the magnetic cantridge unit to advance to a reflective marker which indicates the beginning of the tape. When this has occurred, activation of STORE causes the data to be recorded.

Loading data that are on tape back into the central unit is accomplished activating LP followed by READ MA? TAPE.

If the operator wishes to verify correct system operation, the welder simulation unit can be used to feed definite parameter values into the central unit. Its operation is self-evident from front-panel controls. 
DEG $\begin{array}{lllll}\text { RPH } & \text { HY } & \text { DC } & \text { FC } & \text { FIL } \\ 02.0 & 90.0 & 31.0 & 42.0 & 97.0 \\ 0.2 & 1.0 & 1.0 & 1.0 & 1.5\end{array}$

TYPE FORTAT CODE A

TYPE FORTIAT CODE

$\begin{array}{lllllll}001 & 02.0 & 91.0 & 29.7 & 10.9 & 97.0 \\ 002 & 02.1 & 91.0 & 29.7 & 10.9 & 97.0 \\ 003 & 02.0 & 91.0 & 29.7 & 10.9 & 97.0 \\ 004 & 02.0 & 91.0 & 29.7 & 40.9 & 97.0 \\ 005 & 02.0 & 91.0 & 29.7 & 40.9 & 97.0 \\ 005 & 02.0 & 91.0 & 29.7 & 41.0 & 97.0 \\ 007 & 02.0 & 91.0 & 29.7 & 40.9 & 97.0 \\ 003 & 02.0 & 91.0 & 29.7 & 40.9 & 97.0 \\ 003 & 02.0 & 91.0 & 29.7 & 10.9 & 97.0 \\ 010 & 02.0 & 91.0 & 29.7 & 40.9 & 97.0 \\ 011 & 02.0 & 91.0 & 29.7 & 11.0 & 97.0 \\ 012 & 02.0 & 91.0 & 29.7 & 40.9 & 97.0 \\ 013 & 02.0 & 91.0 & 29.7 & 40.9 & 97.0 \\ 014 & 02.0 & 91.0 & 29.7 & 40.9 & 97.0\end{array}$

$001 \mathrm{BC}=29.7 \mathrm{FC}=40.9$ 002 BCm 29.7 FC $=40.9$ 003 BCm29.7 FC=10.9 $004 \mathrm{BC}=29.7 \quad F C=40.9$ $005 B C=29.7 \quad F C=40.9$ $006 \mathrm{BC}=29.7 \mathrm{FC}-10.9$ 007 BC-29.7 FC-40.9 008 BC $=29.7$ FC $=40.9$ 009 BC $=29.7 \mathrm{FC}=40.9$ 010 BC $=29.7 \mathrm{FC}=40.9$ $011 \mathrm{BC}=29.7 \mathrm{FC}=40.9$ 012 DC-29.7 FC=40.9

TYPE FoRmat 'CODE C:

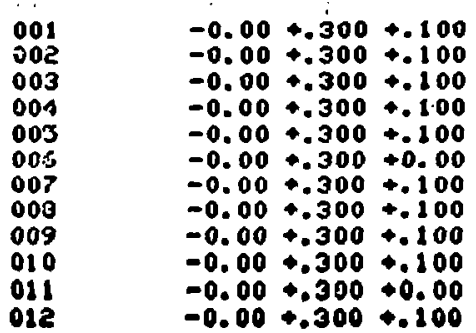

Figure 4. Sample Printout Illustrating Options A, B, and C 


\section{Overall Central Unit Design}

The entire system is contained on 20 printed-circuit cards. Three cards encompass the programmed logic system (PLS 443) containing the microprocessor and stored program. These were purchased from Pro-Log Corporation of Monterey, California. The remaining 17 cards were designed and fabricated in-house. Four of these cards contain the analog-to-digital converters and operational ampliffers which interface and coiditicn the analog signals from the welder prior to their input to the A-to-D converter. This conditioning includes (1) buffering to prevent loading of the welder, (2) filtering to remove the 60cycle and 180-cycle ripple component present in the welder circuitry due to the high-voltage power supply and 3-phase motor-generator, and (3) amplification to bring the signal within the working range of the converter $(0-10 \mathrm{~V})$. The four cards to the left of the PLS contain the 16K $\times$ 4-bit RAM which stores all measured parameter data acquired during the previous weld. One card contains the National Samiconductor 57109 floating-point arithmetic unit (FPAR) and associated circuitry, which performs the arithmetic operation and gives the microprocessor system great calculating power. ${ }^{*}$ The remaining eight cards contain the logic circuitry necessary to interface to the magnetic tape unit, data terminal, front-panel switches, input-dala multiplexers, and miscellaneous control circuitry.

Figure 5 is a simplified schematic of the beam current and focus coil circuitry within the welder showing the modifications which permit parameter sampling. To measure the beam current, the circuit is split at wire $\$ 420$ at $61 \mathrm{~TB}$, and a 100-ohm precision resistor inserted. The voltage developed across the resistor is used to measure the beam current. For example, $4 \mathrm{~V}$ corresponds to $40 \mathrm{~mA}$. This voltage also drives a digital voltmeter mounted within the operator's console.

To measure the accelerating voltage the circuit is split at wire \#421 at $61 \mathrm{~TB}$, and a 750-ohm precision resistor inserted. By inserting this resistor in the 75-megohm 2-MA bleeder stmng, the voltage developed across the resietor becomes directly proportional to the accelerating voltage. For example, $1.5 \mathrm{~V}$ (resistor) corresponds to $150 \mathrm{kV}$ (accelerating voltage).

The focus coil current is measured by means of a $1-\mathrm{chm}$ precision resistor between wire \#224 and \#225 in the focus-coil power supply. Thus 1 amp corresponds to 1 volt.

For complete details on the FPAR see D. C. Macmillan, "A Floating Point Arithmetic Unit for Pro-Log Based Control Systems," Sandia Laboratories Report SAND77-8041, 1977. 


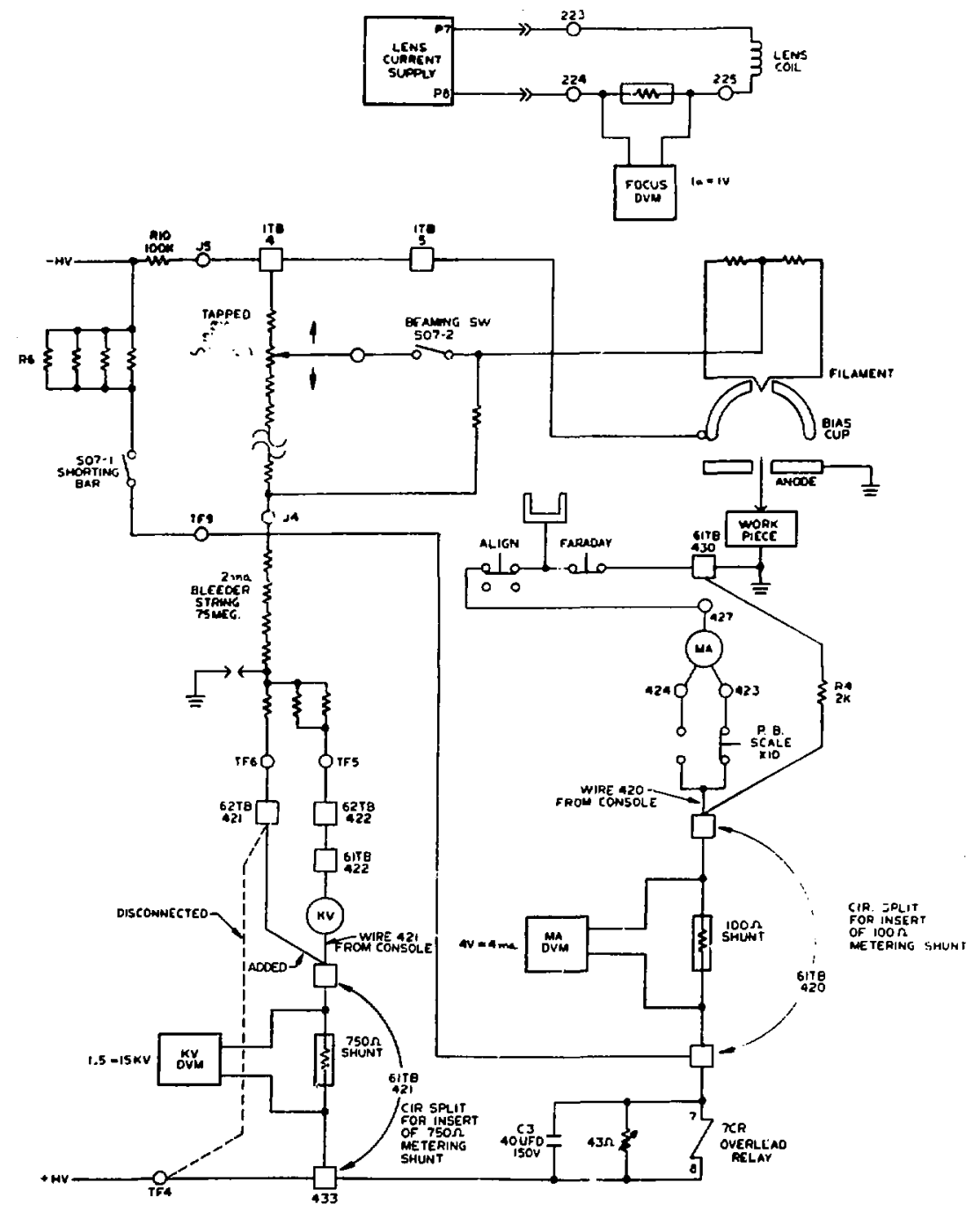

Figure 5. Parameter Sampling Locations in Welder Circuitry 
Figure 6 is a block diagram of the data acquisition system. The program is stored in read-only memories (ROMs) within the program logic system. A total of 16 Intel 1702 ROMs contain almost 4,000 instructions. The PLS has 8 ports; 2 are wired for input and 6 for output.

A large amount of data must pass into the microprocessur through inport 0 . To accomplish this, a series of 3 cascaded multiplexers are wired to the data sources. The program selects specific data by first activating the appropriate multiplexer address lines and then reading inport 0 .

Prior to reading in parameter values, the program issues a Start Conversion command. The analog data are convcried to an equivalent BCD number in 17 microseconds. The program then immediately reads the $\mathrm{BCD}$ data and stores them in the read/write memory. The FPAR (which performs all calculations), read/write memory, magnetic tape, and data terminal are addressed at appropriate tic.es by command lines from the output ports.

The Texas Instrument Model 733 terminal sends and receives chalucters at a $300 \mathrm{cps}$ baud rate and prints data in 1 of 3 formats as explained previously.

\section{Logic and Circuit Details}

\section{Parameter Measureinent Circuitry}

Figures 7-10 show the detail schematic of the high voltage, beam current, focus current, and flament voltage measurement and signal conditioning circuits, respectively. The first stage is an operational amplifier connected in a differential configuration to act as a buffer between the data acquisition system and the welder circuit from which the sampling is made. A differential form is necessary because the location of the paramter sampling is above Earth ground and, elso, it is necessary to prevent loading of the welder circuitry. The output of the buffer enters a Sallen-Key unity-gain, low-pass filter to remove the 60- and 180-cycle ripple present in the welder beam current circuitry. The filter output is a ripple-free dc voltage which is applied to an amplifier with a gain sufficient to bring the voltage level to the working range of the analog/ digital converter. The high voltage and focus current measurement circuit require a gain of 10 whereas the beam current circuit has a gain of 1. The filament voltage measurement circuitry is unique and will be explained later.

The analog/digital converter requires an input voltage of 0 to $+10 \mathrm{~V}$. After conversion, the output is a 3-digit BCD representation of the analog input. After receiving a Start/Convert signal, conversion is complete in 17 microseconds. The converter is Mode] \#ADC-L12D2B 2 and is manufactured by Datel Corporation. 


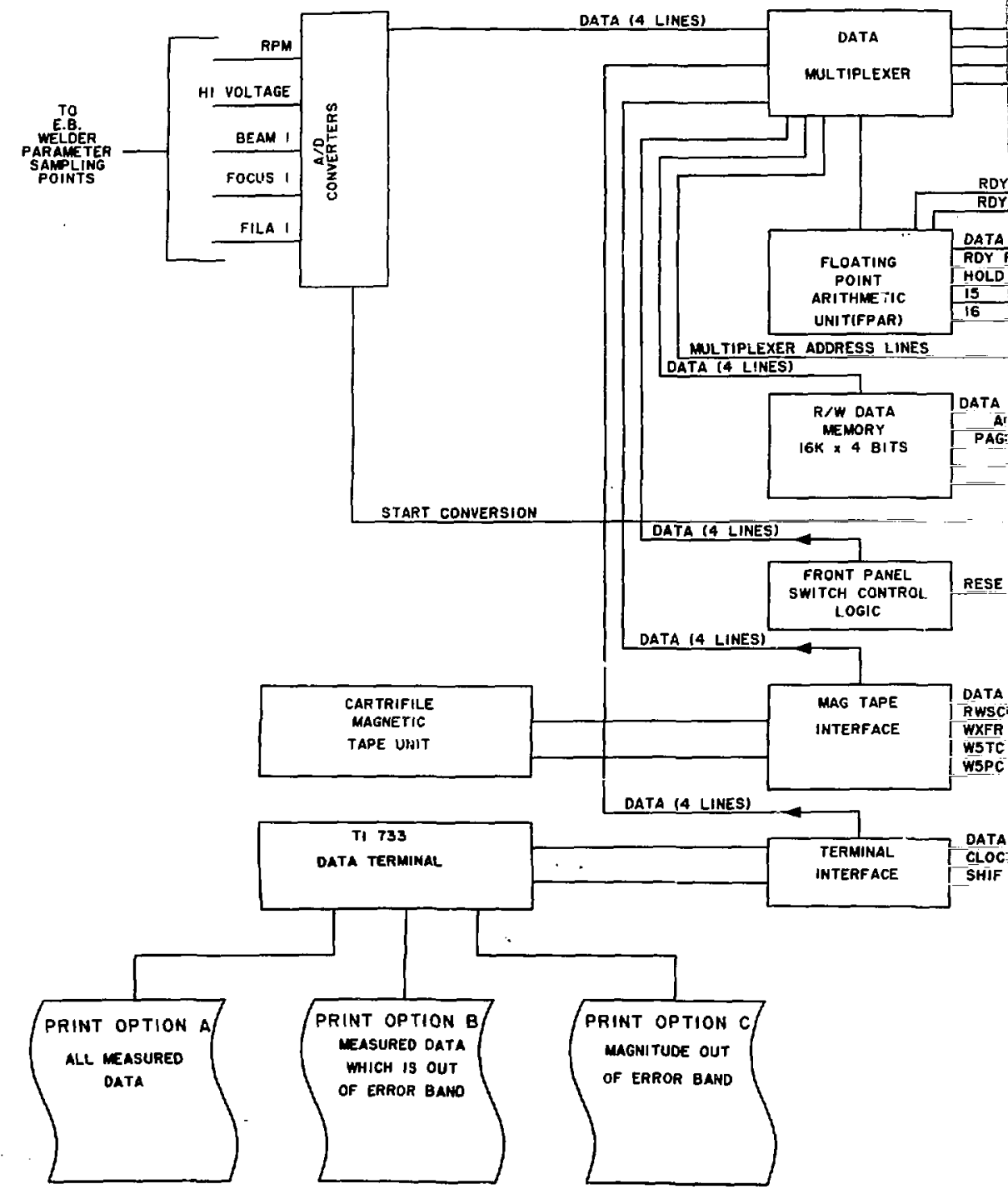




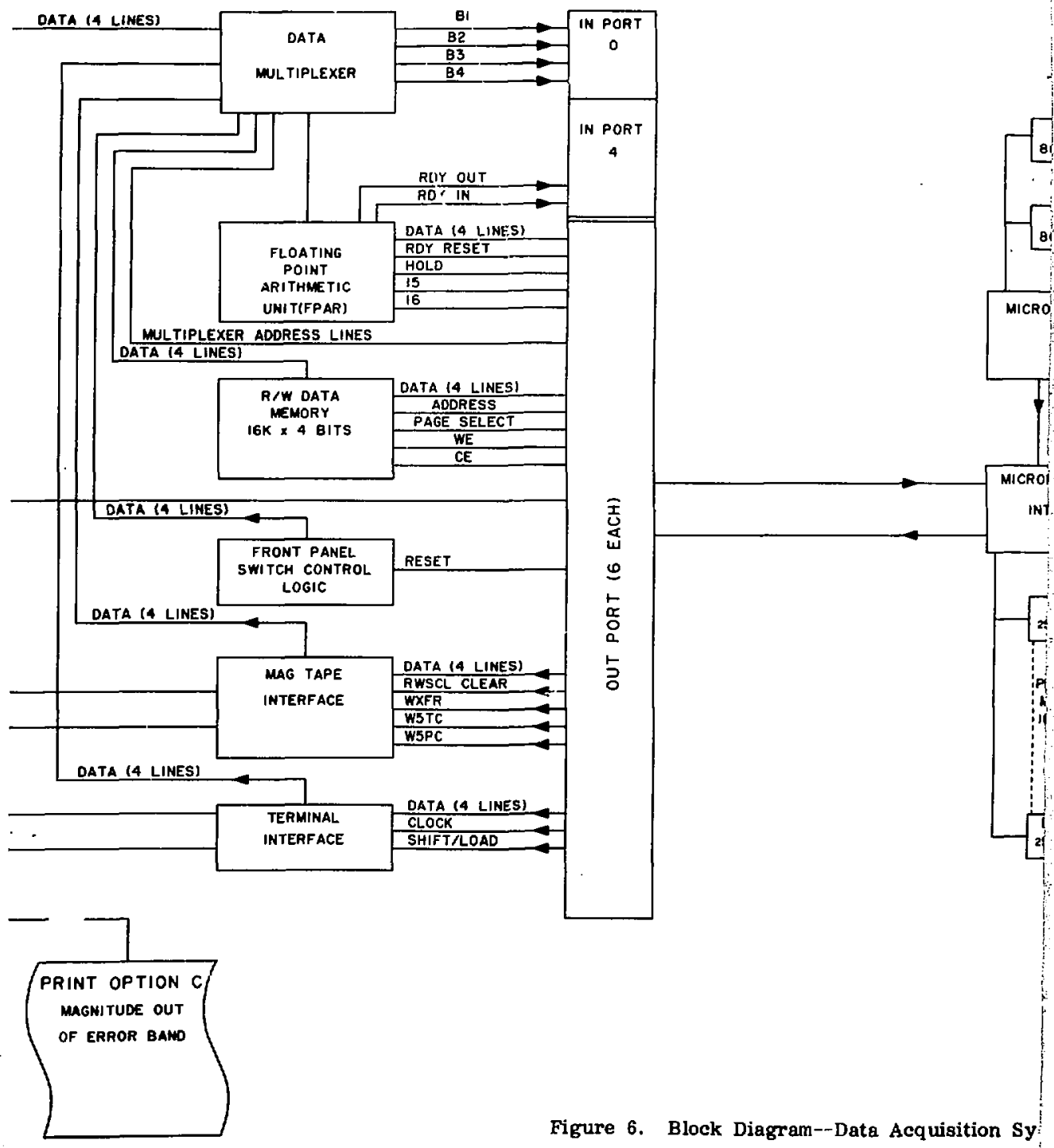




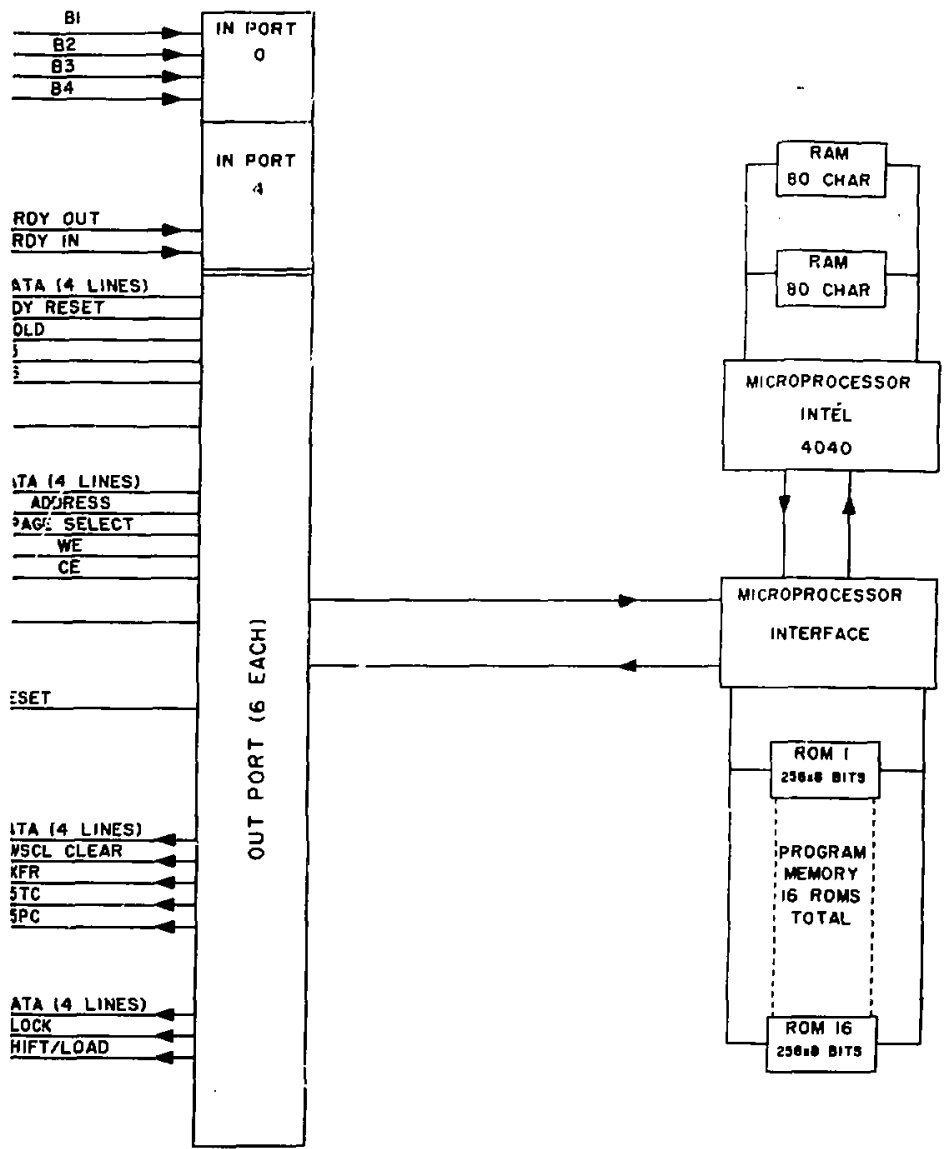

Figure 6. Block Diagram--Data Acquisition System 


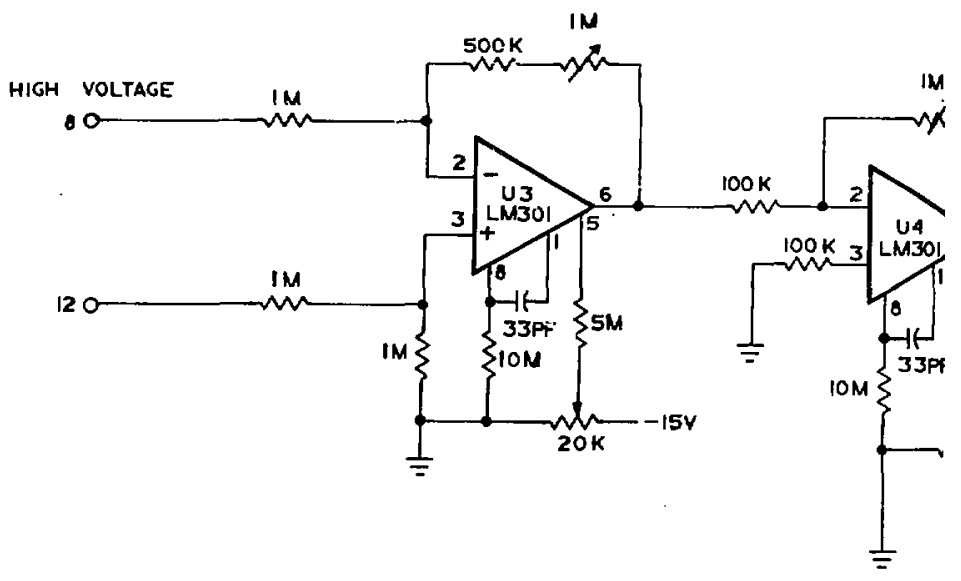

Figure 7. High Volt 


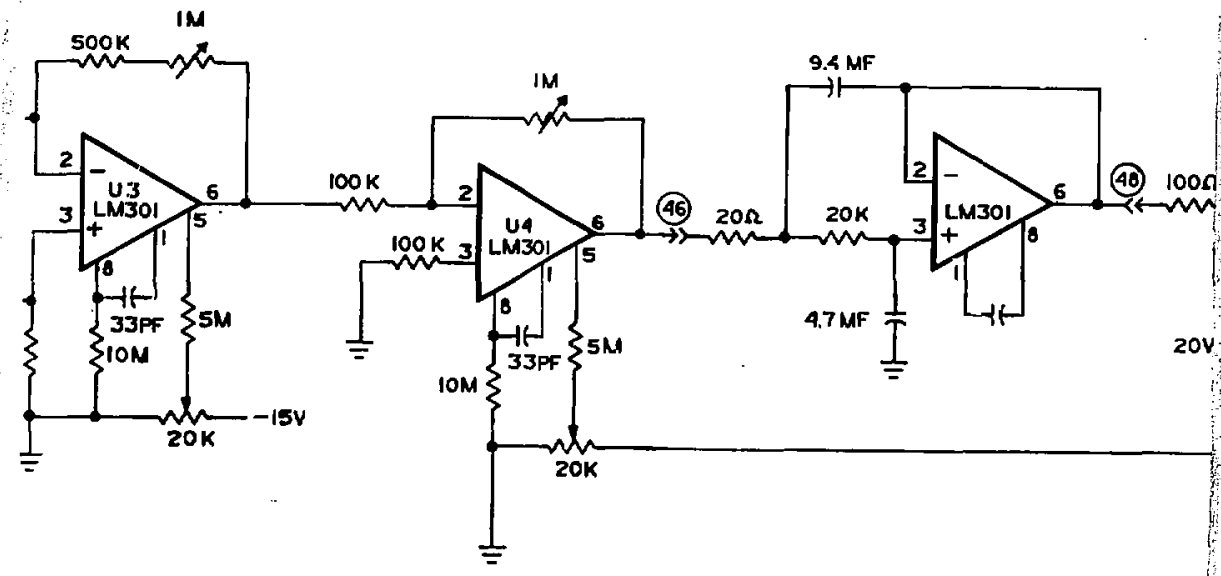

Figure 7. High Voltage Measurement Circuit 


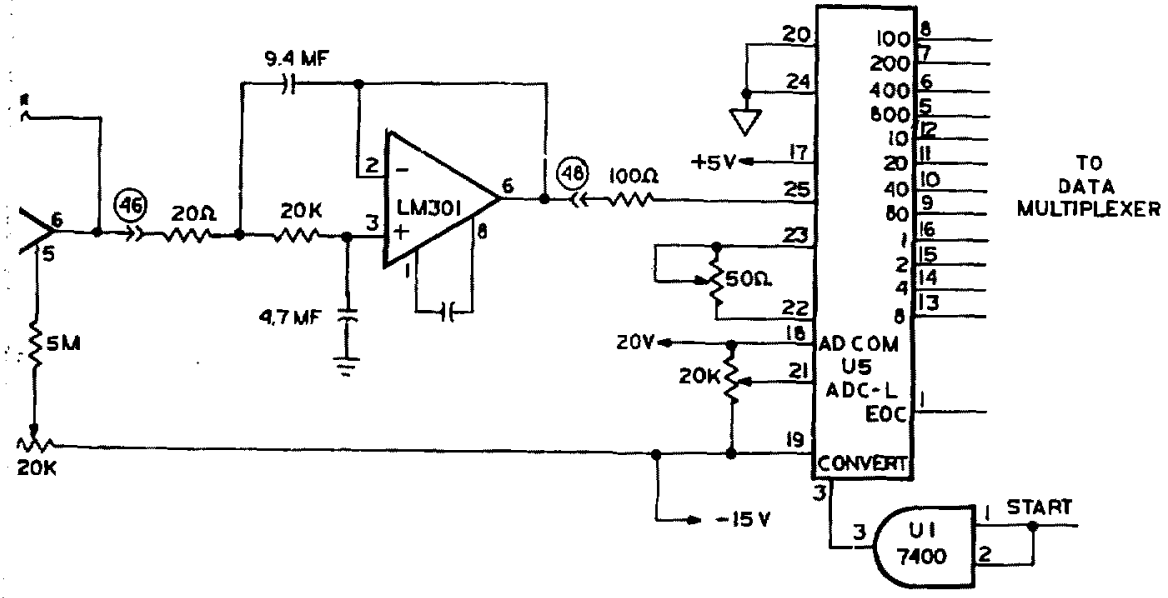

age Measurement Circuit 


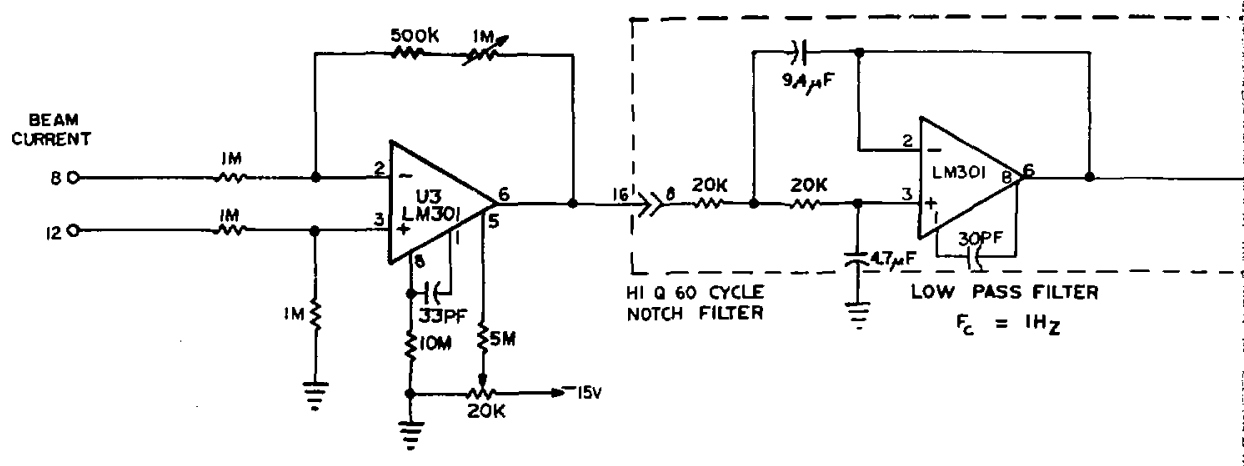

Figure 8. Beam

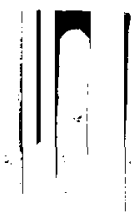




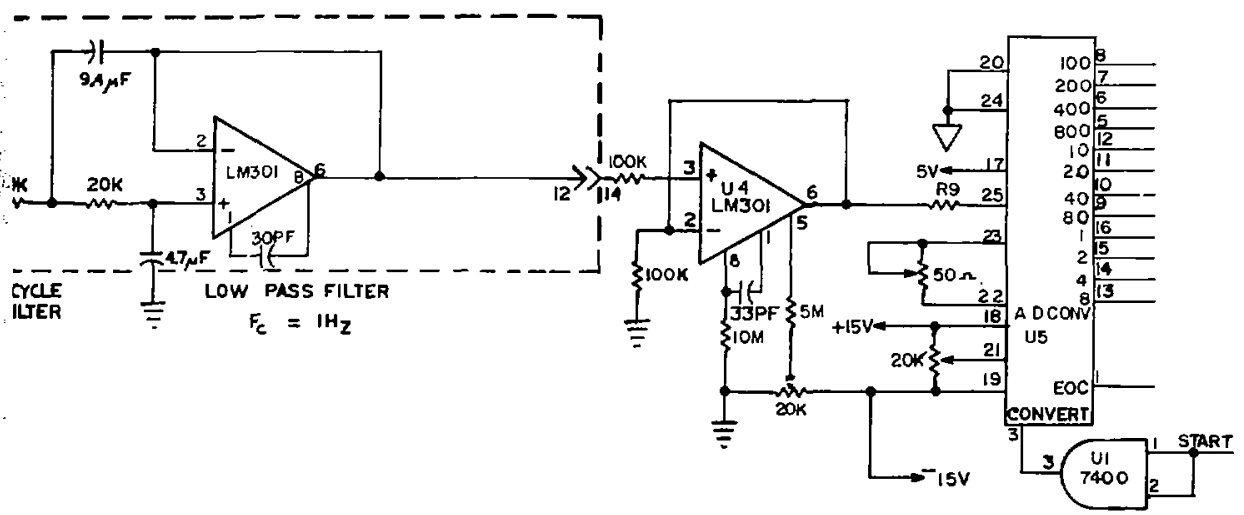

Figure 8. Beam Current Measurement Circuit

TO DATA MULTIPLEXER 


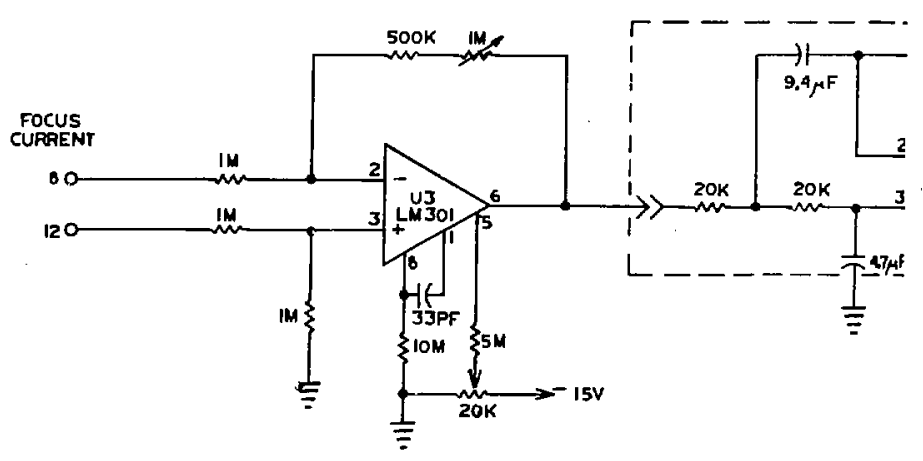

Figure 9. Foc 


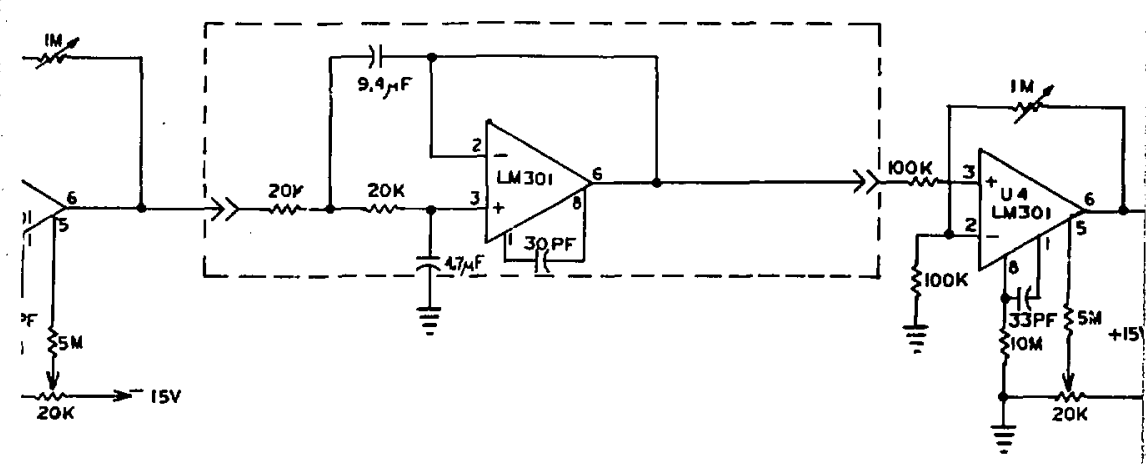

Figure 9. Focus Current Measurement Circuit

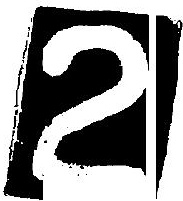




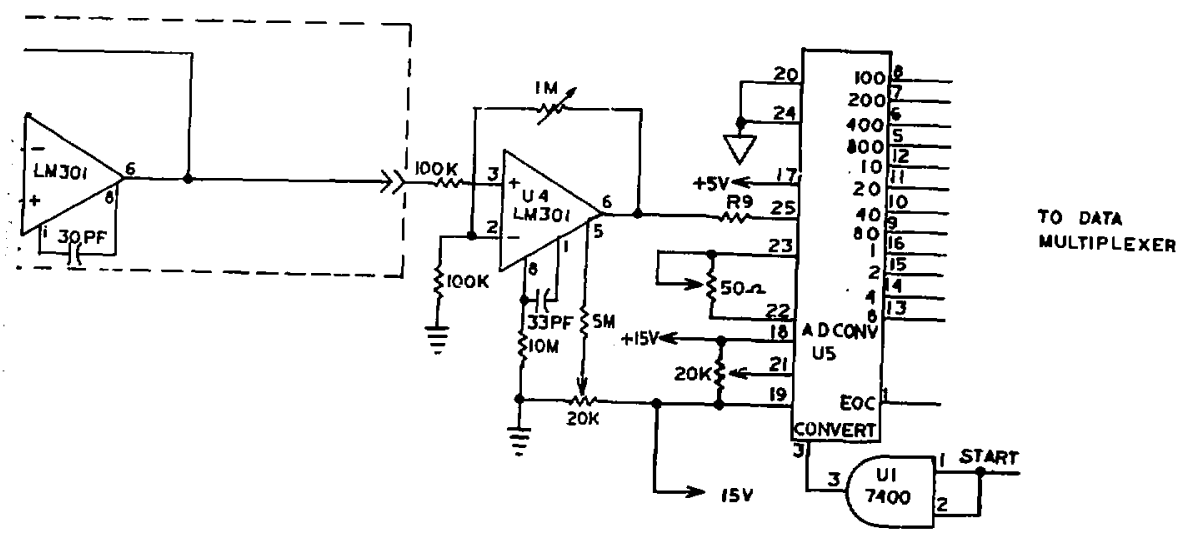

18 Current Measurement Circuit 


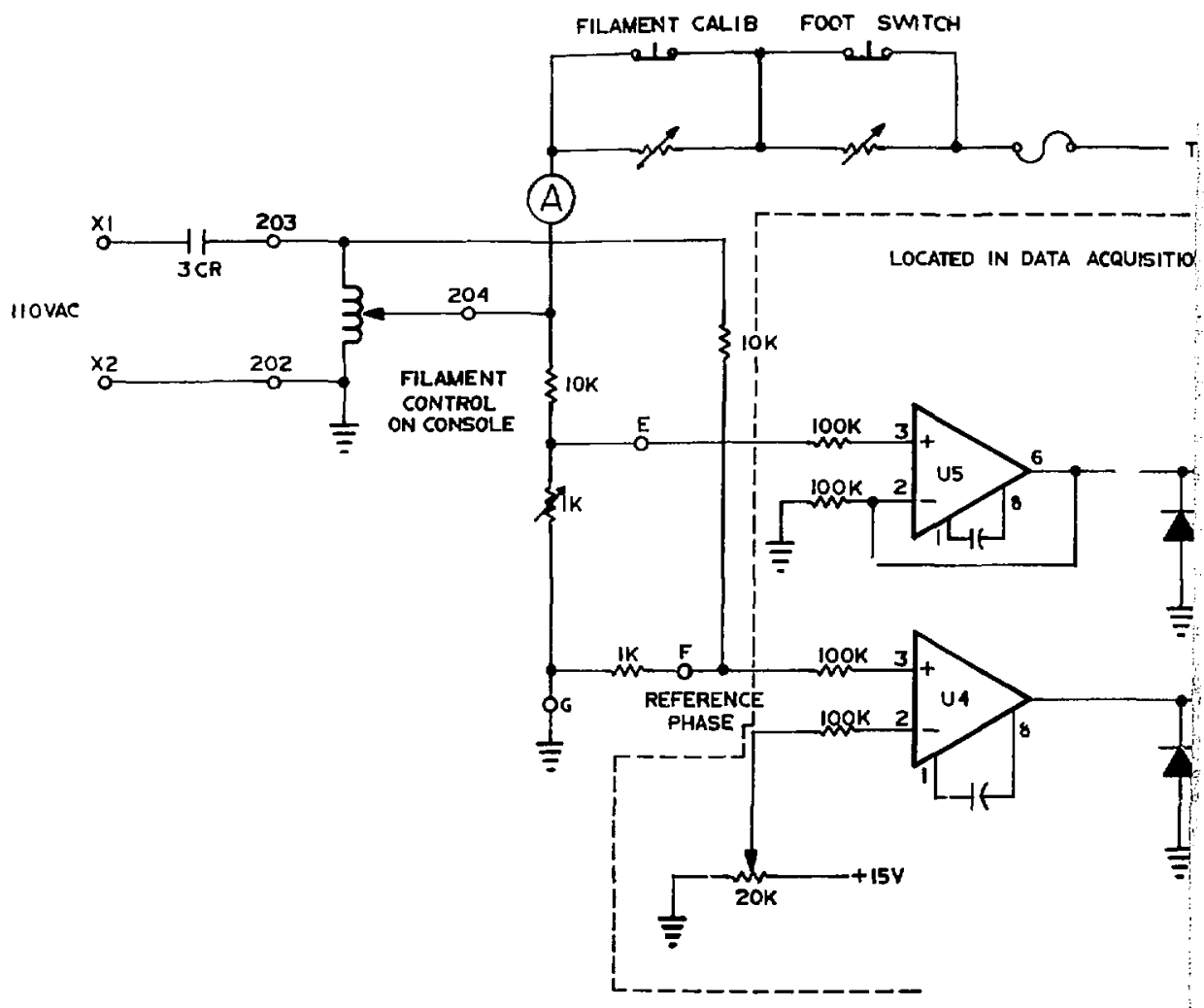

Figure 10. Filament Voltage Measurem! 


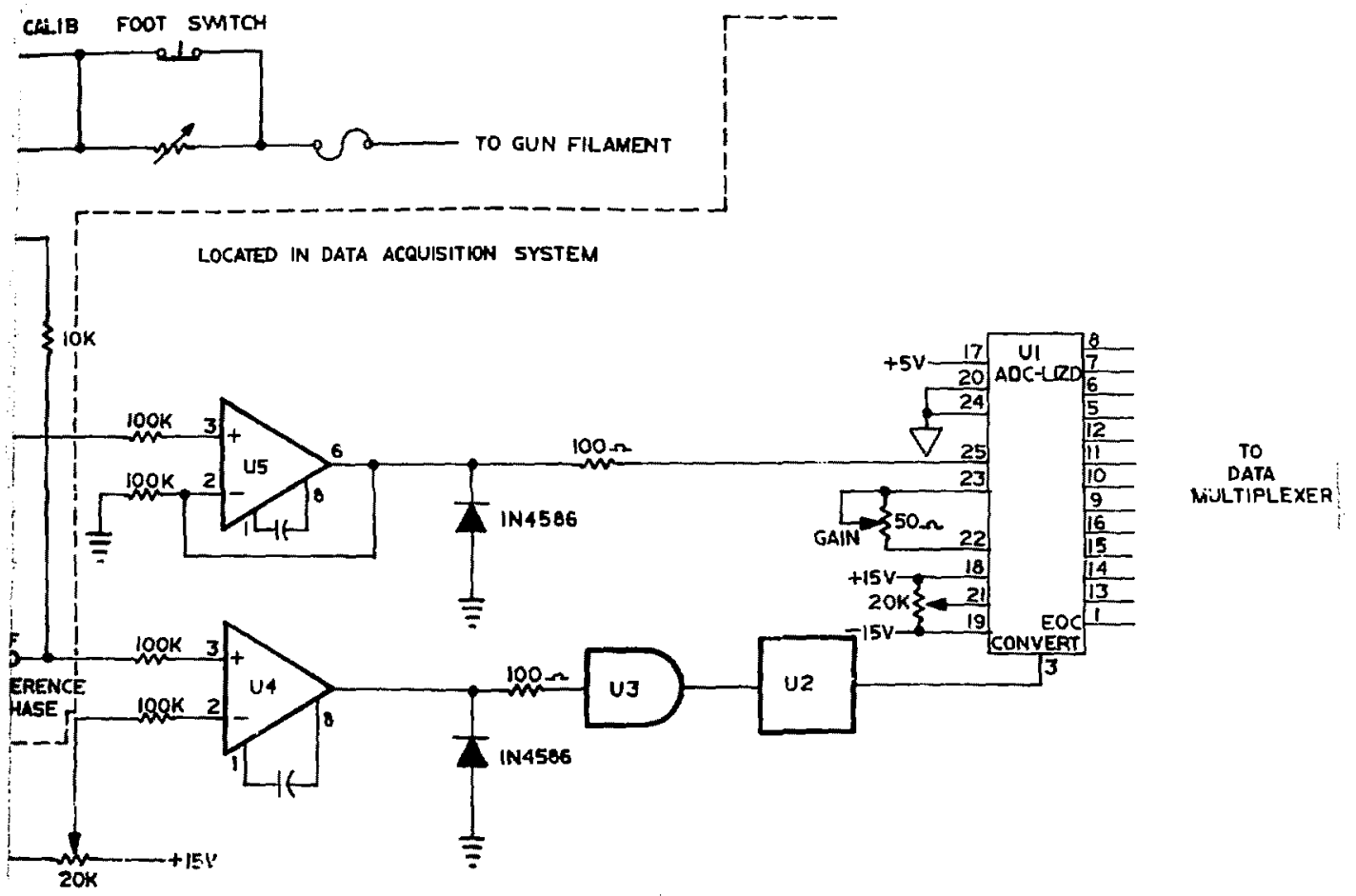

Figure 10. Filament Voltage Measurement Cireuit 
Filsment Voltage Measurement Circuitry--The degree of heat of the electron beam zun filament is directly related to the position of an auto-transformer mounted in the operator's console. This transformer is supplied with 100 volts ec. The filament measurement circritry (Figure 10) samples the voltage at the center tap of the auto-transformer. Any change in the ac supply to the filamerit is thus detected.

The measurement circuit consists of 2 operational amplifiers, $U 4$ and U5. U4 re'. ives the reference phase which sets the point at which the voltage from the tapped transformer is sampled. The voltage divider, consisting of a $10 \mathrm{k}$ $\mathrm{chm}$ ar. $\mathrm{lk} \mathrm{ohm}$ resistor, supply a voltage to the input of the reference amplifier. The point at which the amplifier conducts is set by adjusting the 20k ohm potentiomuter connected to the inverting input of $U 4$. The input to the other amplifier U5 is also from a voltage divider connected to the slider of the autotransformer. Diodes are connected at the output of each amplifier to clip the negative going half of the ac cycle. When $U 4$ conducts it eauses a Start/Convert signal, converting the voltage appearing at U5 output. In this manner the same angular part of ti.e ac cycle is sampled each time.

\section{Encoder RPM Logic}

The purpose of the encoder RPM logic is to sense the presence of output gignals from the encoder and to convert the time between encoder signals to a digital number. To obtain the RPM, the digital number, stored in a 3-stage decade cointer, is processed by the microprocessor system by its taking the reciprocal of the number and multiplying by 1000 .

A 4-bit binary counter is connected to receive the encoder output signal. (See Figlire 11.) It counts down from 4, and when it reaches 0 the one-shot U1-A triggers, reloading the $A$ back into the counter. This division is necessary because the fixture used to hold the workpiece has a 4-to-1 gear reduction from the nounting location of the encoder.

When the counter has reached 0 , triggering one-shot $U-1 A$, flip-flop $U 3$ 1s toggled, enabling the $500 \mathrm{kflz}$ oscillator which is made up of gate U4, resistors $R 3$ and R4, and capacitor C5. The oscillator is connected to the input of a 74C195 4-stage shift register.

The output of one-shot U1-A triggers one-shot U1-B which is connected to the shift/load input of the 74C195. Bit 1 (pin 4) is connected to $10 \mathrm{~V}$ (logic 1). Because of the 5-microsecond delay effected by one-shot U1-A, the shift/load signal arrives 0.5 microseconds after the first transition of the clock. The clack aignal now shifts a bogical 1 progressively through on each clock signal. When shifted to the bit 2 position, a load pulse is generated, loading the state of each decade counter U6, U7, and U 8 into the 3-stage storage registers U9, U10, and U11. When shifted the fourth time, another clock pulse is generated which loads all zero's in U6, U7, and U8--effectively a resetting action. 

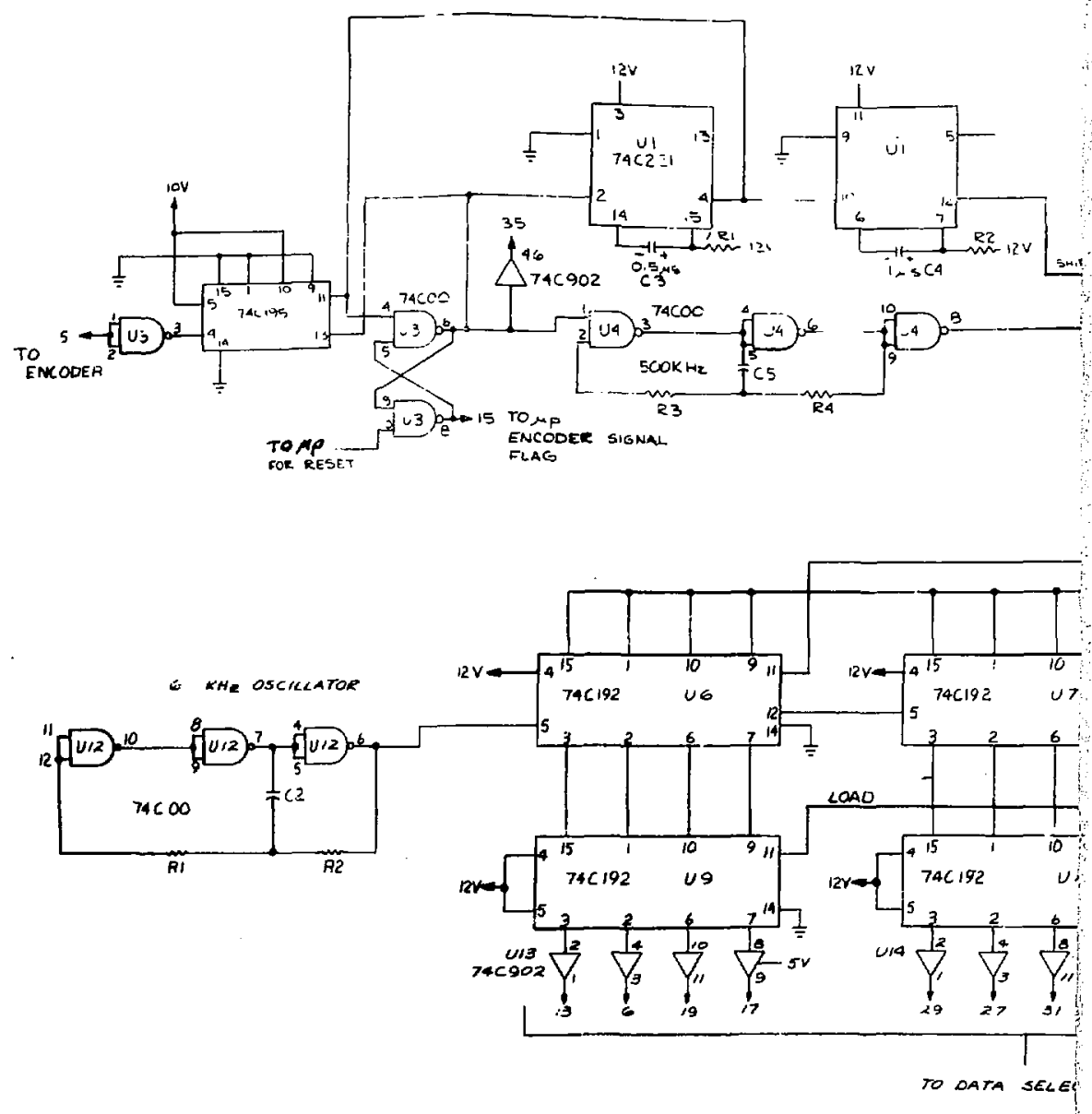

Figure 11. Encoder RPM Logic Data Acquisitio 


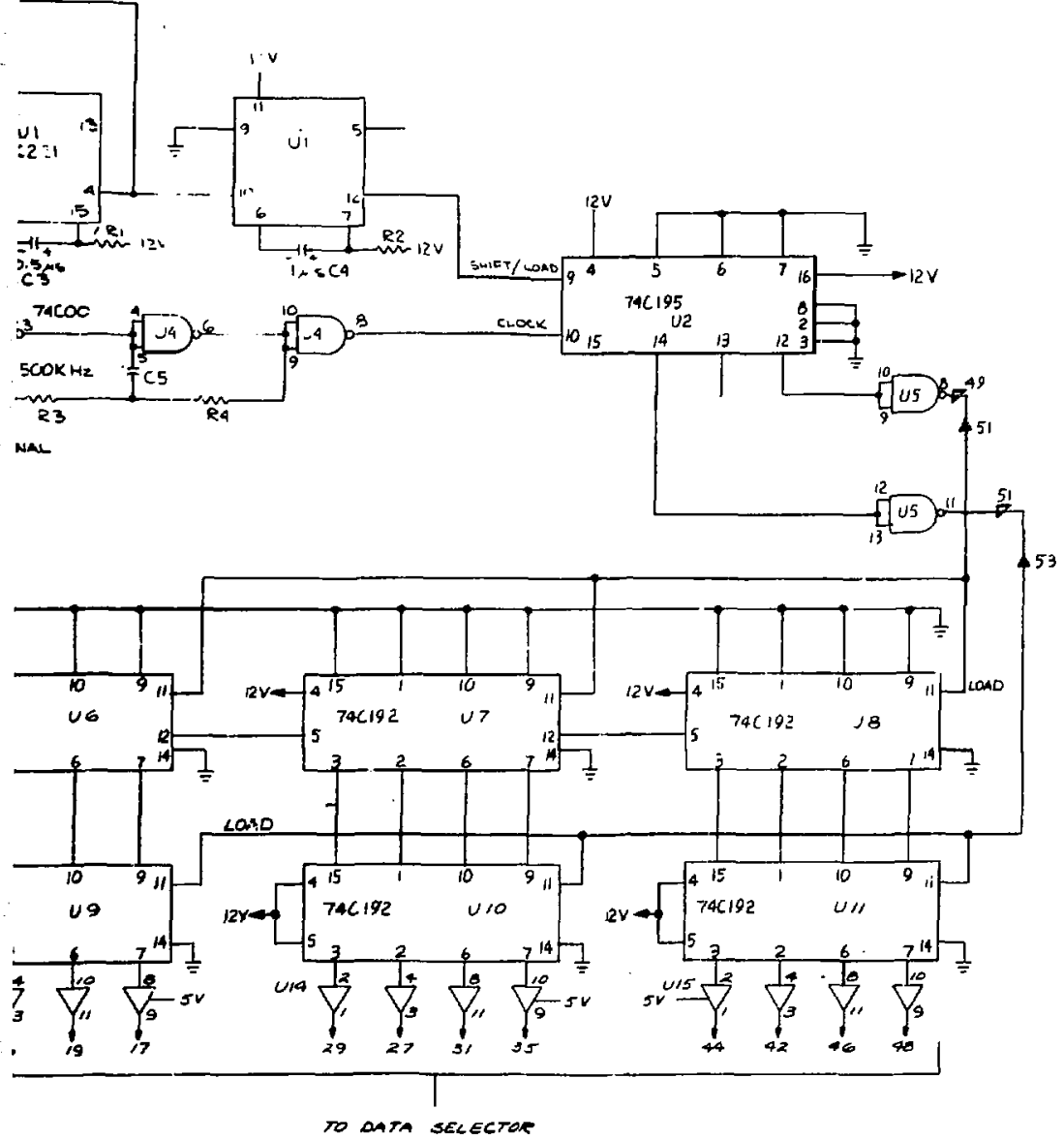

Encoder RPM Logic Data Acquisition 
Registers U9, U10, and U11 now contain the number of counts allowed to enter the 3-stage counter between 2 encoder signals.

The clock oscillator operating the counter is made up of U12, R1, R2, and C2. The frequency of oscillation is $6 \mathrm{kHz}$ (166.6 microseconds period). This frequency causes the number in the counter to be inversely proportional to the time between the encoder's signals. For example, if the encoder is turning at 10 RPM and an output signal occurs each degree of rotation, then

$$
\begin{aligned}
& \frac{\text { RPM } \cdot 360}{60}=\frac{\text { signals }}{\text { sec }} \longrightarrow \frac{3600}{60}=60 ; \\
& \frac{1}{\text { signal } / \mathrm{sec}}=\text { Period } \\
& \frac{\text { Period }}{\text { Clock Period }}=\text { Count in Register } \rightarrow \frac{1}{60}=0.0166 \mathrm{sec} ; \\
& 166 \times 10^{-6}=100 .
\end{aligned}
$$

The counter is an inverse function of the time between encoder signals. To linearize the curve, the microprocessor and FPAR combination finds the reciprocal as follows (see Figure 12):

$$
\frac{1}{100} \times 1000=10 \mathrm{RPM}
$$

The minimum RPM is 1 . If it is lower, the 3-stage counter will overflow. Maximum RPM is 30 . This limitation is due to the time needed for the microprocessor to fetch and store parameter values before returning to detect another encoder signal.

Send Memory Date to Terminal

This mode of operation fetches data from the read/write memory, performs calculations if necessary, and send these data to the Texas Instrument Model 733 terminal for printout. A schmatic diagram is shown in Figure 13.

The program, through outport 7 , initializes the memory address register (see Figure 14). The 12 address lines A-0 through A-11 select 2 of 4000 locations in memory to be interrogated. To address higher order locations, the appropriate chip enoble $(\overrightarrow{\mathrm{CE}})$ line is activated. Since the memory is composed of 4 printed circuit cards, or pages, eech page containg $4 \mathrm{~K}$ of memory. $\overline{C E 1}$ 


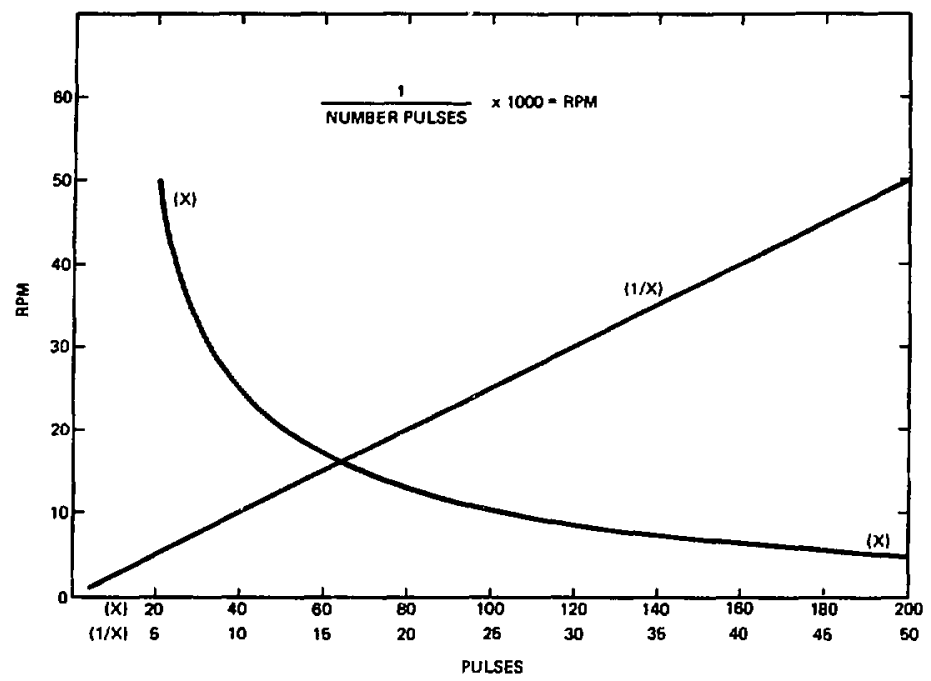

Figure 12. Counter-Encoder Signal Relationship (X) and Linearized Result (1/X) 


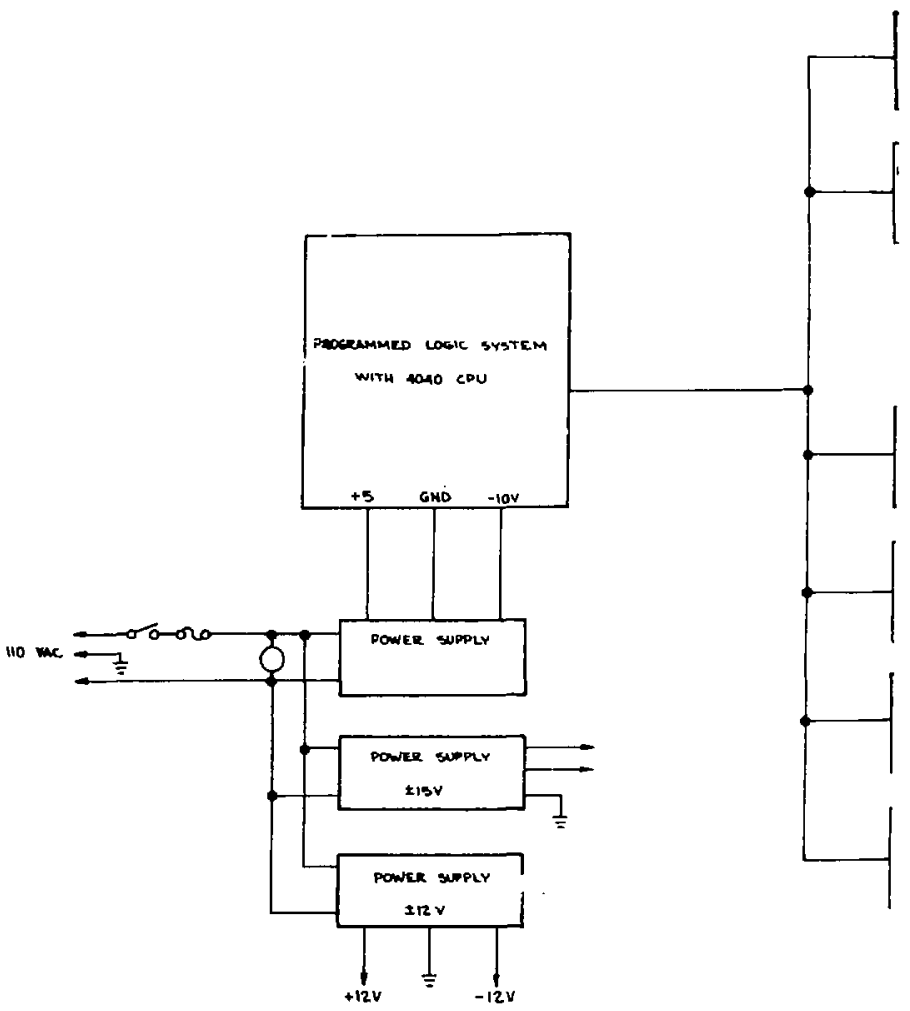

Figure 13 


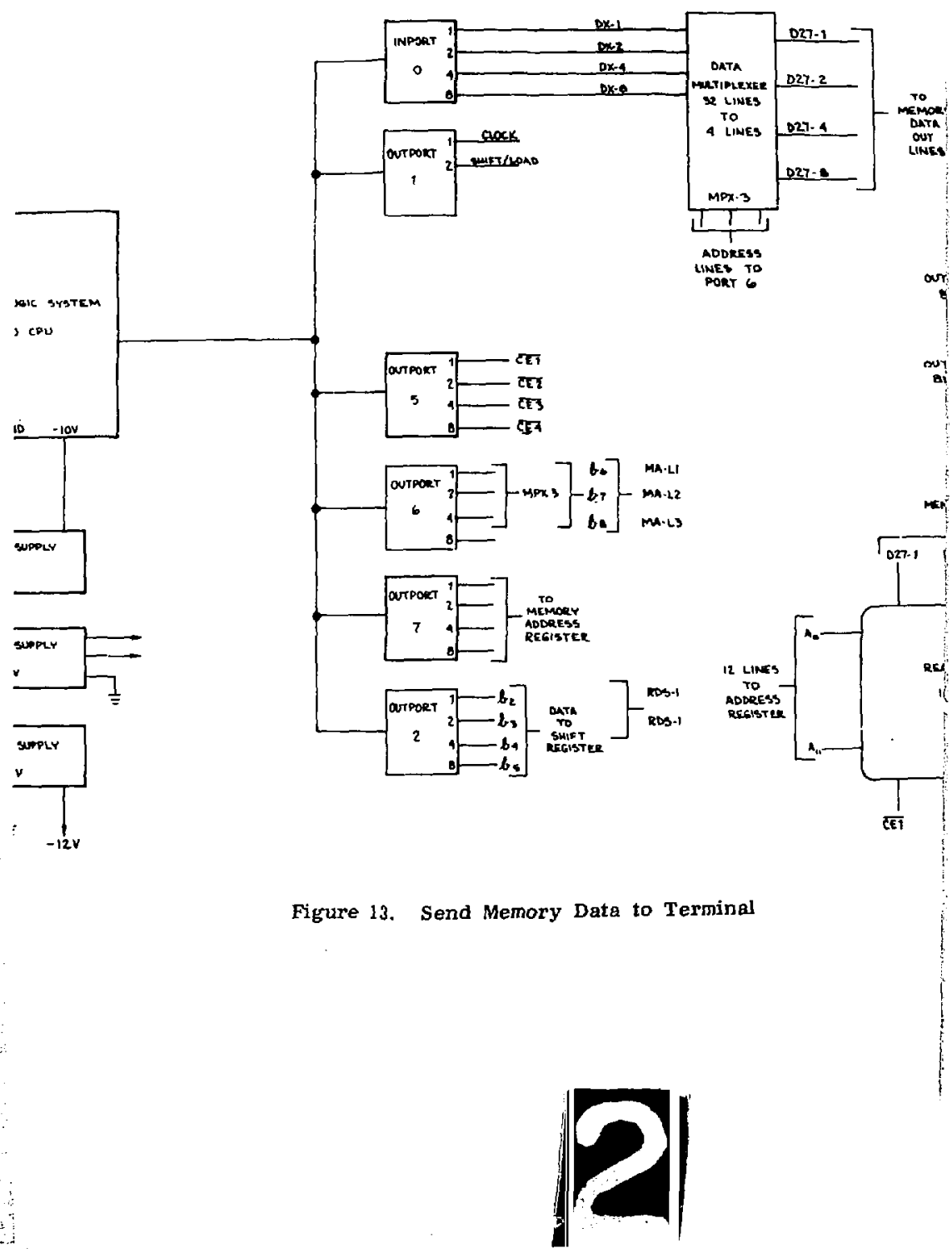




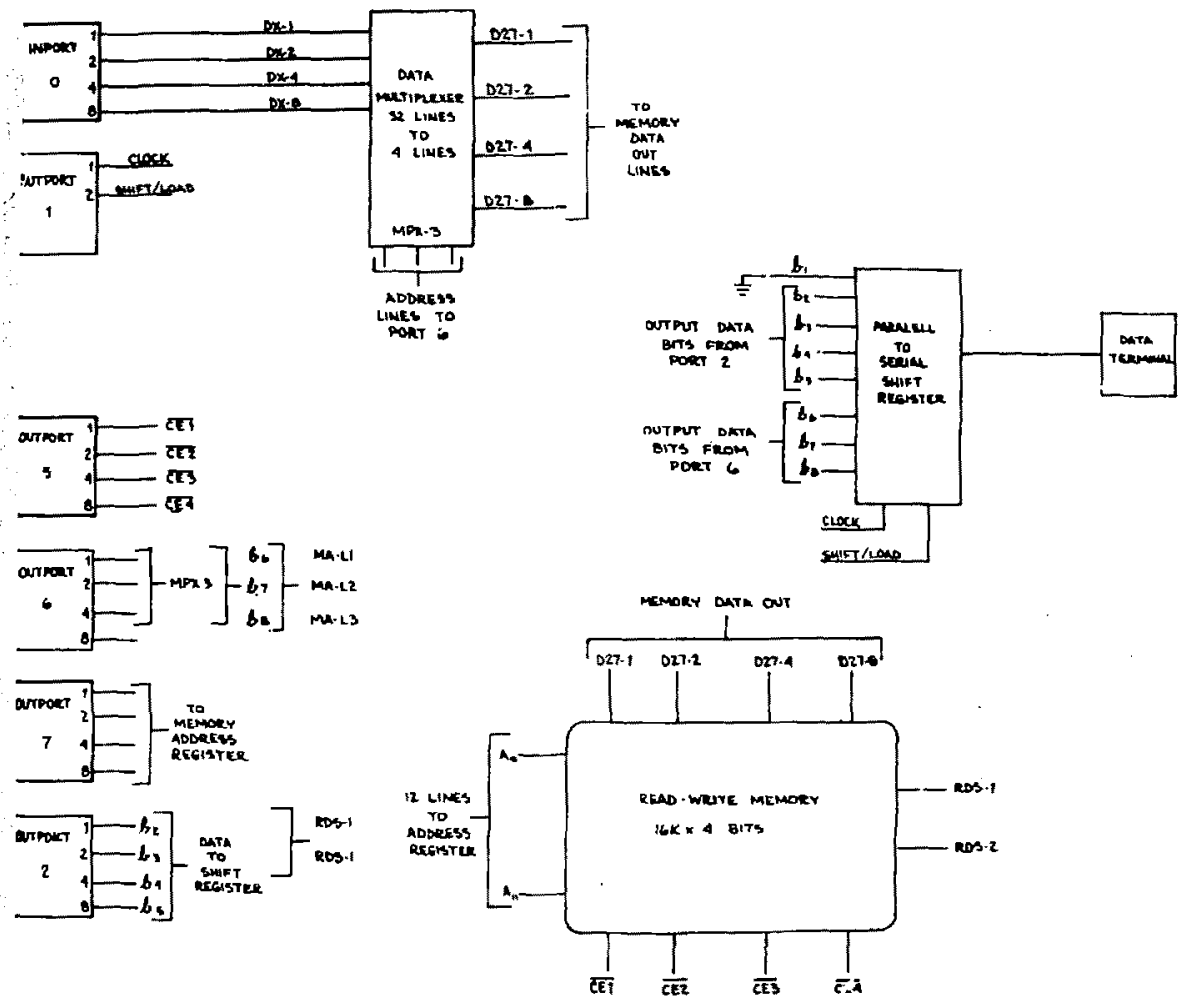

Send Memory Data to Terminal 


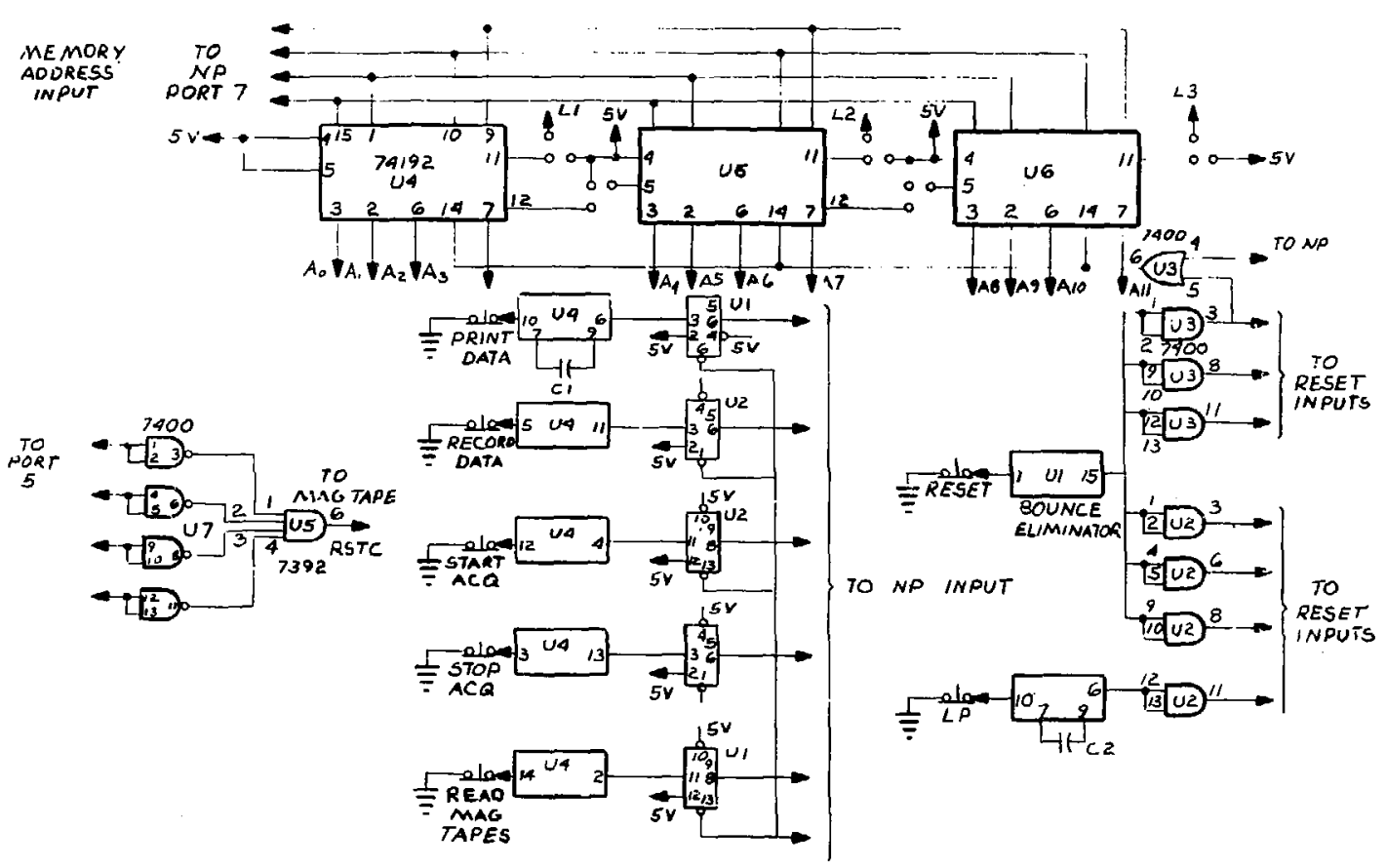

Figure 14. Address Register and Switch Circuits 


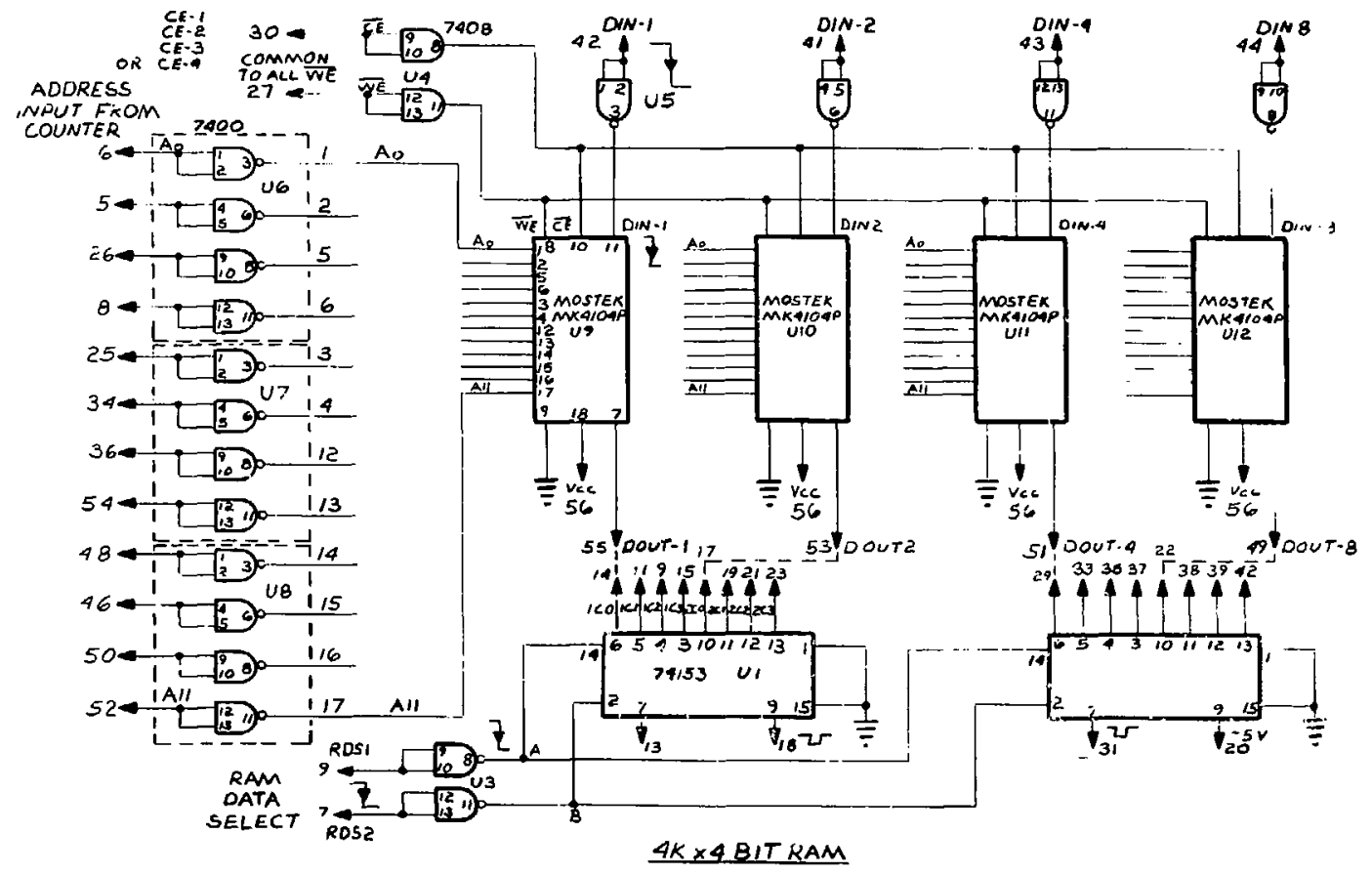

Figure 15. RAM Data Select 
After all addressing is completed, the program pulses the $\overline{C E}$ ine, placing data on the D27 lines, and then reads inport 0 into the CPU accumulator. If no mathematical operation is to be performed, the data character is placed on the broadside inputs of the parallel-to-serial shift register through outports 2 and 6 . The shift/load line is activated; then the clock to outport 1 begins to shift the character to the terminal at a 300 CPS baud rate.

\section{Write Memrry Data to Magnetic Tape}

The procedure previously described for sending data to the terminal is identical for this mode of operation, except that now the magnetic tape unit receives the memory data for storage. Refer to Figure 16 . Data to be stored are placed on lines DB-1 through DB- 8 from outports 2 and 5 . When the read/ write clock (RWCL) signal from the tape unit is received through MPX-3 and inport 0 , indicating that the tape unit is ready to receive a character, signal WXFR from outport 7 first writes the character onto the tape, signal WSTC (writestart) then otarts the tape unit, and finally WSPC (write-stop) stops it when data transfer is complete.

\section{Read Input Characters From Data Terminal}

In this mode of operation the program enters a wait state until a character is received from the terminal. Refer to Figure 17 . When the operator strikes a key, the terminal begine to transmit an 11-bit ASC 11 coded character word at 300 cps baud rate to the systern circuitry (1 start bit, B data bits, 2 stop bits). The first bit (start bit) is detected by the microprocessor which responds by issuing clock pulseg from output 1 to shift the character word into the serjel$\hat{0}$-parallel shift register. After shifting is complete, lines $b-2$ through $b-8$ contain the ASCII coded character; b-1 is ignored since it is the start bit and not part of the coded character. The ASCII coded character is contained on data lines D20 and D21 wired to data multiplexer 2. When MPX-2 and MPX-3 are addressed by the program through outports 6 and 7, D20 and D21 (which represent the alpha-numeric character) are passed to inport 0 and fetched by the microprocessor. The program then branches to perform some action, or if necessary, enter another wait state for the next start bit.

Figure 18 is a detailed sahematic of the serial-to-parallel shift register (74164) which receives the ASCII code alpha-numeric character from the data terminal through the LM1489A bipolar to unipolar convertor. The start bit (bit 1) is detected by the program through UB connected to inport 4 . The parallel-to-serial shift register (74166A) receives a character from outports 4 and 5 . The shift/load and clock respectively load the character and then shift it to the terminal through the LM $1488 \mathrm{~A}$ converter at $300 \mathrm{cps}$ baud rate.

\section{Read Data From Tape and Reload to Memory}

In this mode of operation the tape unit becomes the source of data. Refer to Figure 19. The microprocessor issues a read-start (RSTC) signal which places 


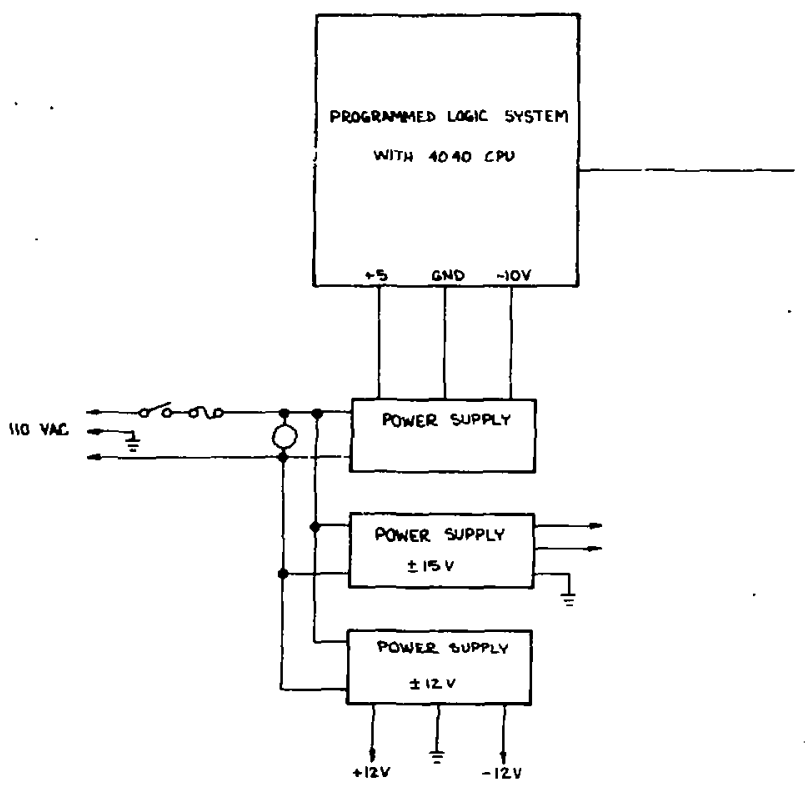

Figure 16. 


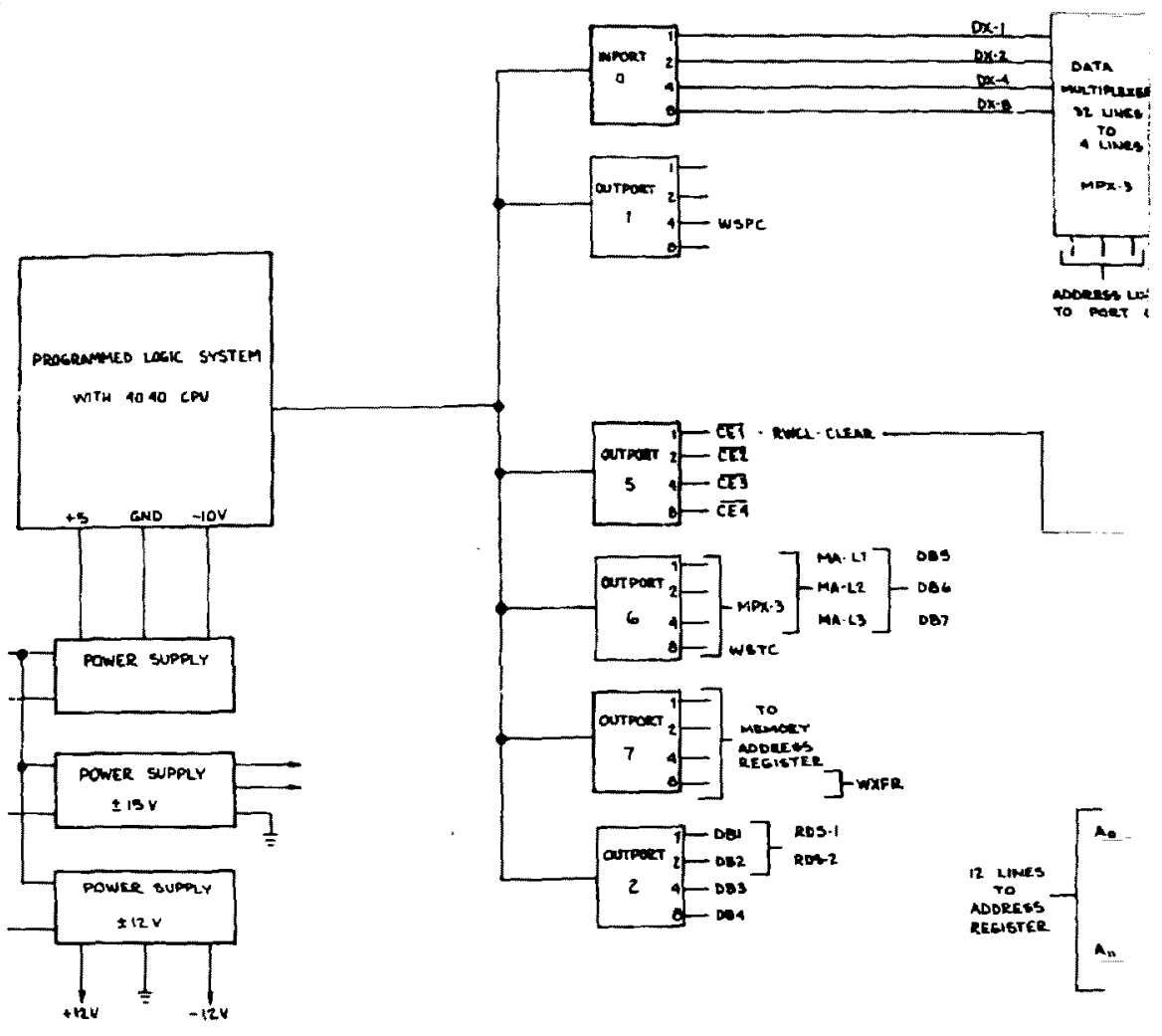

Figure 16. Write Memory Data to Magnetic Tape 


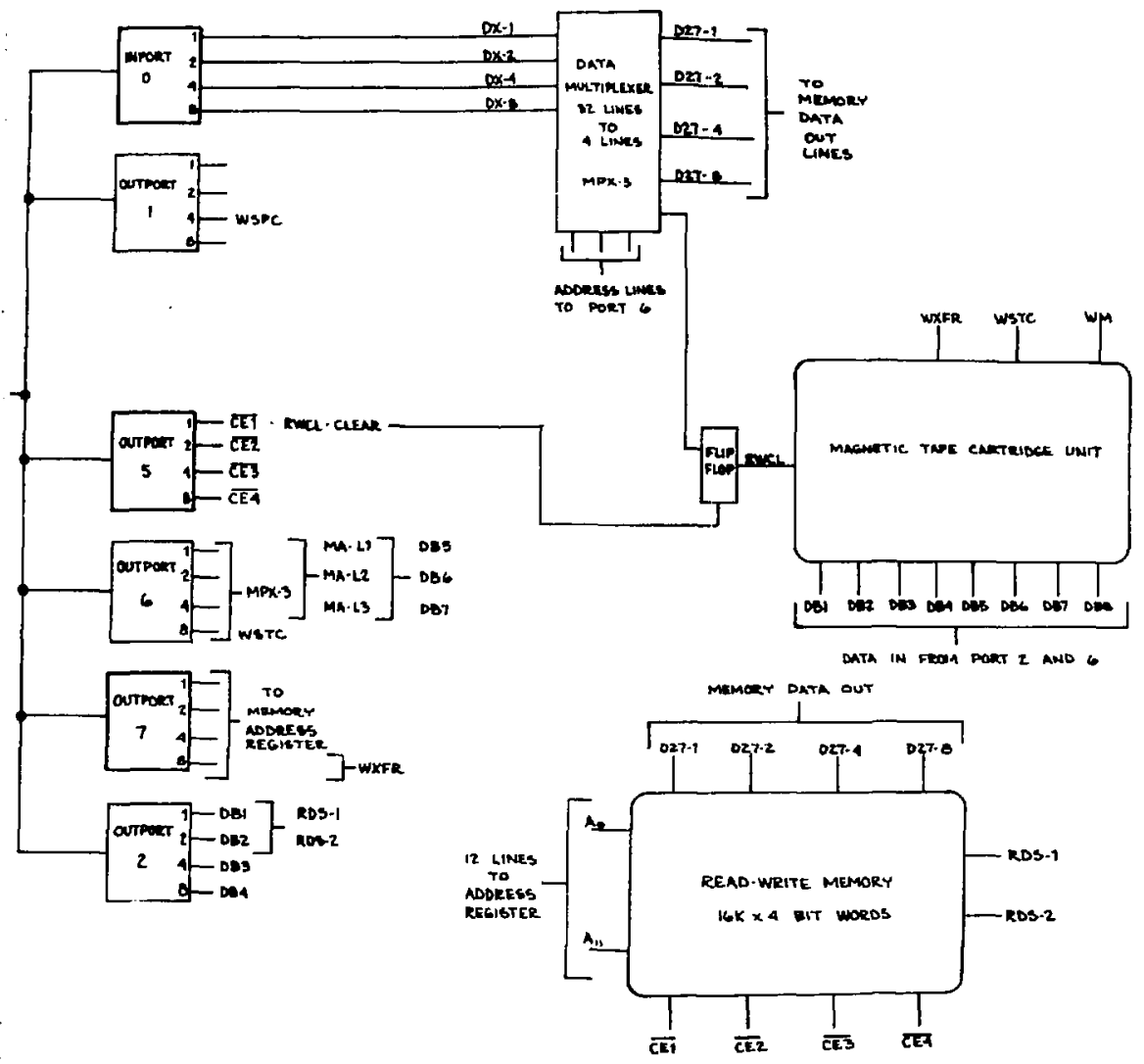

Write Memory Data to Magnetic Tape 


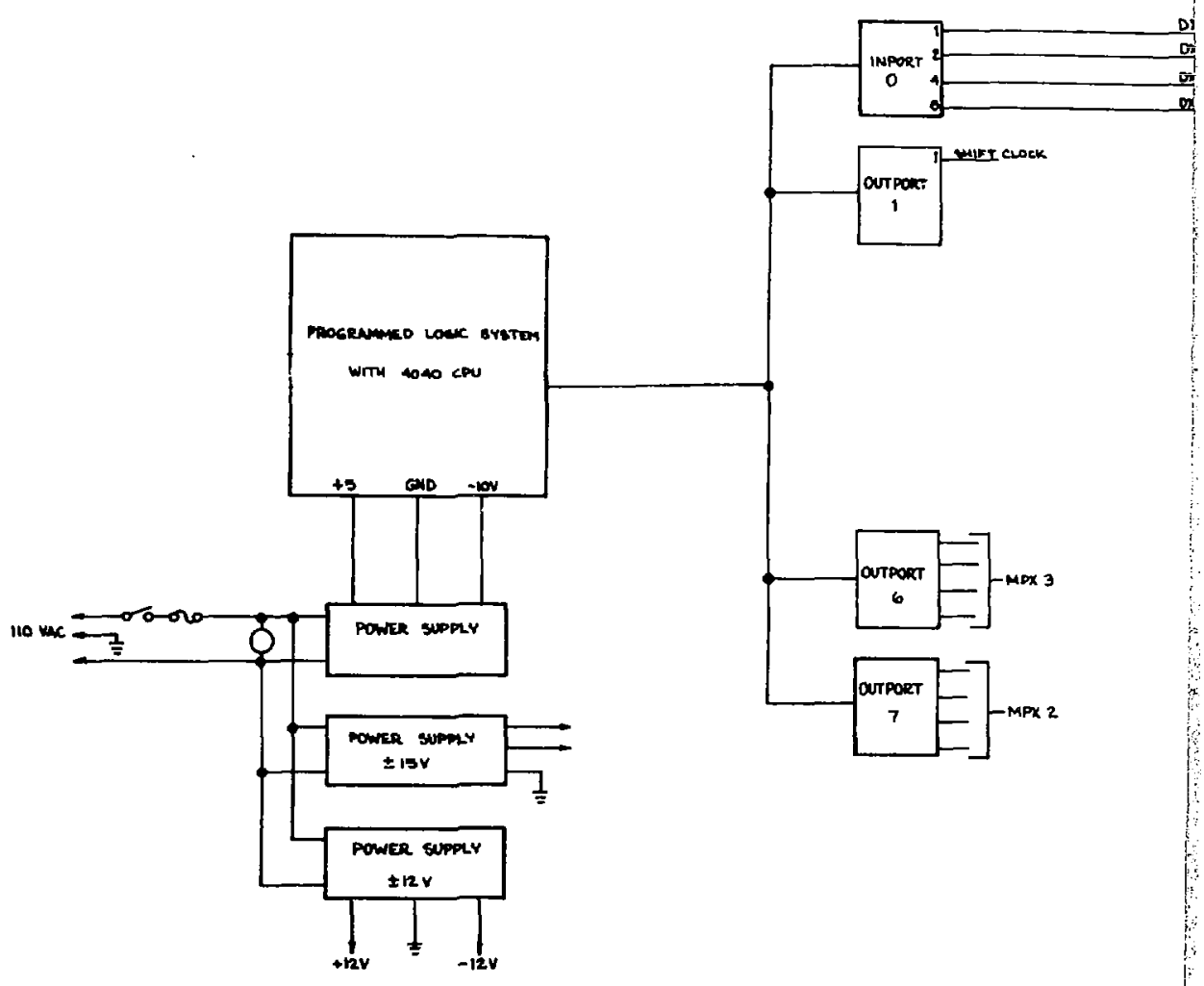

Figure 17. Read Input Characters From Tert 


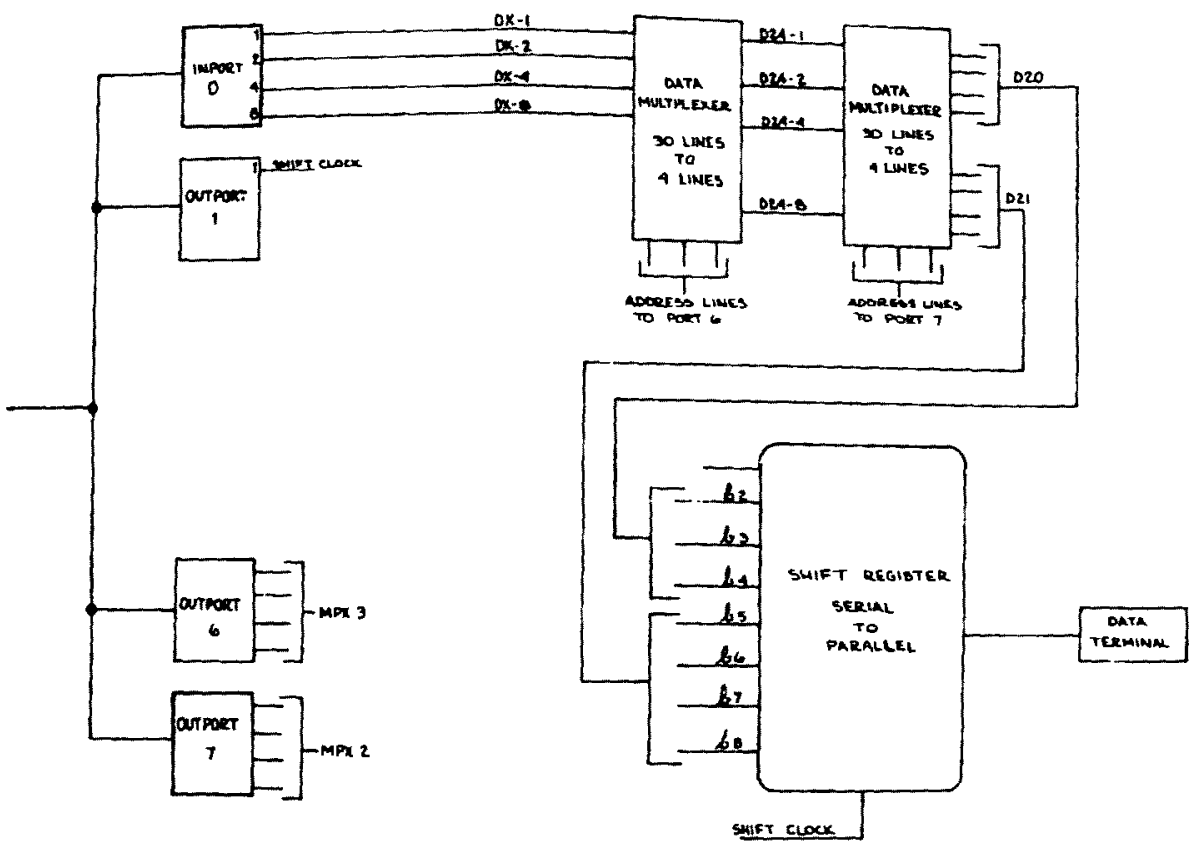

17. Read Input Characters From Terminal 


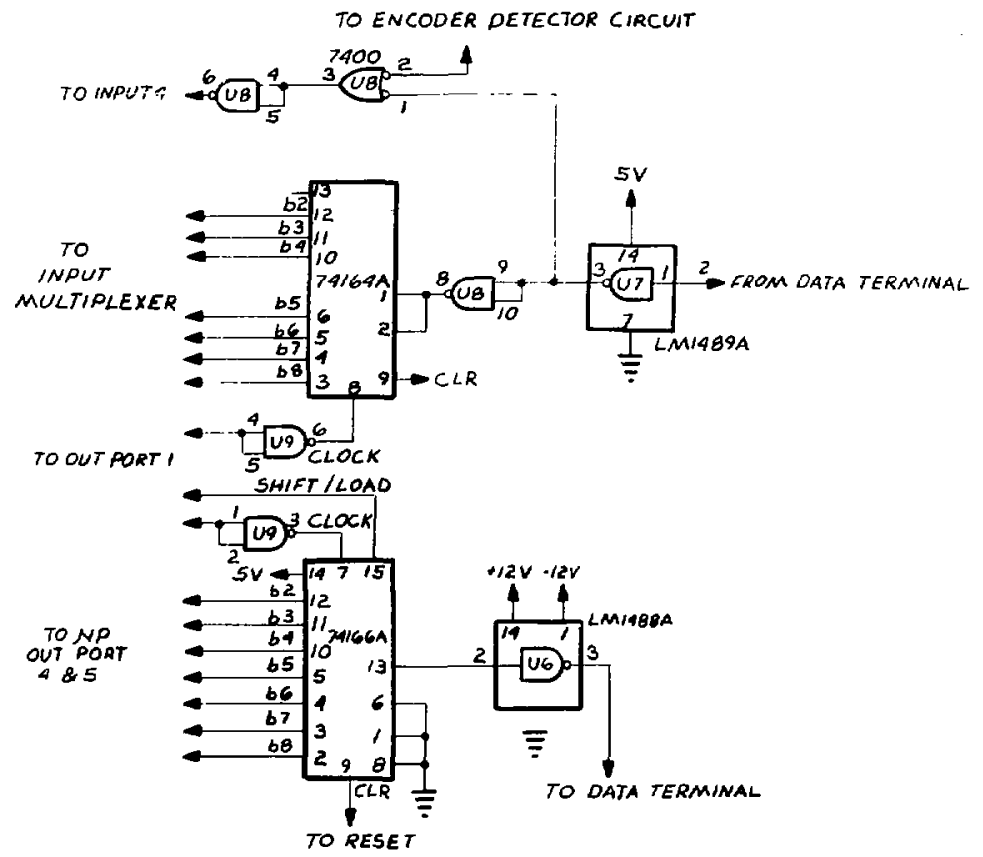

Figure 18. Data Terminal Input and Output Shift Registers 


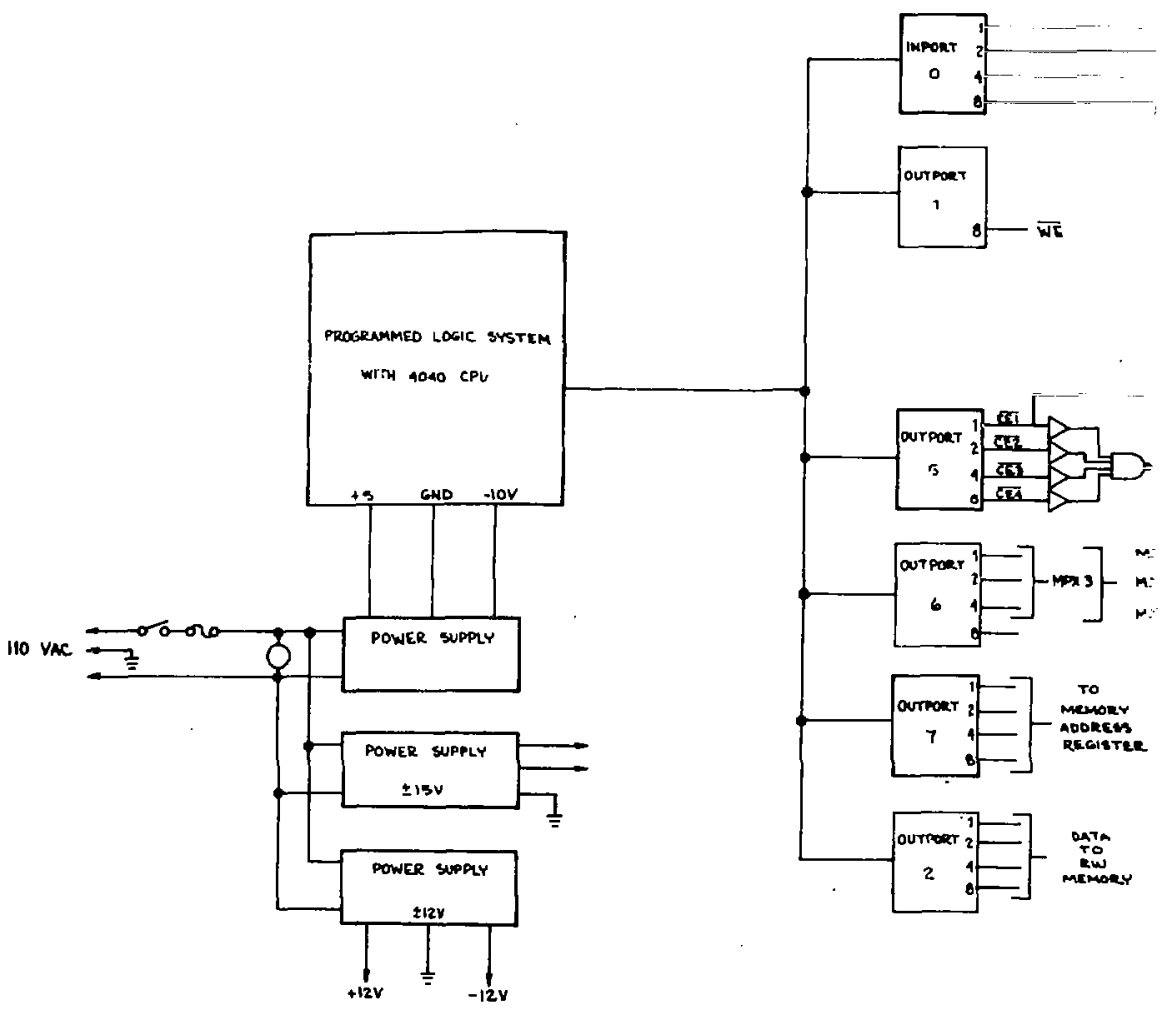

Figure 19. Read Data From Tape and Relo- 


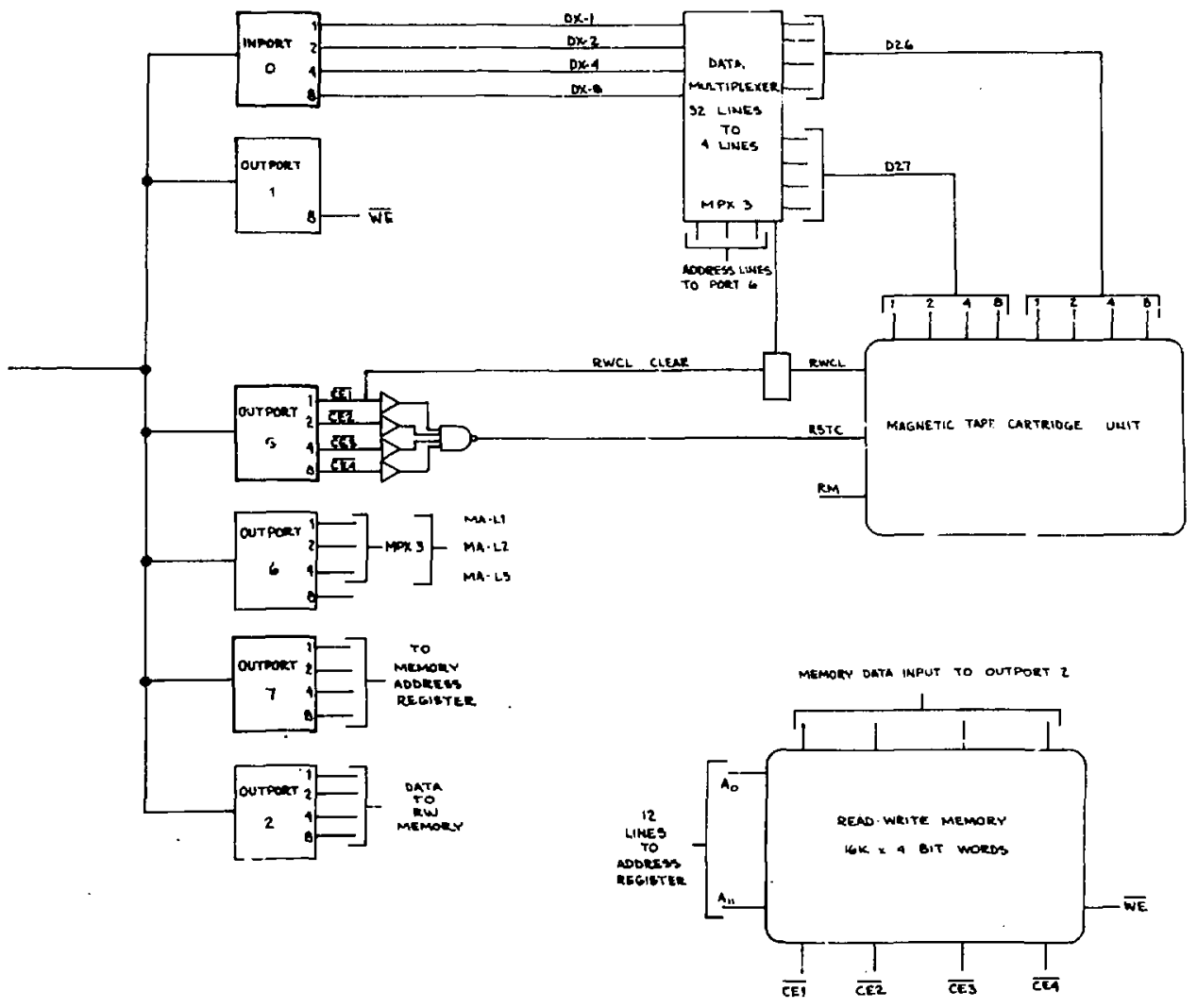

19. Read Data From Tape and Reload to Memory 
the tape unit in the read mode. The tape unit then issues an RWCL indicating that a character is ready to be read. The program, upon detecting the $R W C L$, responds by acquiring the data on lines $D 26$ and $D 27$ by addressing ine MPX-3 multiplexer and inputting the character through ir.port 0 . The program then uperates to write the character in the read/write memory. Addressing takes p'ace as described previously, but now the $\overline{W E}$ (write enable) is actuated before the $\overline{C E}$, thereby effecting a write operation. Tape interrogation and memory writing continue until the full $16 \mathrm{~K}$ locations have been filled.

\section{Data Acquisition and Storage}

The schematic is shown in Figure 20. Upon receiving a nag at inport 4 from the shaft encoder located on the workpiece within the weld chamber, the program issues the Convert signal from outport 1 which causes the analog-todigital converters to convert the analog signal gresent at its input to an equivalent 'igit BCD code ( 12 lines) in 17 microseconds. The progran then operates to acq wre these data and store them in the read/write memory. To achieve this, the 3 cascaded multipl: cers MPS-1, MPX-2, and MPX-3 are addressed to first pass the high-voltage least significant digit (LSD) into inport 0 , store this in memory, and then read and store the second digit of high voltage. This sequence is repeated until all parameters are read and stored in the order shown in Figure 20. Characters are stored by addressing the appropriate locations, then activating the $\overline{C E}$ and $\overline{W E}$ lines as described previously. All input and output ports which are required are ohown in Figure 20. Encoder Ilag detection and parameter storing continues until the memory is full $(16 \mathrm{~K})$ or the Stop switch is pushed by the operator.

\section{Read Memory and Process Data With FPAR}

After parameter date acquisition has been achieved, the operator activates the Print switch, causing the program to ask for a print format code operation ( $A, B$, or $C$ ). If option $A$ is selected the program simply interrogates the memory and passes the characters to the terminal in column form. If $B$ or $C$ format code is selected the operation becomes complex since the measured data are compared with nominal and error-band input values. The results of this comparison determine what is sent to the terminal.

The FPAR (National Semiconductor Model 57109) is used to perform all calculations in making the determinations. Figure 21 is a schematic of the FPAR and peripheral control circuitry.

Functional instructions to the PPAR are through the 2-bit Op code I-5 and I-6 from outport 3 (see Figure 22). Output characters and the Ready-out (RDYO) and Ready-in (RDYI) lines are directed to the microprocessor to multiplexers 2 and 3 when properly addressed by the program to outports 6 and 7. Data $h_{i}$ it to the FPAR is from outport 2 . 


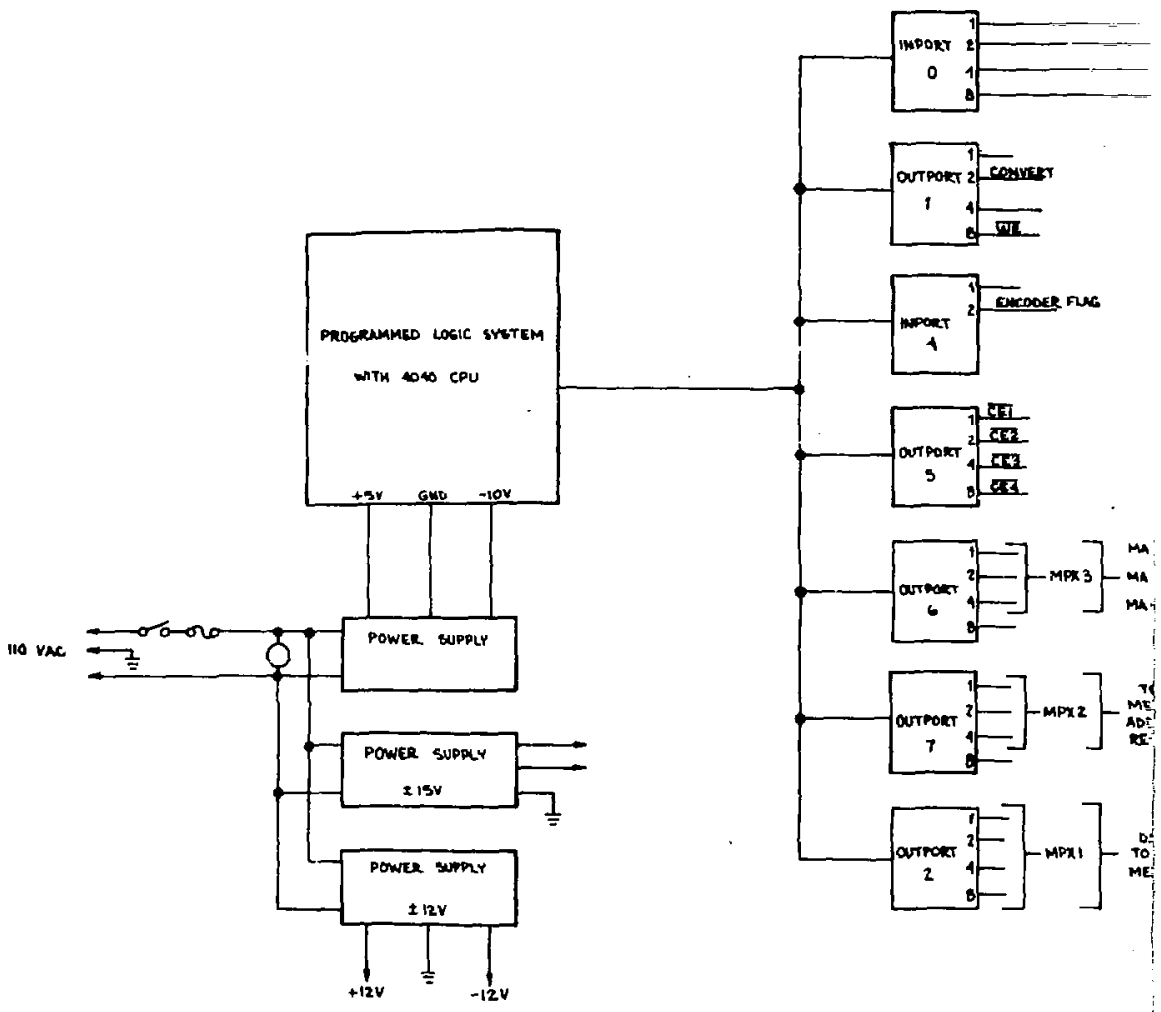

Figure 20. Data Acquisition and Sto 


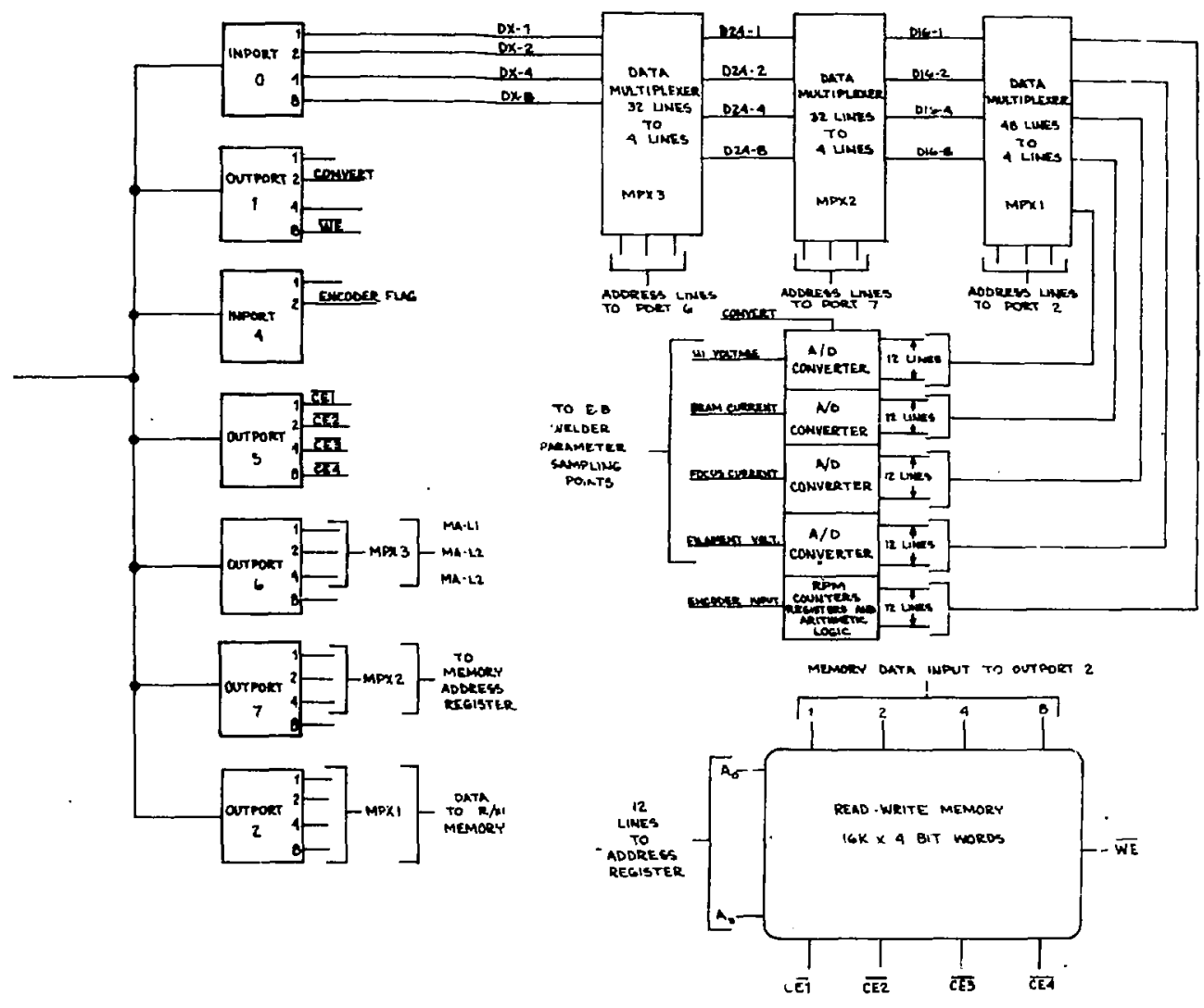

Figure 20. Data Acquisition and Storage 

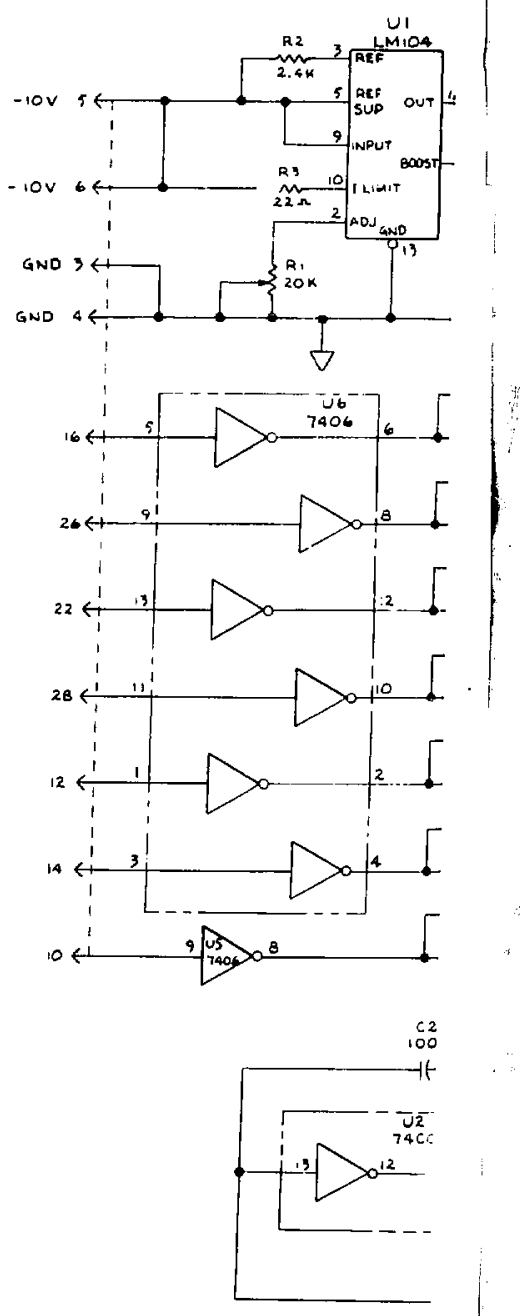


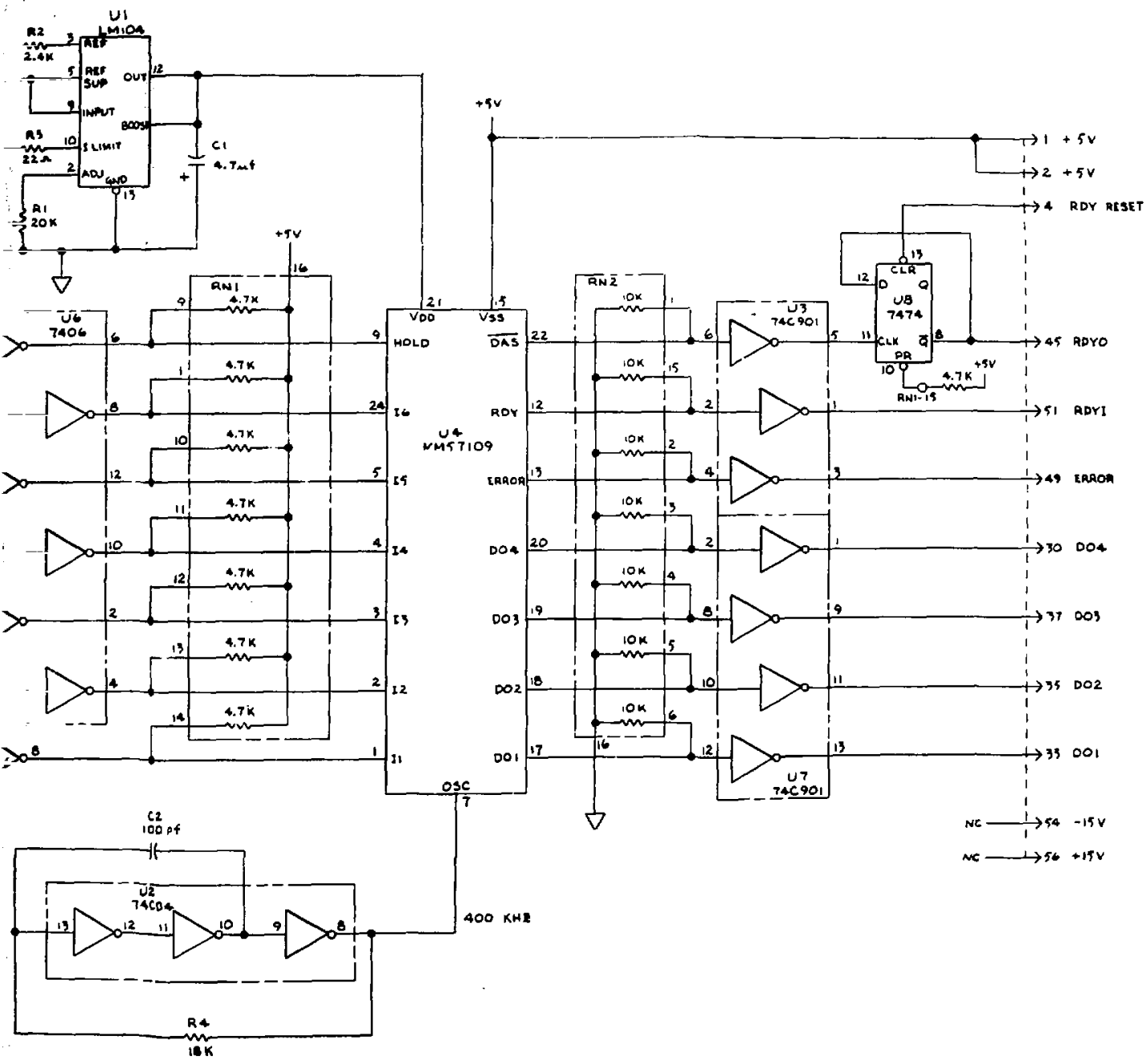

Figure 21. Schematic for FPAR 


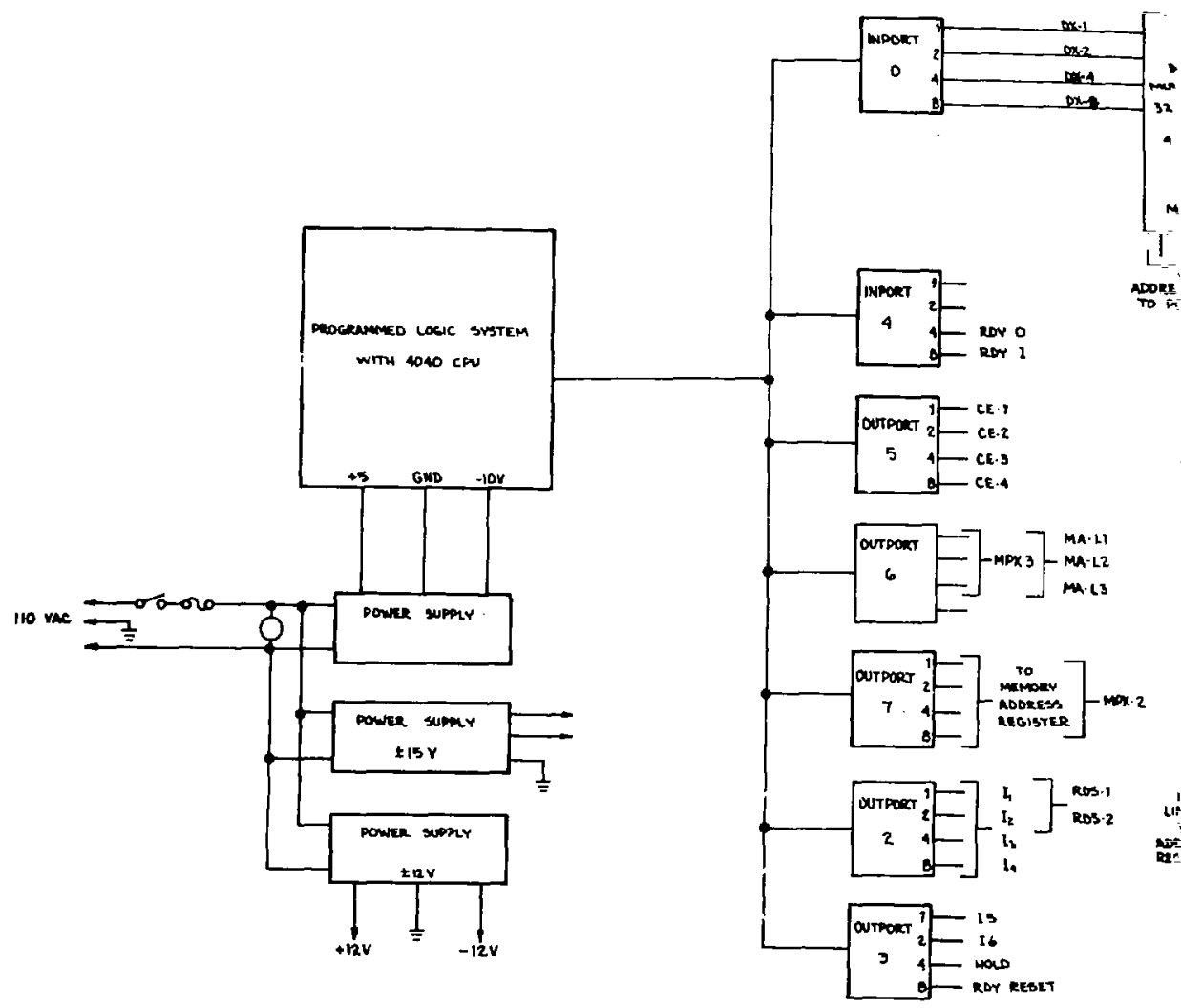

Figure 22. Read Memory and Process Da: 


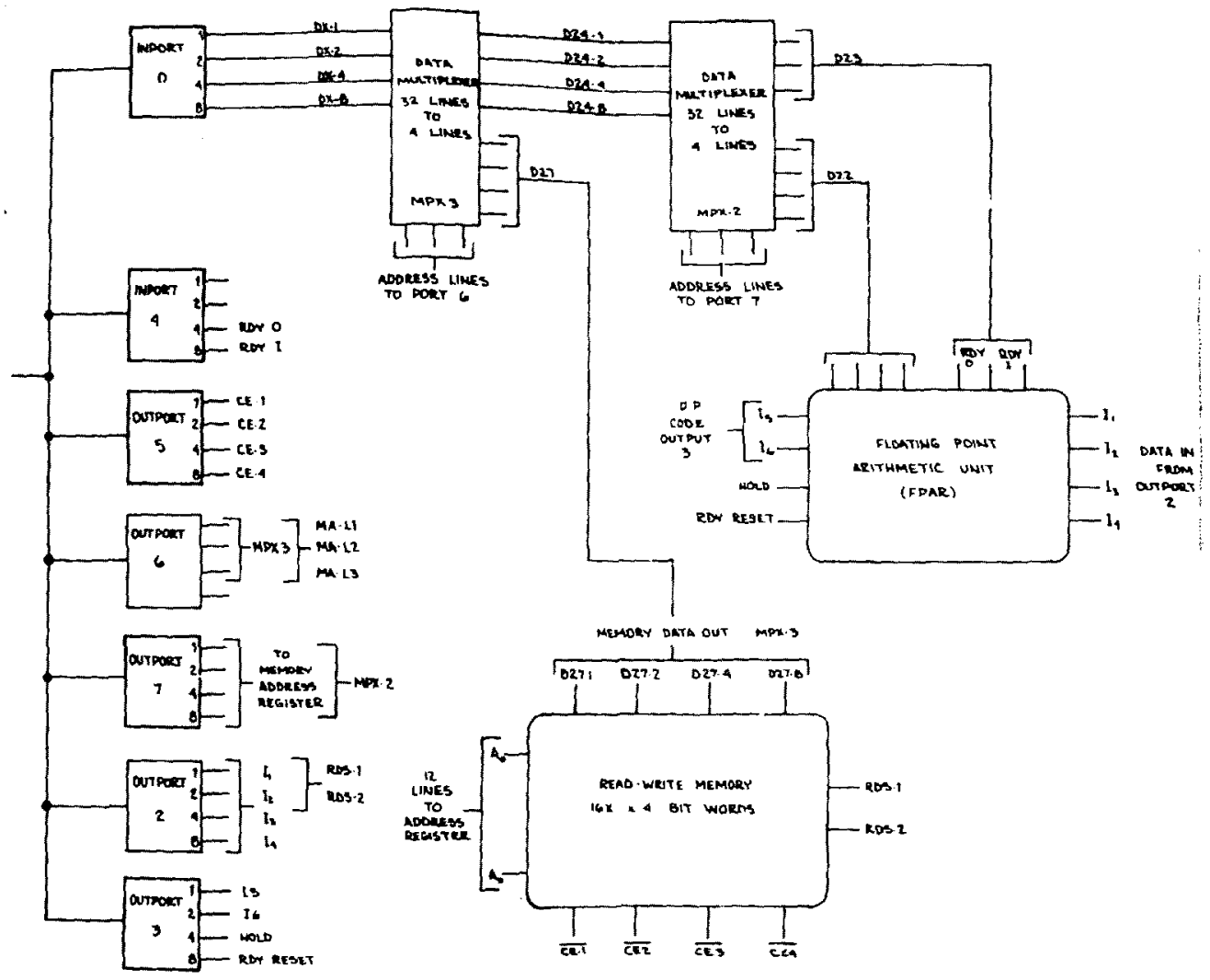

e 22. Read Memory and Process Data With FPAR 
When Reset is activated, the programs enter a subroutine called CALRDY which initializes the PPAR by first manipulating the hold line and waiting for certain timing relationships tetween RDYI and RDY0. A series of 3 commands is then entered: a master clear Op code, followed by an error-clear Op code, and ending with an Op code which has the FPAR accept input in scientific notation. Eight digits, sign, exponent, and exponent sign must be presented to the FPAR in a fixed format (Figure 23a). Flgure 23 is a map of the RAM register located within the programmed logic system. It is used during programming for temporary storage of nominal and error-band input values, FPAR input and output numbers, and other miscellaneous storage. The RAM register 0 is always used as input to the FPAR. Prior to an entry function for arithmetic operation, the 12 digits are first arranged in a correct format under program control. After an operation, the result is removed by the output subroutine and placed in RAM register 1 in the format and location shown in Figure 23b; however, the number in the FPAR is retgined. Because of timing constraints the number is placed as the FPAR offers it in register 1 . Other subroutines then unscramble register 1 contents before moving them to other locations.

Registers 2 and 3 in Figure 23a show the locations where nominal parameter values (entered by the operator prior to the weld) are stored. Values remain until there is a manual reset, they are changed by the operator, or until power is removed. RAM register number 1 (Figure 23b) shows the location of errorband values in register 0 . Register 2 conteins the number of degrees and is incrementeci prior to each line of printout. This is the 3-digit number which is printed Erst.

The programming for the mathematical operation in comparison decisions is shown in detail in the flow chart in Appendix $B$.

\section{Read /Write Memory}

The Read/Write 16K x 4-bit word memory is composed of 4 printed circuit cards, each containing $4 \mathrm{~K} \times 4$-bit words ( 1 page). In addition to the memory, page 1 contains a data multiplexer. The output line from each page is wired to input lines on the multiplexer. In this way the program, through address lines RDS- 1 and RDS-2, selects the page to be interrogated. Gates U6, U7, and U8 interface the memory address register (see Figure 15) and memory address lines A0-A11 which select any one location of the $4 \mathrm{~K}$ of memory. After addressing a memory location, the program can read the memory contents by issuing a $\overrightarrow{\mathrm{CE}}$ (chip enable) signal. The duration of $\overrightarrow{\mathrm{CE}}$ is about 20 microseconds. When the program performs a write operation, data are placed on Din-1 through Din-4, the $\overline{W E}$ (write enable) line is activated, the $\overline{C E}$ line is pulsed, and finally the $\overline{W E}$ is deactivated.

The memory is manufactured by Mostek Corporation with MOS technology. Each unit is packaged in a 16-pin dual in-line configuration and contains $4 \mathrm{~K} x$ 1bit capacity. Four such units are mounted on one page to effect the $4 K x$-bit storage capacity. 


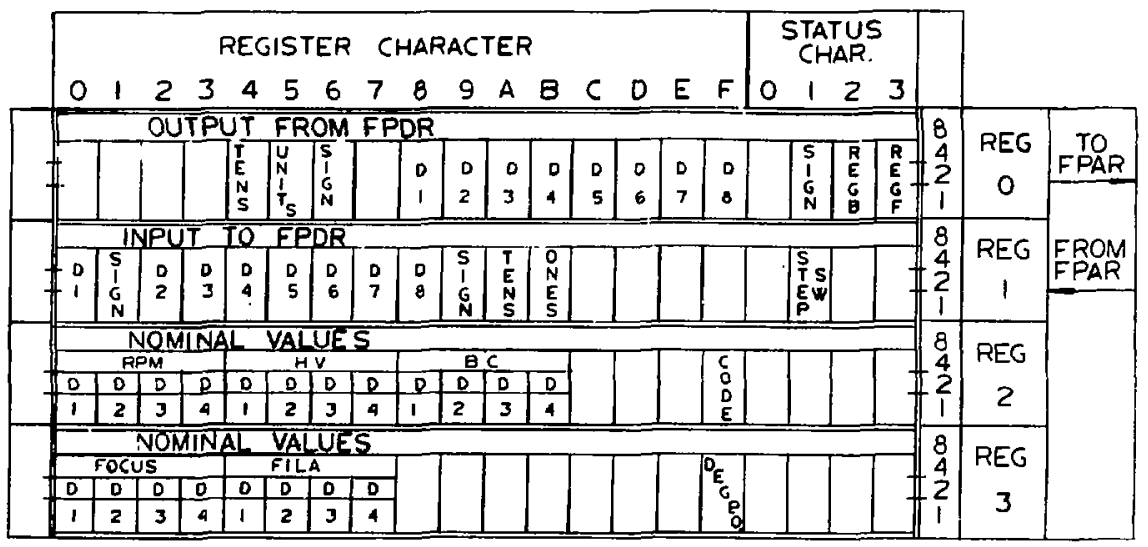

Figure 23a. Intel 4002-1 RAM Register Map \#0

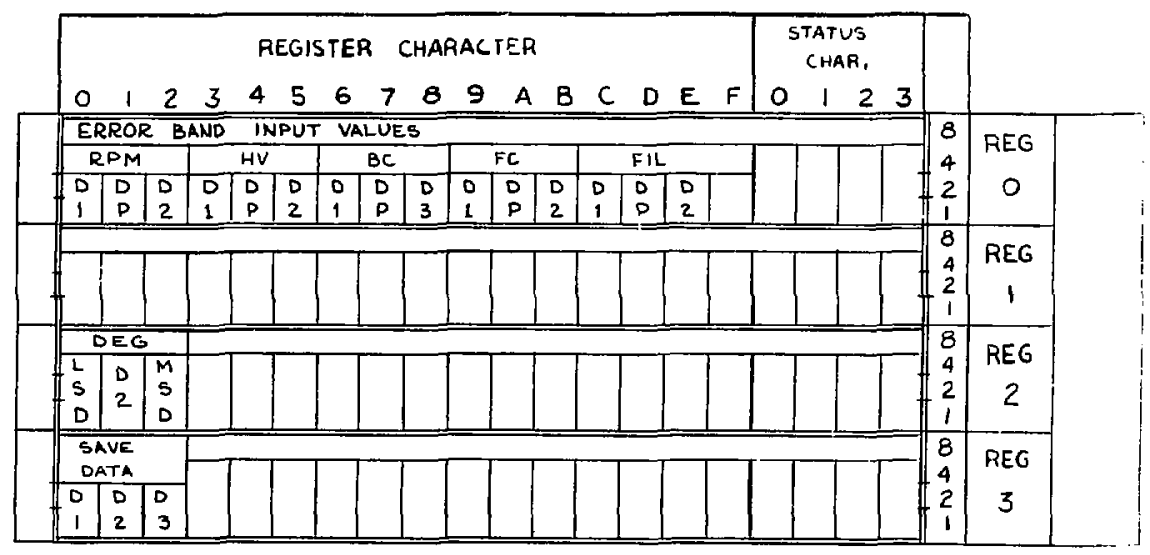

Figure 23b. Intel 4002-1 RAM Register Map \#1 


\section{Address Register and Switch Circuit}

Blocks U-4, U-5, and U-6 in Figure 14 are 3 each, 4-bit binary storage registers used for addressing the read/write memory. The address is placed in the register by the microprocessor by first placing the address at outport 7 lines and then activaring the appropriate load line L1, L2, or L3 through outport 6. Thus the program has the capability of randomly selecting any location within memory.

All front-panel switches are connected to de-bouncing circuits and then to a flip-flop. The $Q$ outputs from the flip-flops are wired to multiplexers, which eventually enter the microprocessor where their active state is detected and appropriate action is taken by the program to perform the commanded function.

\section{Magnetic Tape-Unit Data and Control Circuit}

Data to be written onto magnetic tape are first placed at lines DBT-1 through DBT-4 from the microprocessor outport 3 (see Figure 24). Since only numeric characters are stored, more than 4 lines are unnecessary. Therefore, DBT-5 through DBT-8 are wired to a permanent logic 0. Data lines from the tape unit are used for both input and output, depending on the status of RM (read mode) and WM (write mode) signal lines. These two lines are used to place the 4126 gates in either a high $\mathrm{Z}$ (open switch) state or low $\mathrm{Z}$ (closed switch) state. For example, when the tape unit is in the write mode the WM lines go to $0 \mathrm{~V}$, effectively opening circuit $\mathrm{U}-1$. $\mathrm{RM}$ is at $5 \mathrm{~V}$, allowing data to pass through gate $\mathrm{U} 3$ to be written onto tape.

The right side of Figure 24 shows the signals and corresponding connector pin numbers which pass to and from the magnetic tape unit and microprocessor. The read-write clock (RWC1) signal is applied to a flip-flop because its duration is too short to be detected by the microprocessor. The flip-flop stores the RWCL signal until the cmiroprocessor has time to detect its presence at inport 0 , after which the program resets the flip-flop from outport 5 .

\section{Microprocessor Port Assignments}

Figure 25 shows all input and output ports of the programmed logic system. All data and control lines associated with each port are shown. Because of the many modes of operation associated with the complete operation, some ports have multiple functions. For example, outport 2 supplies data to the tape unit, data terminal, and RAM. This is possible because only 1 unit is activated at any time. A unit being addressed receives the data whereas the other cannot since its control lines are deactivated. This scheme permits multiple operation without increasing the number of ports. 

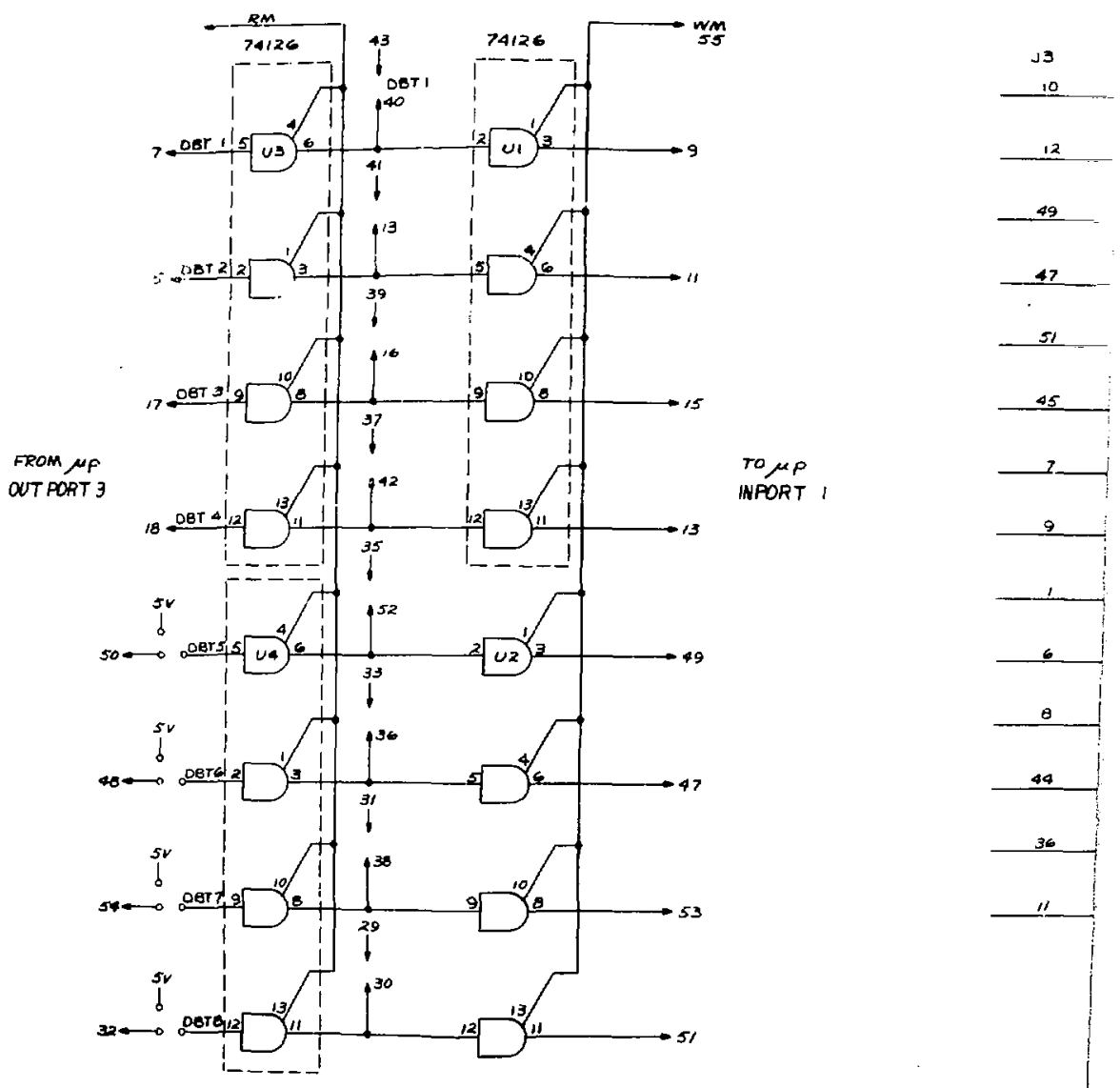

Figure 24. Magnetic Tape Unit Data 


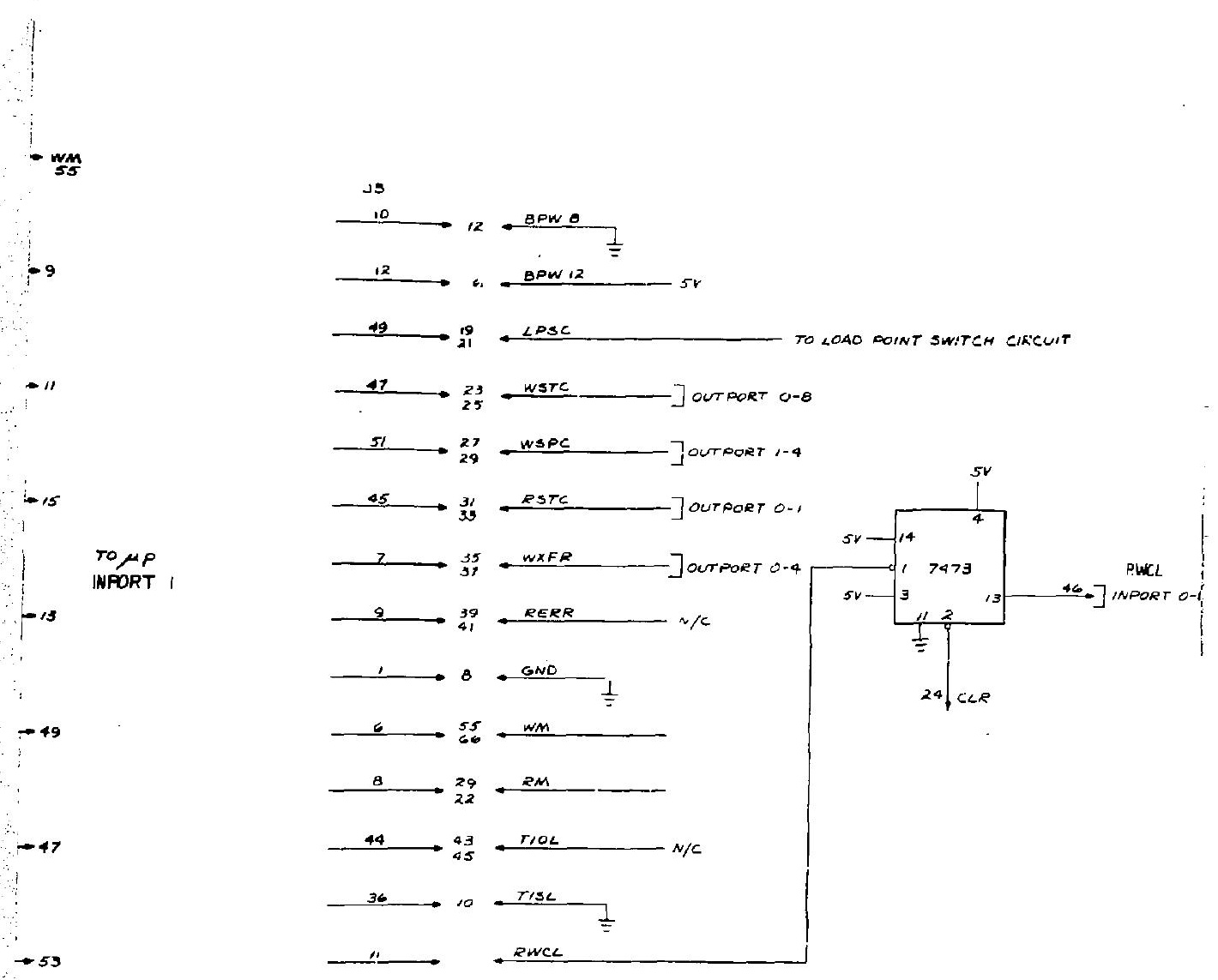




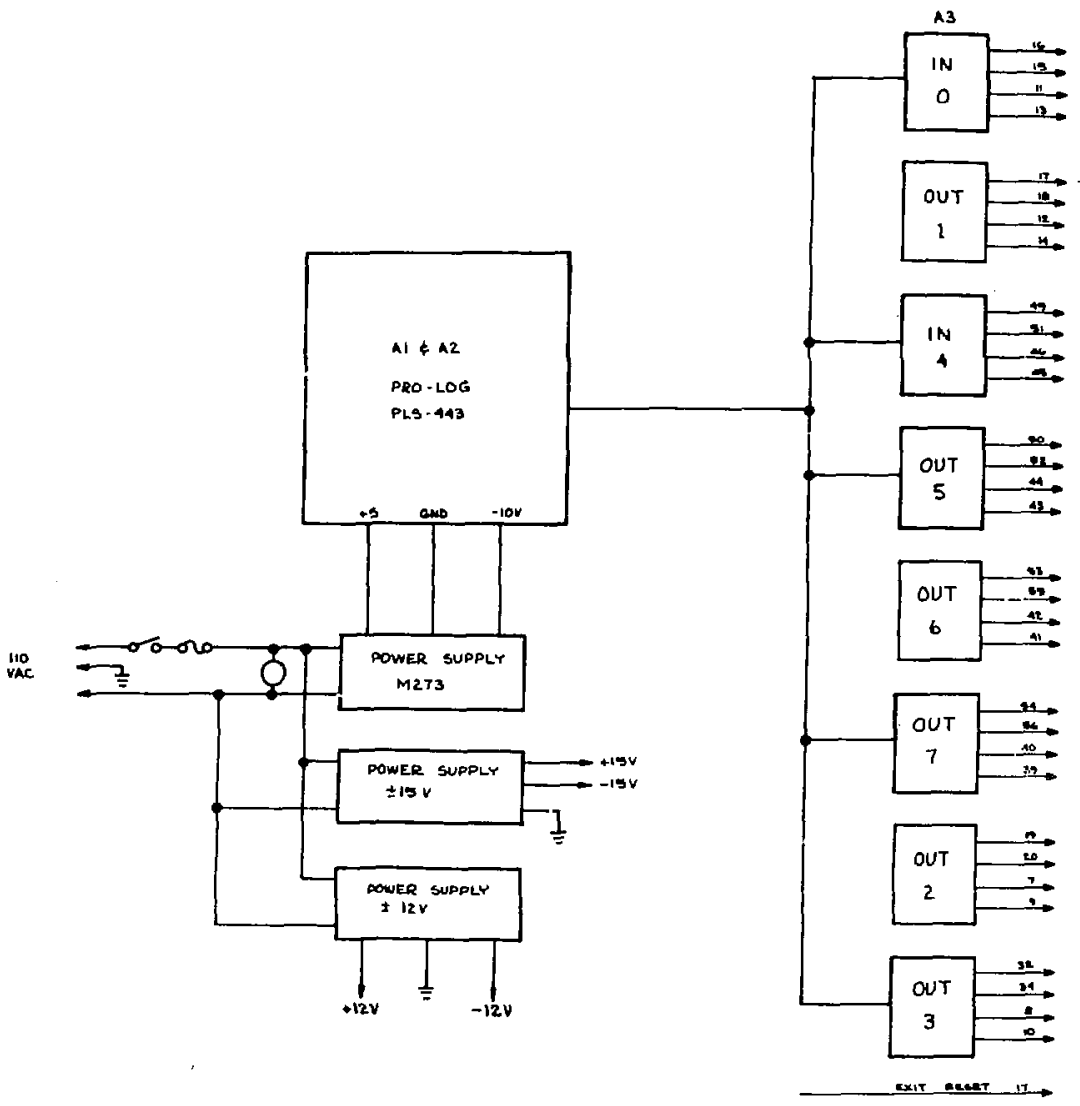

Figure 25. Microprocessor Port Assignme 


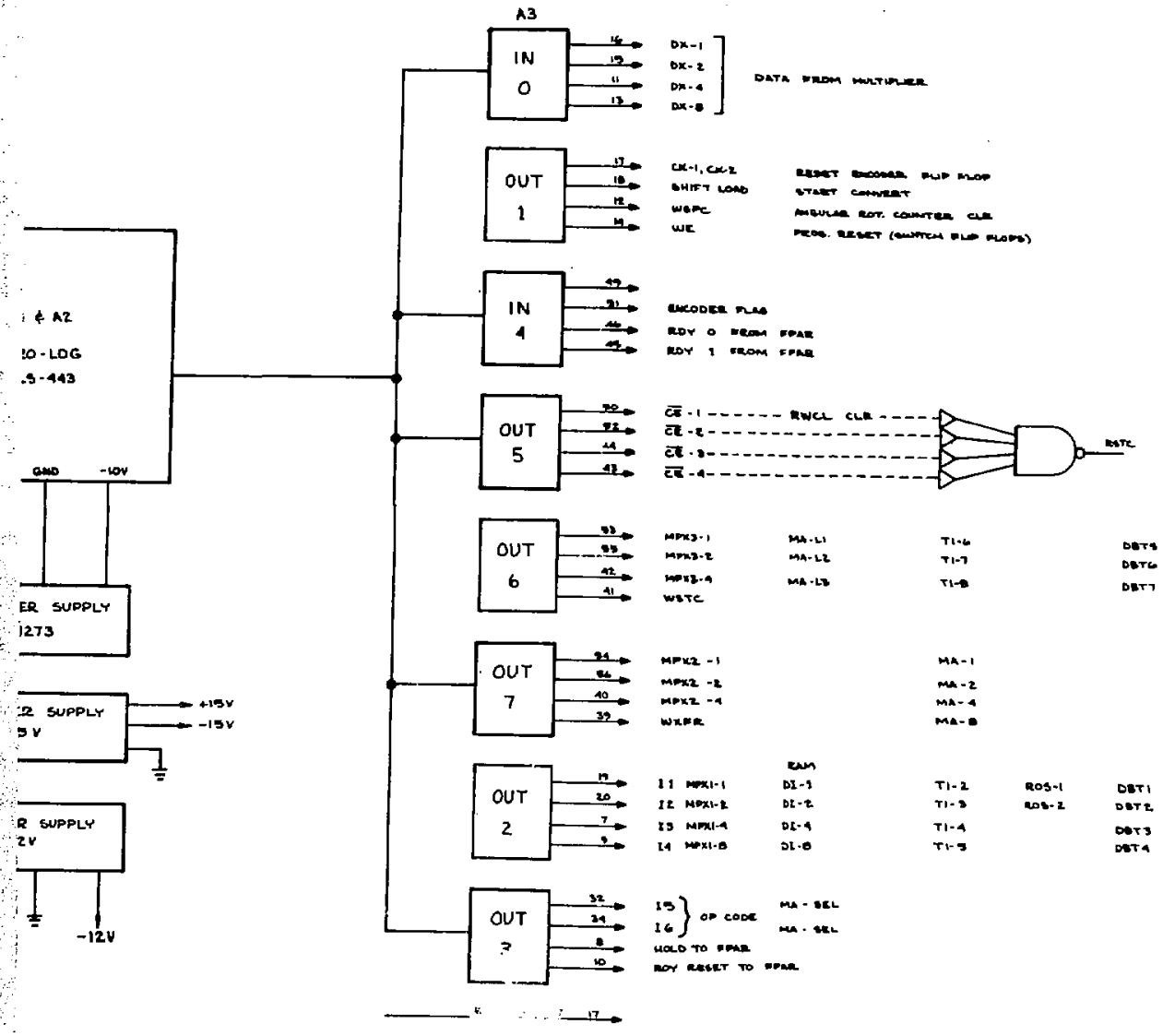

Figure 25. Microprocessor Port Assiguments

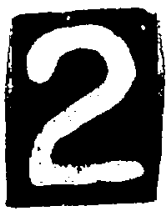


APPENDIX A--MISCELLANEOUS DRAWINGS AND DIAGRAMS 


\section{Calibrator}

A schematic for the calibrator is shown in Figire A-1. This calibrator permits simulation of parameters for a functional test of the data acquisition system. A connector mounted on the rear of the unit receives the cable from the system which normally connects to the welder. On the front panel of the calibrator there is one potentiometer each for the high voltage, beam current, and focus current. To allow a parameter to be set accurately, a three-way selector switch is used to display, on a front panel digital voltmeter, the value being adjusted.

The simulated filament voltage is fixed at $97.0 \mathrm{~V}$. For the workpiece RPM, a crystal-controlled oscillator supplies a simulated encoder signal. The fixed frequency is equivalent to a constant 2.1 RPM.

\section{Encoder}

Figure A-2 is a schematic of the encoder power supply and interface. The encoder, which is attached to the fixture holding the part to be welded, puts out 360 pulses per complete revolution. It is a type $82 \mathrm{H} 360$ manufactured by Dynepar Corporation. Nolse transients generated by high-voltage discharges within the welding chamber can be transmitted to the data acquisition system electronics and damage components. To prevent this, an optical izolator is interposed between the encodur signal output at the input to the system. Also, the encoder is powered by a dedicated power supply. 


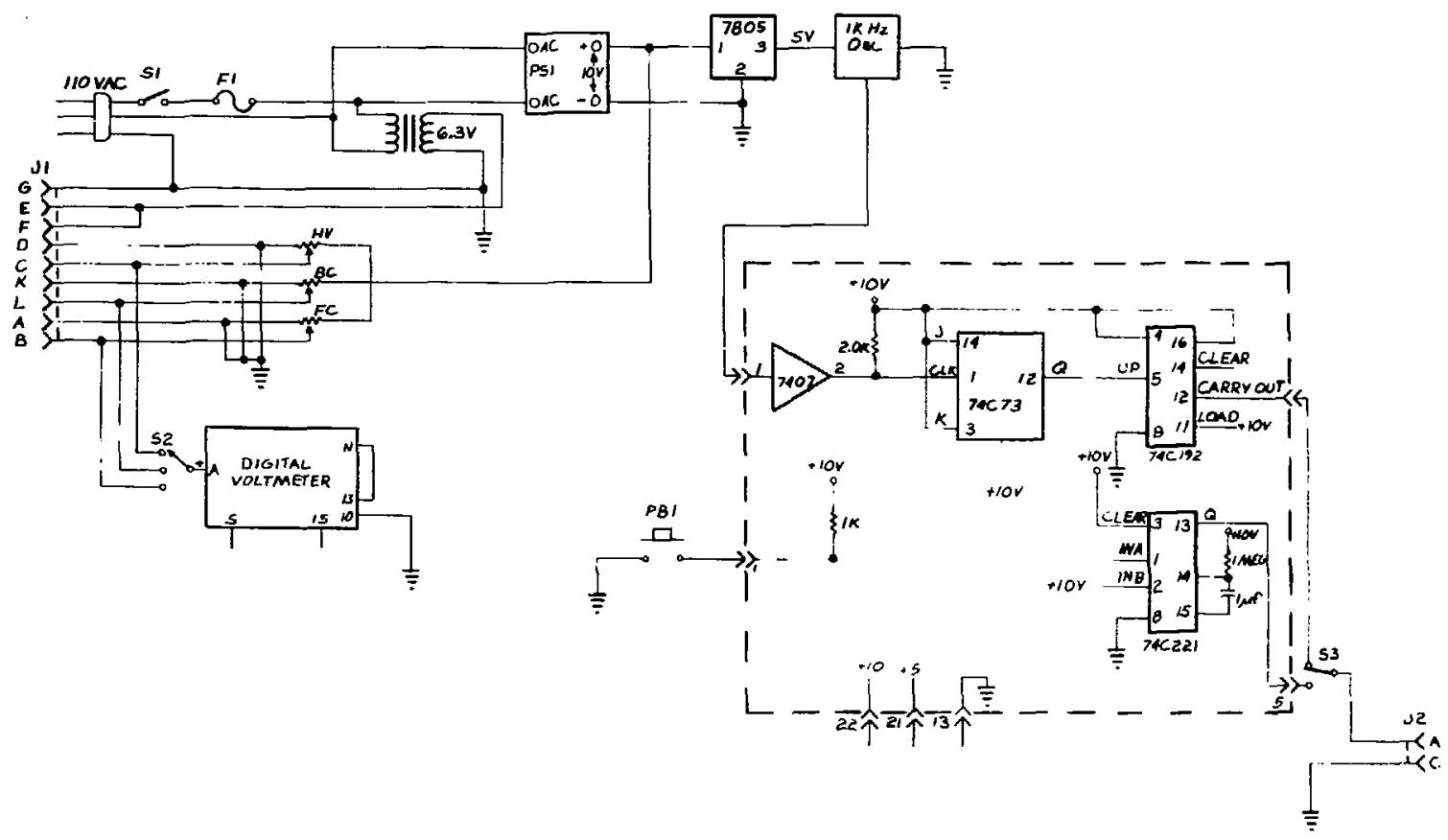

Figure A-1. Data Acquisition Calibrator 


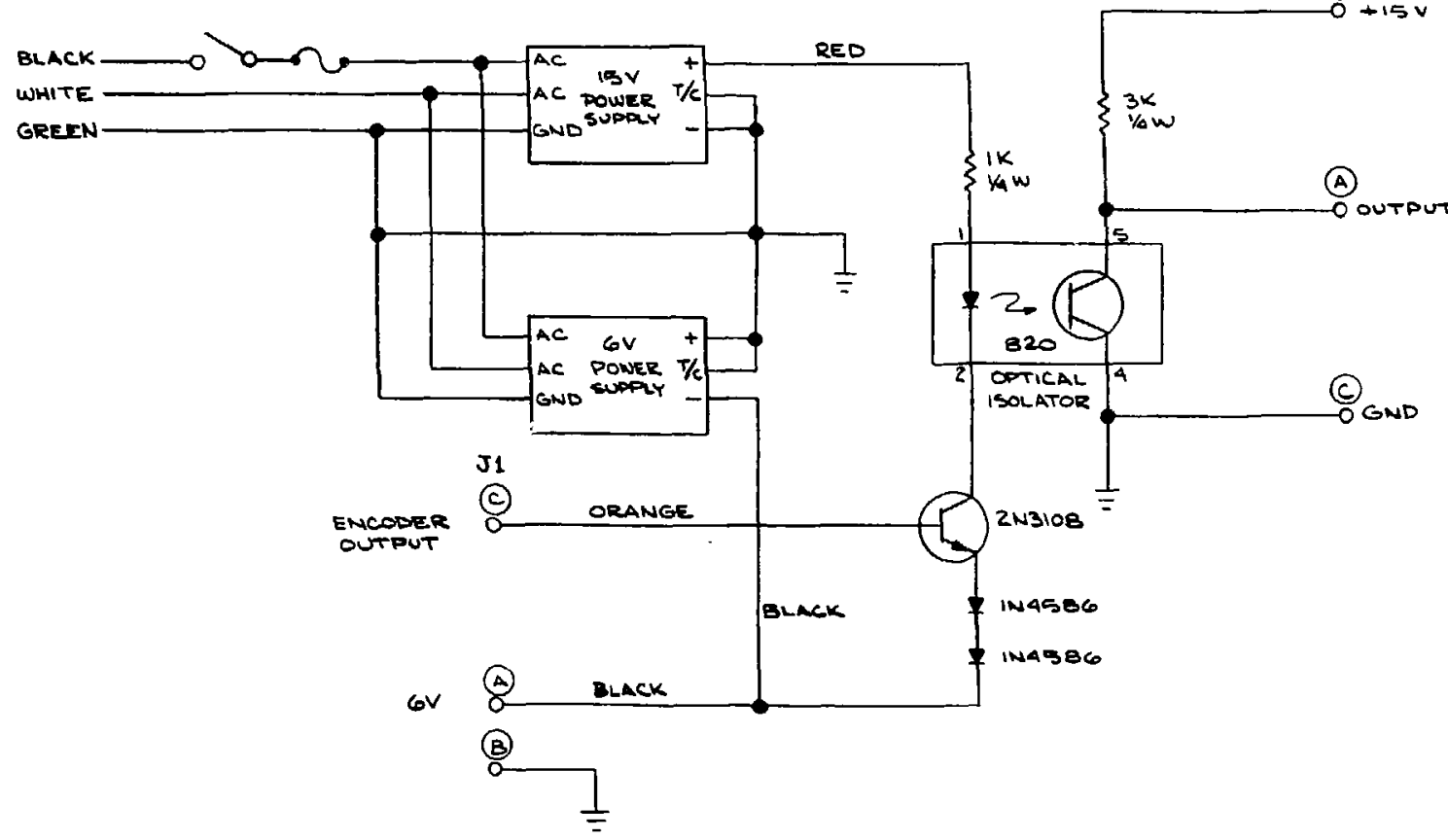




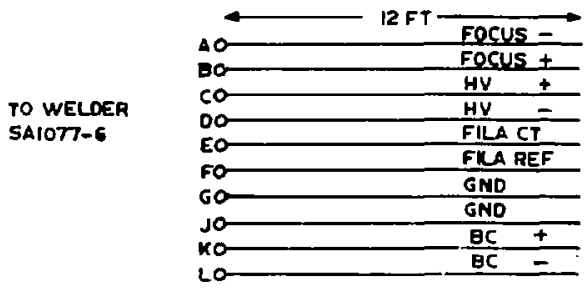

TO CONNECTOR

TERMINAL STRIP

Figure A-3. Diagram of the Cable Between the Connector Mounted on the Welder for Parameter Sompling) and the Terminal Strip Mounted on the Rear of the Data Acquisition System

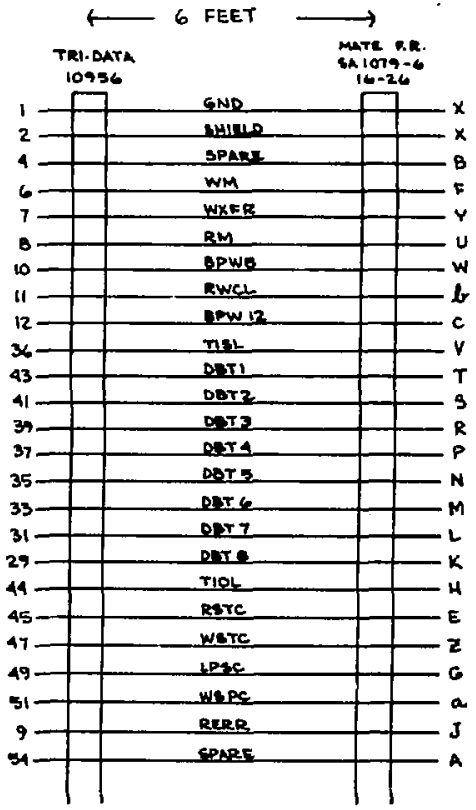

Figure A-4. Wiring Diagram Between Mag Tape Unit and DAS 

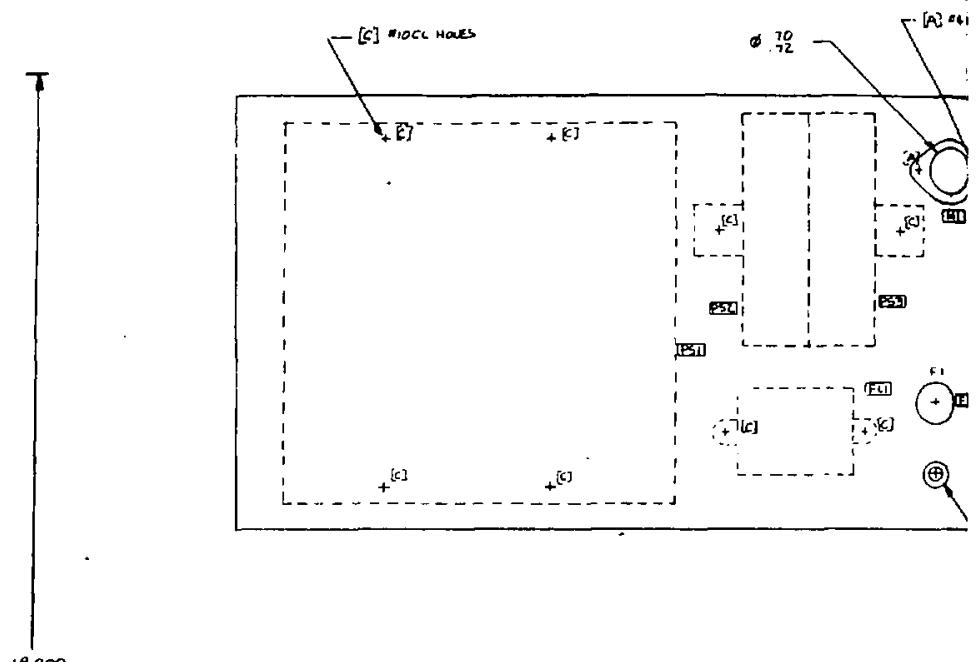

18000

$\mid$

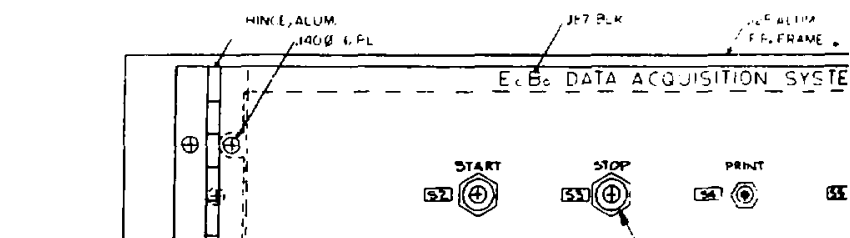

Figure A-5. Mechen Data $d$ 

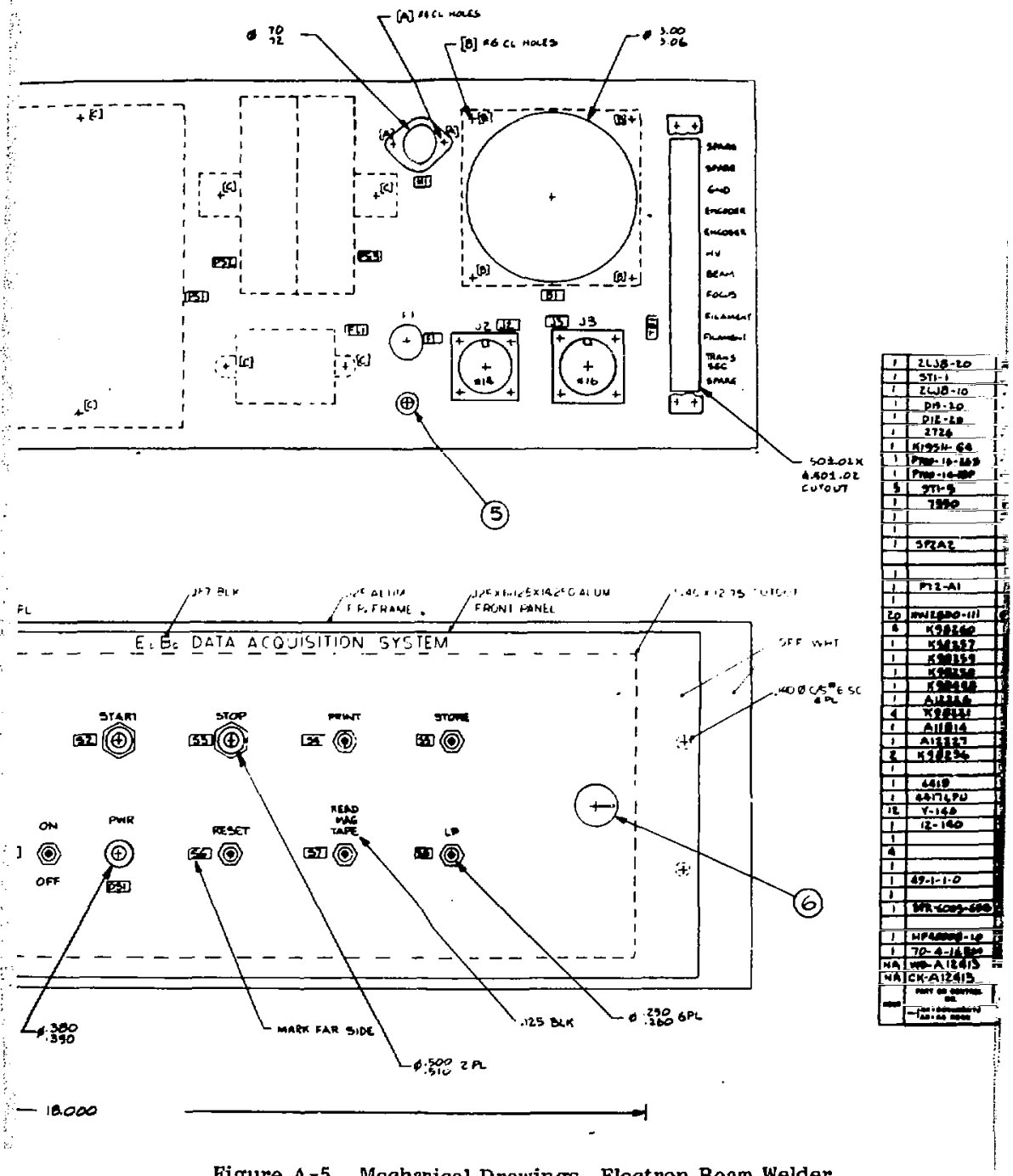

Figure A-5. Mechanical Drawings, Electron Beam Welder Data Acquisition System 
Mec nad

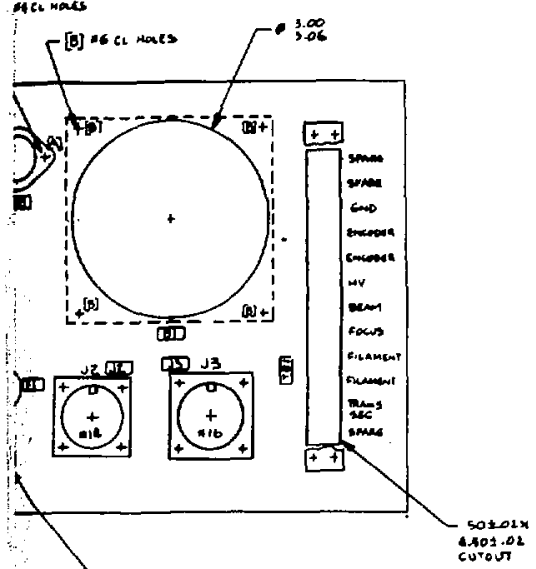

(5)
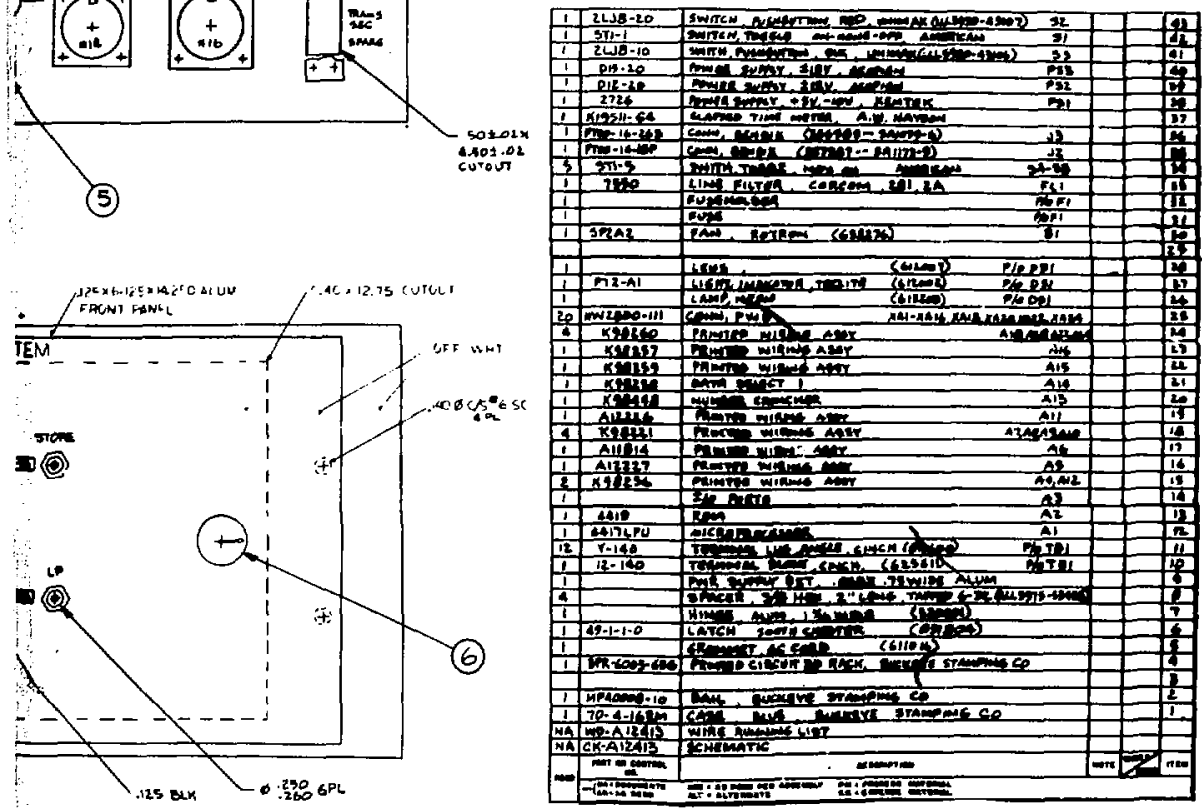

1510 2n

nical Drawings, Electron Beam Welder Acquisition System 


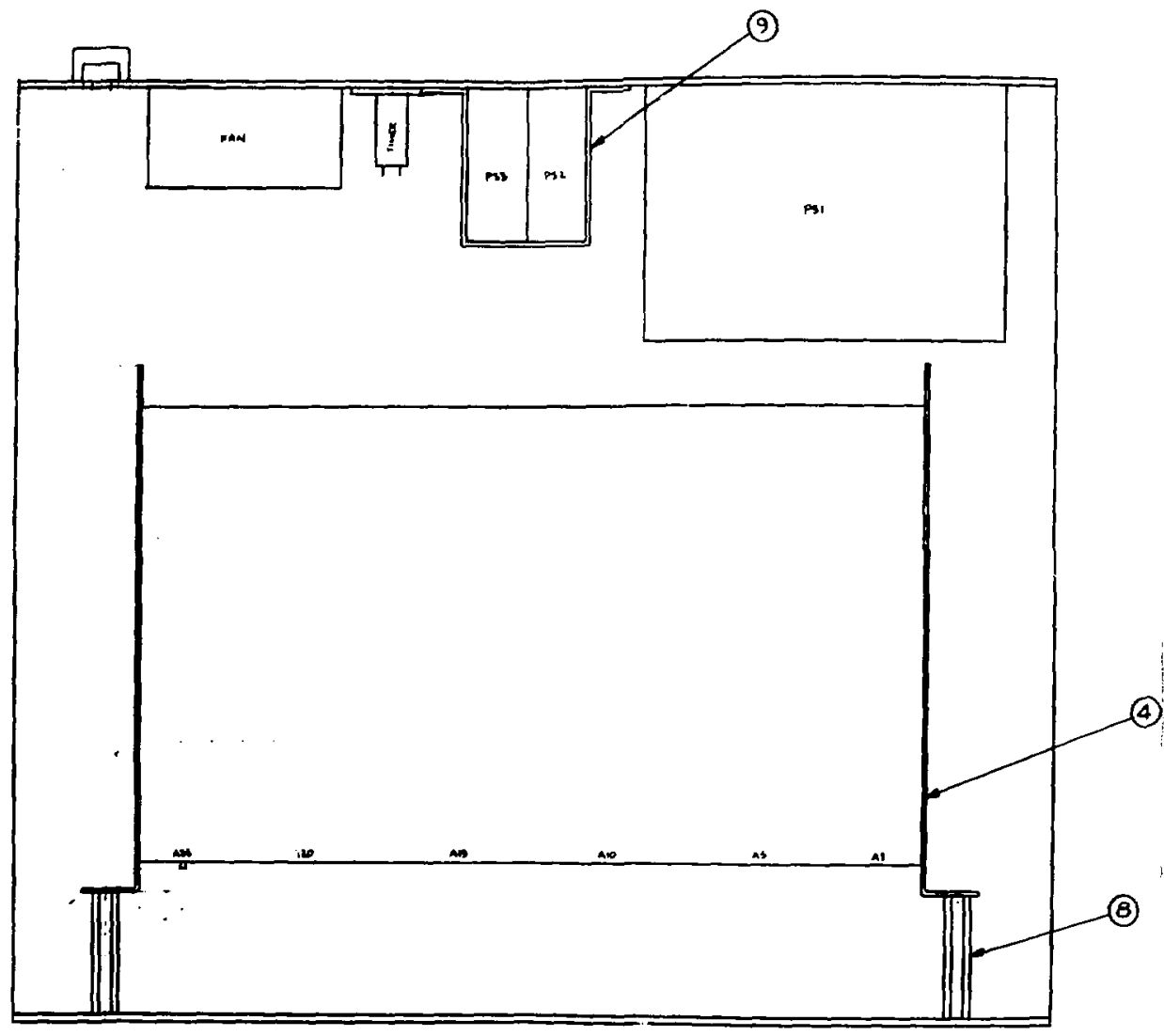

Figure A-5. (continued) 


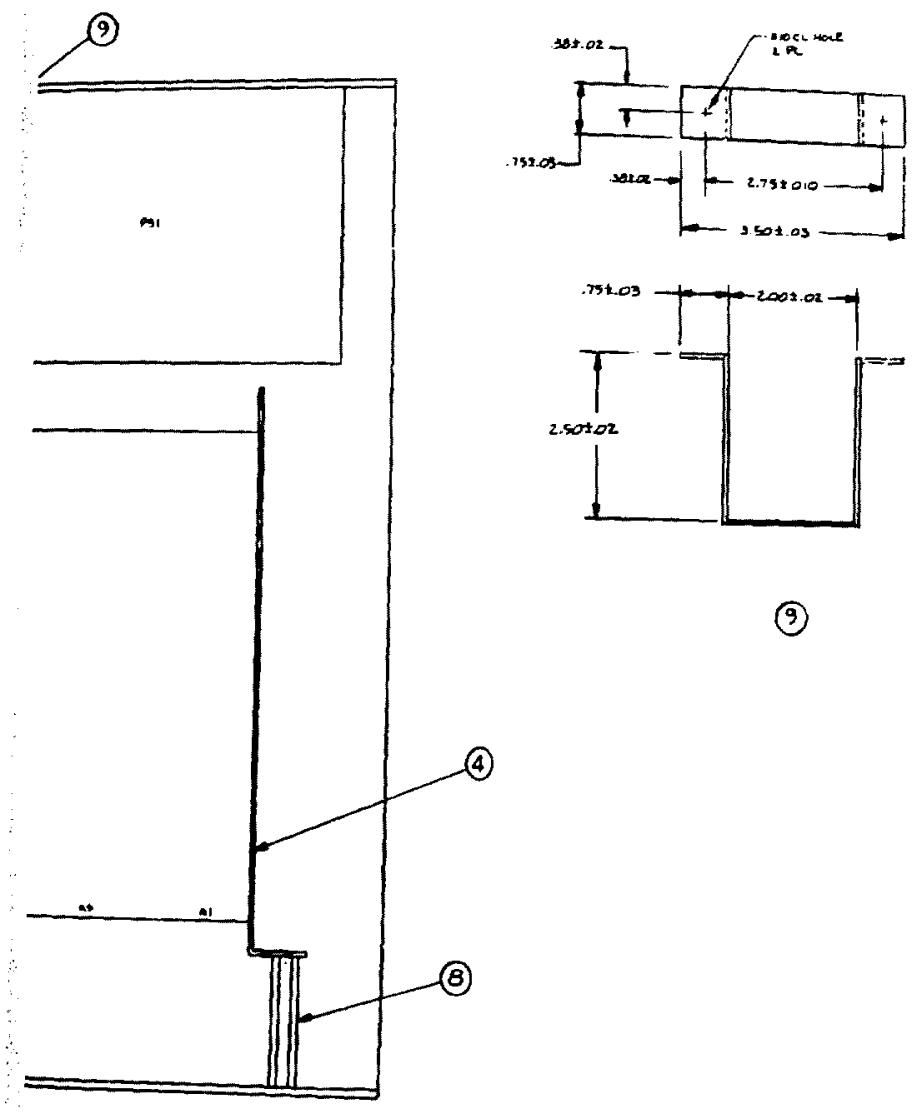

5. (continued)

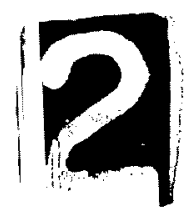


APPENDIX B--PROGRAM FLOW CHART 

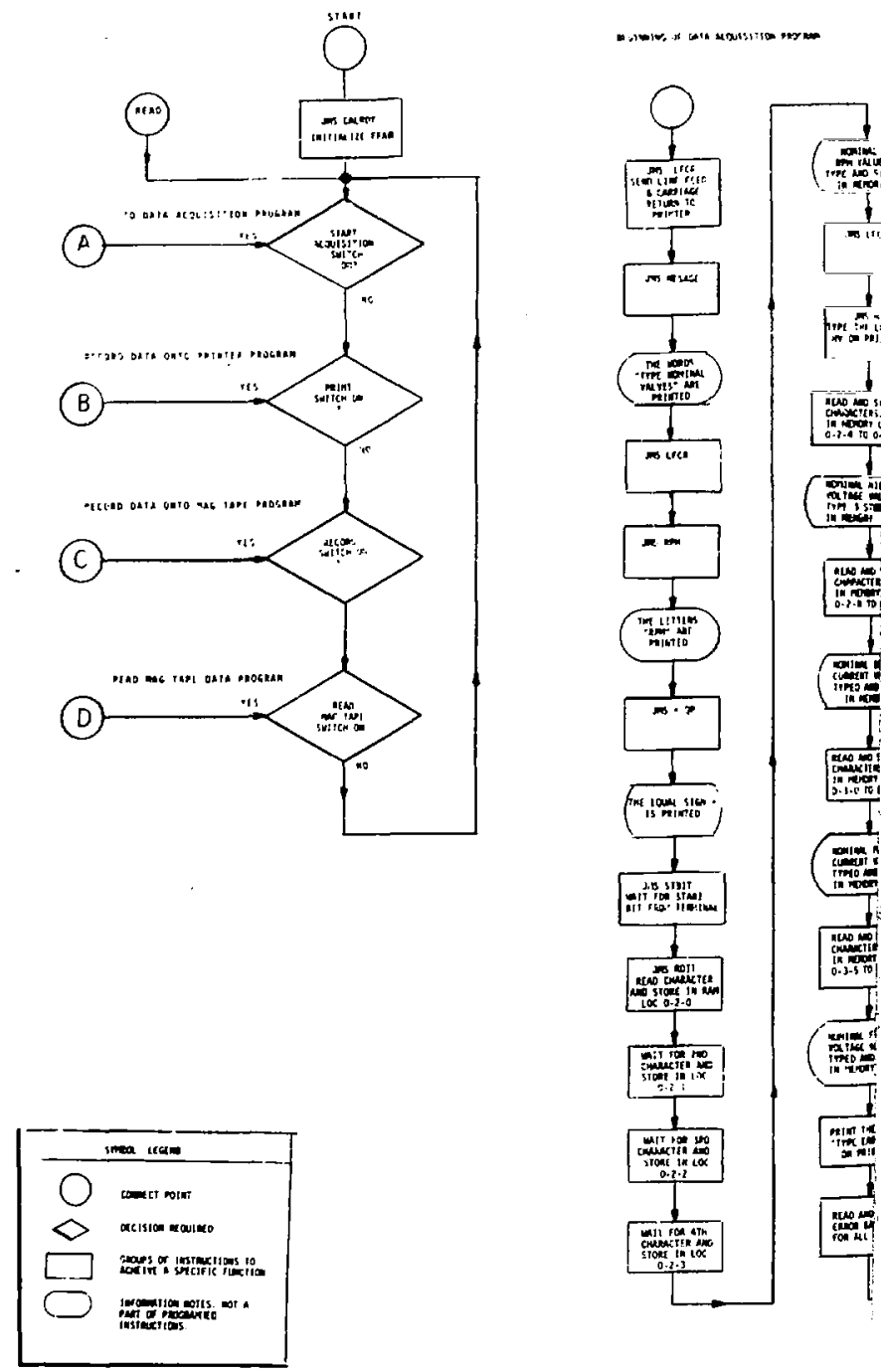

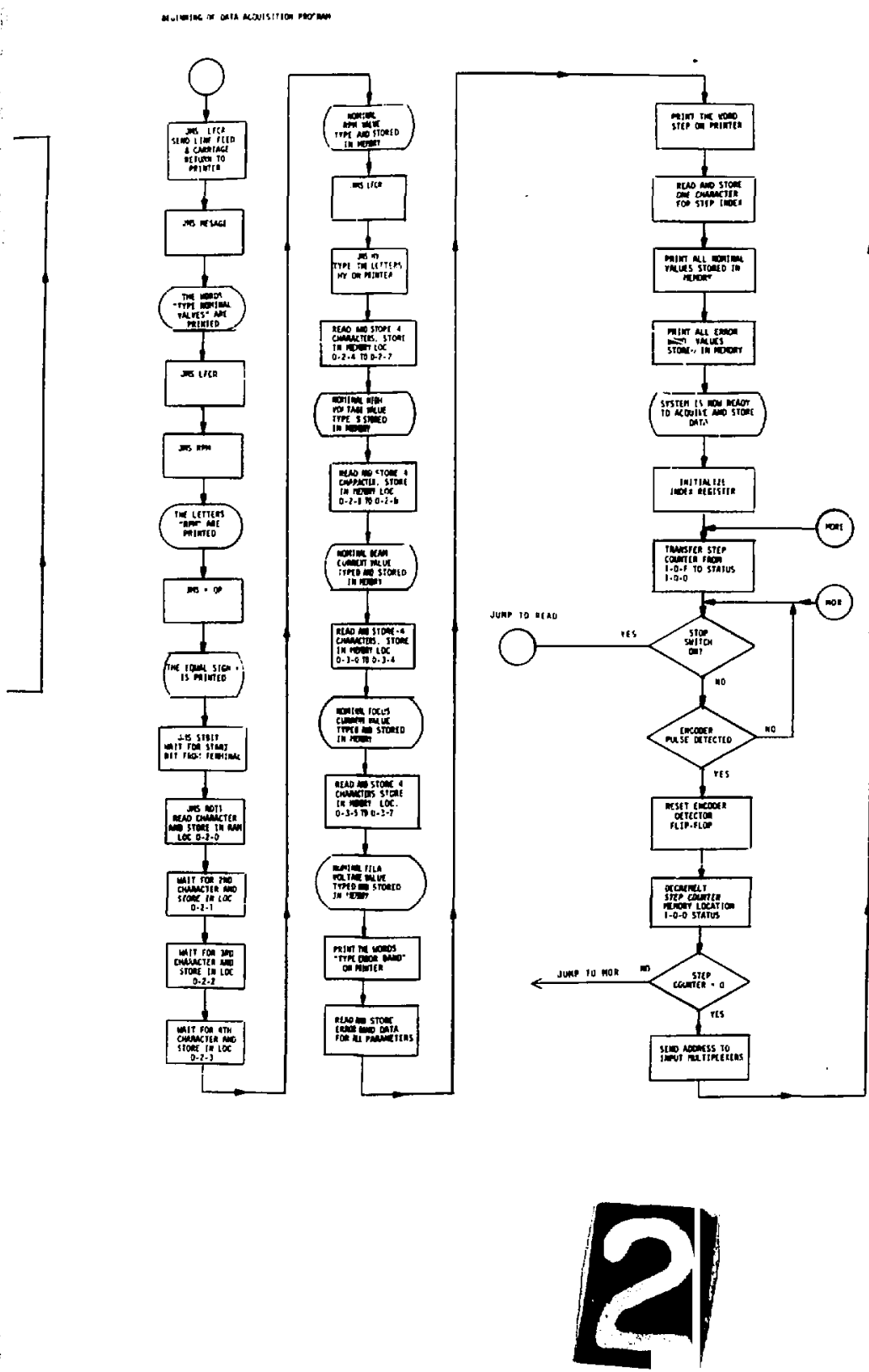


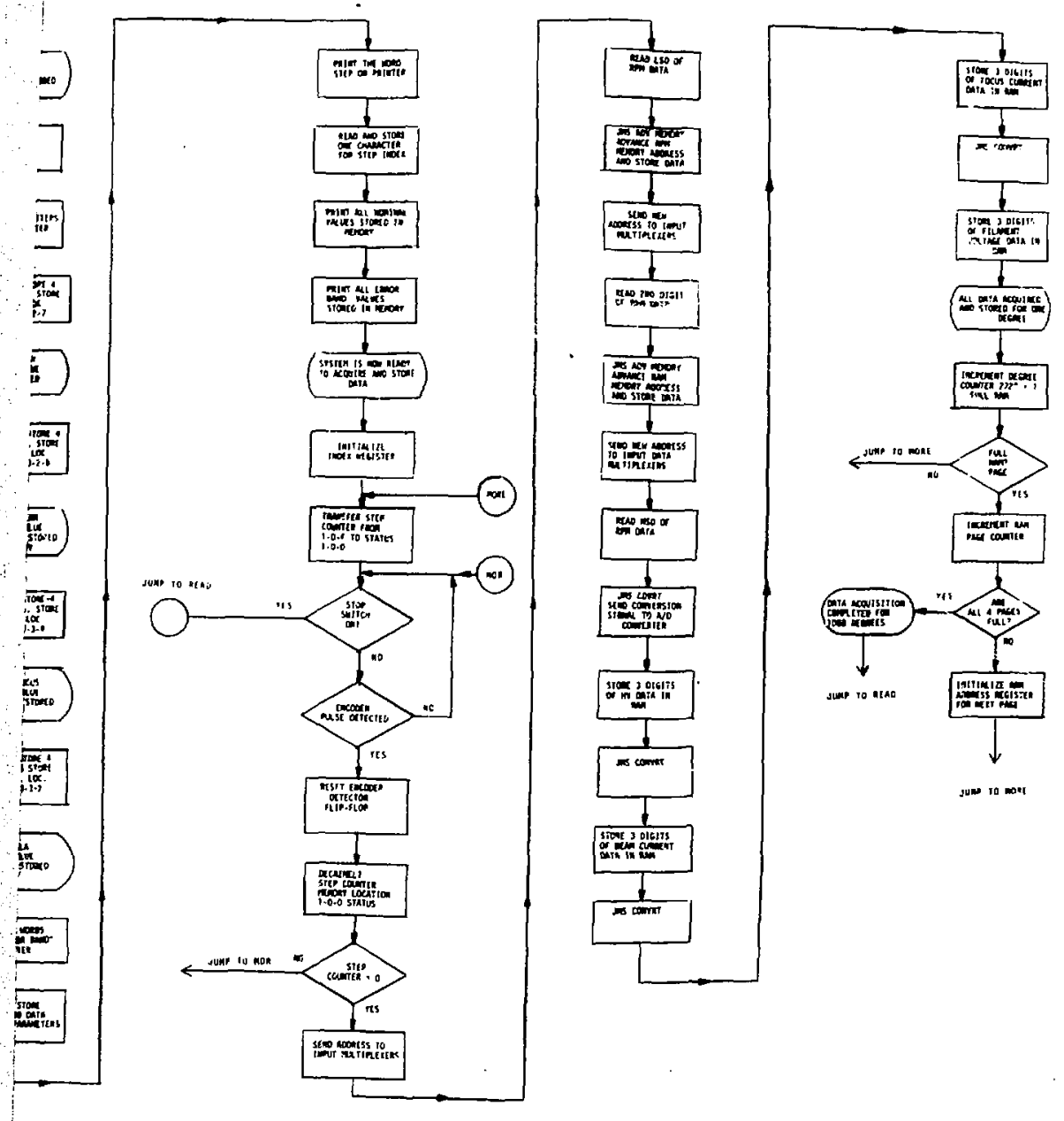



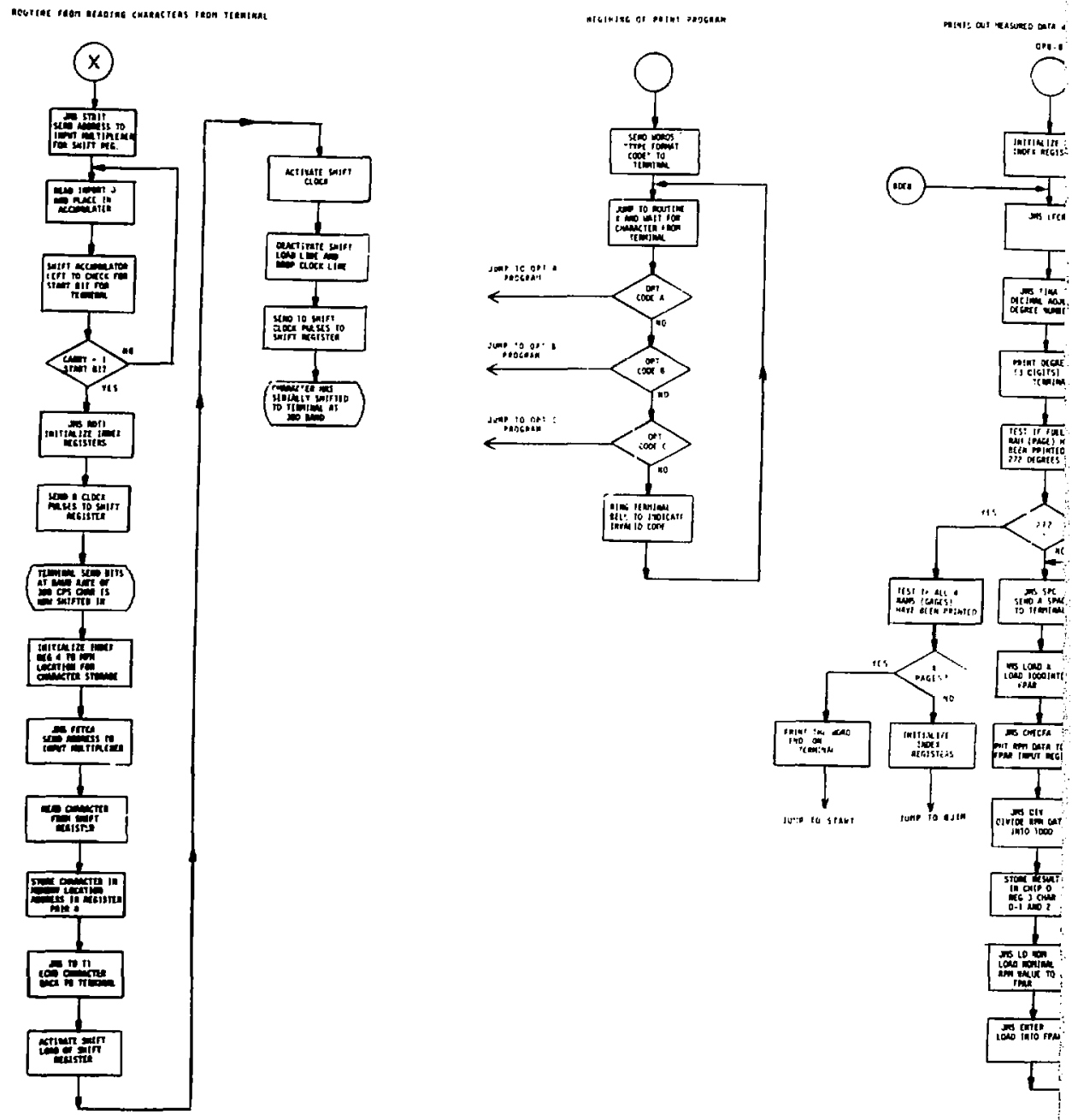

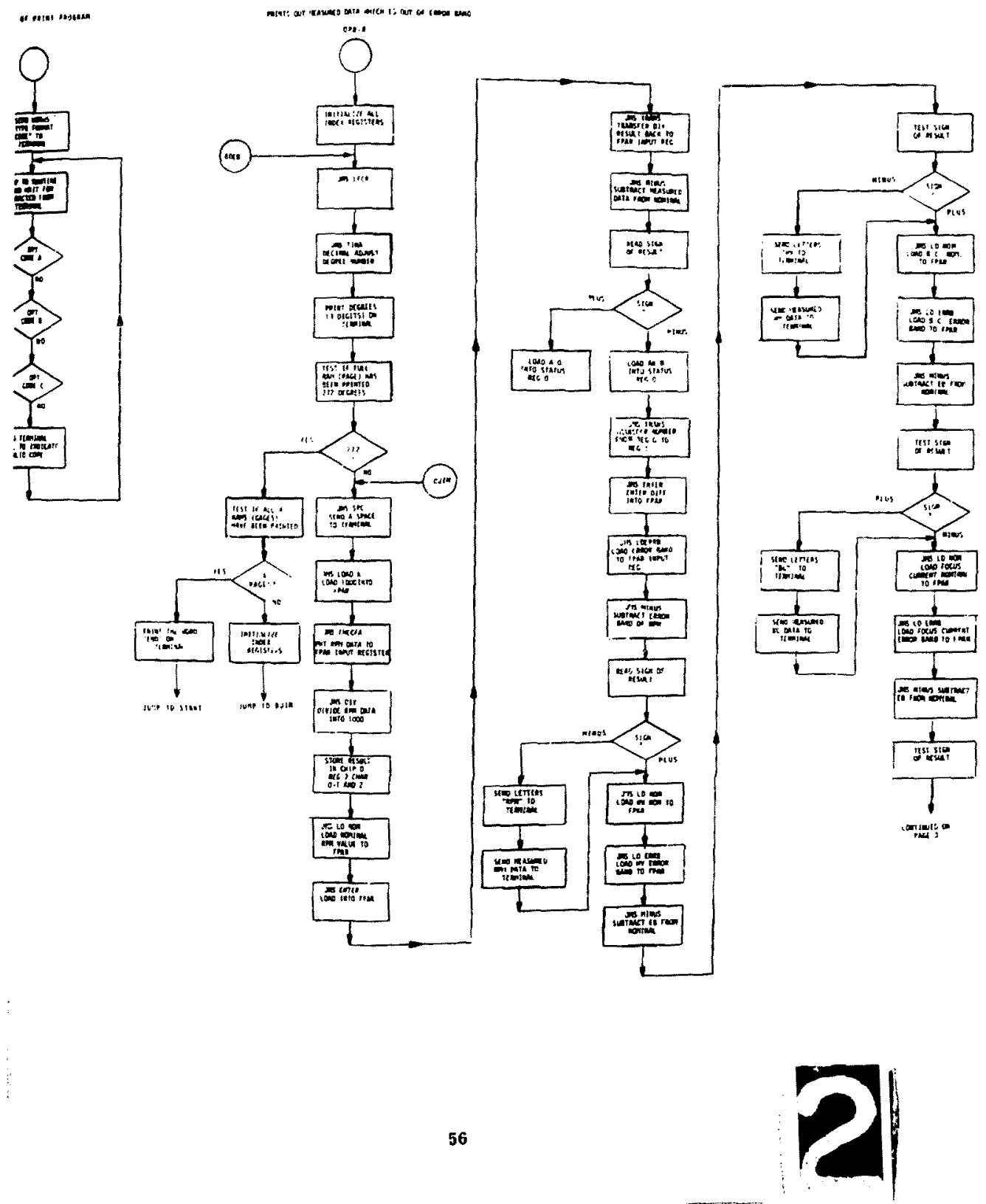


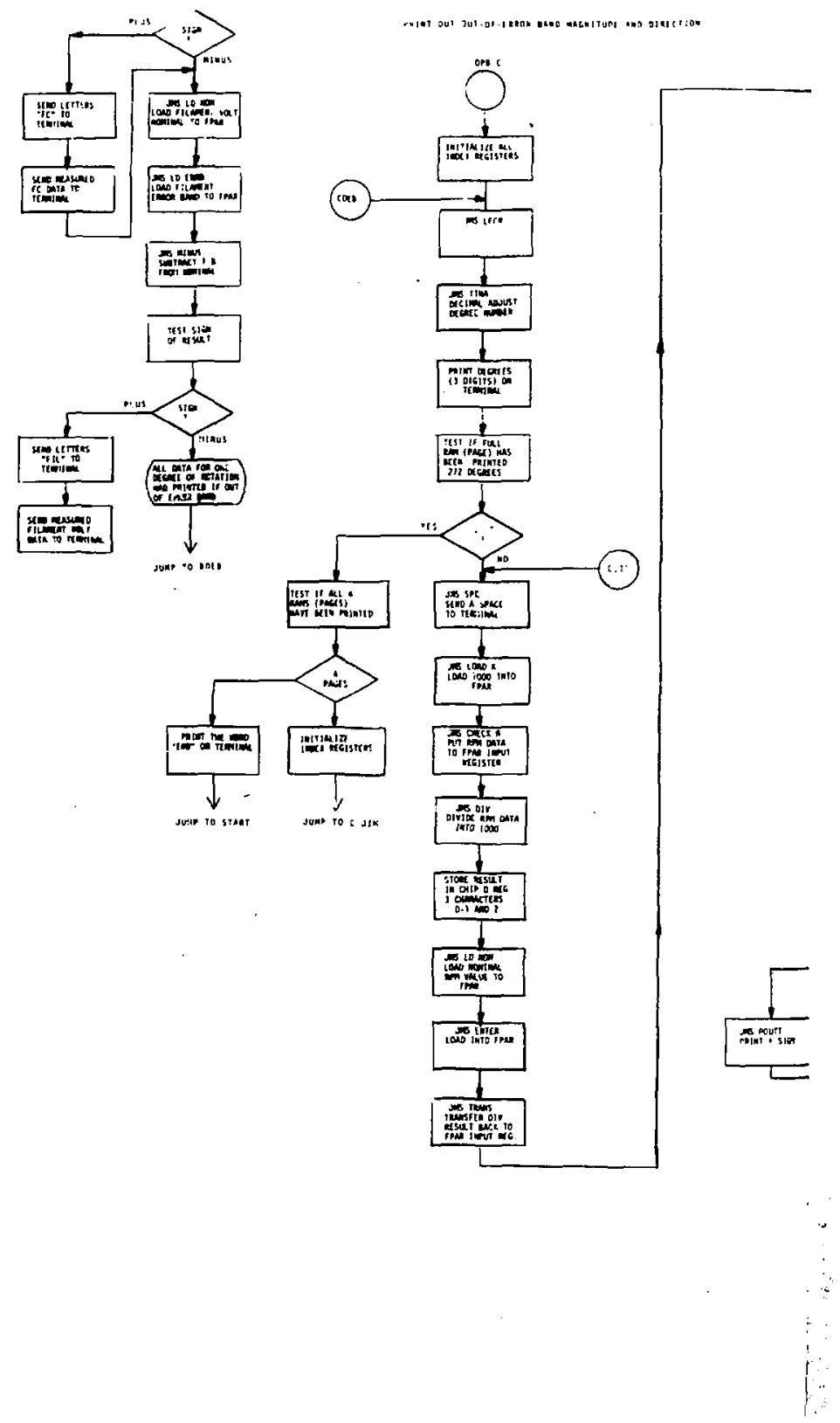




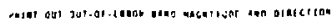
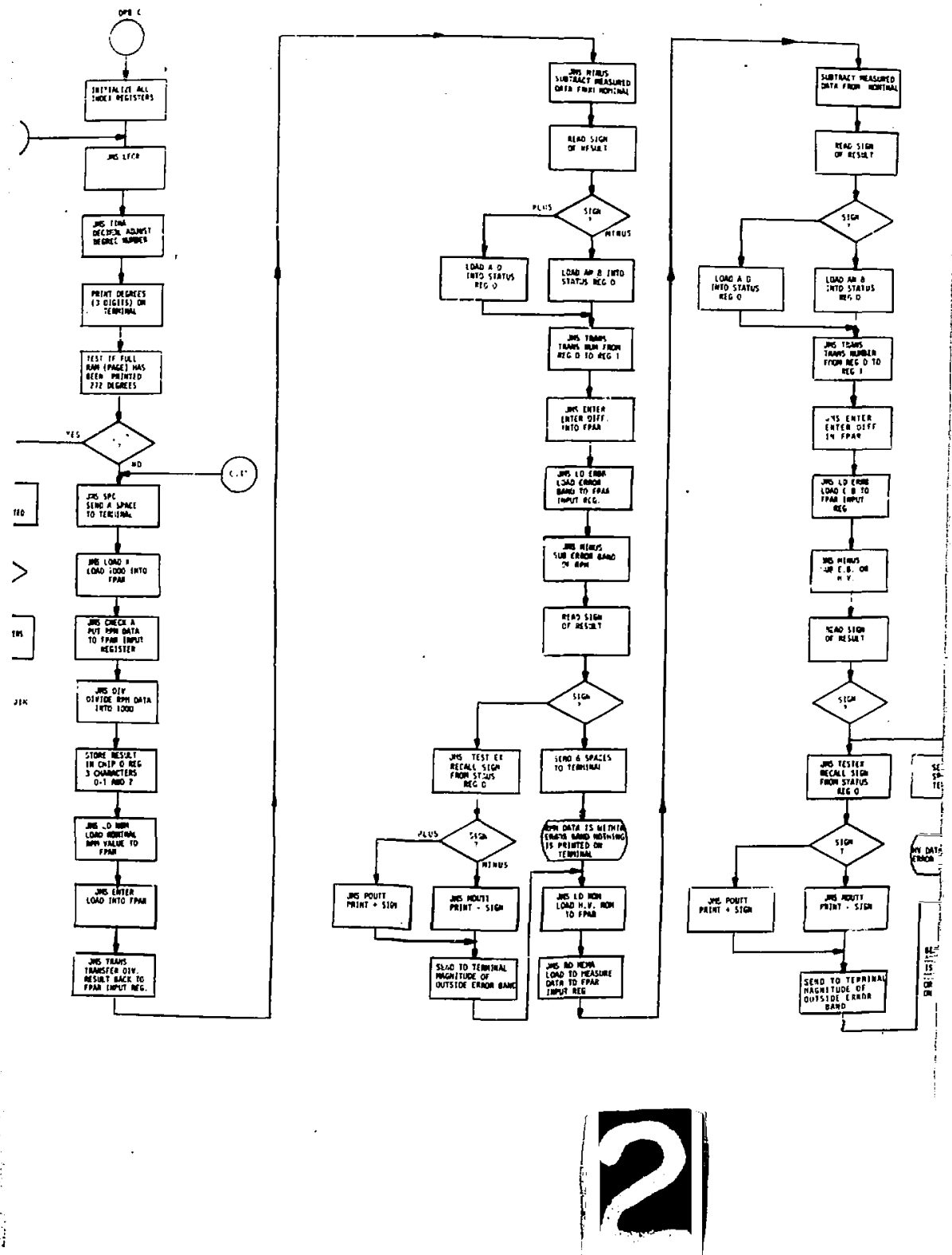

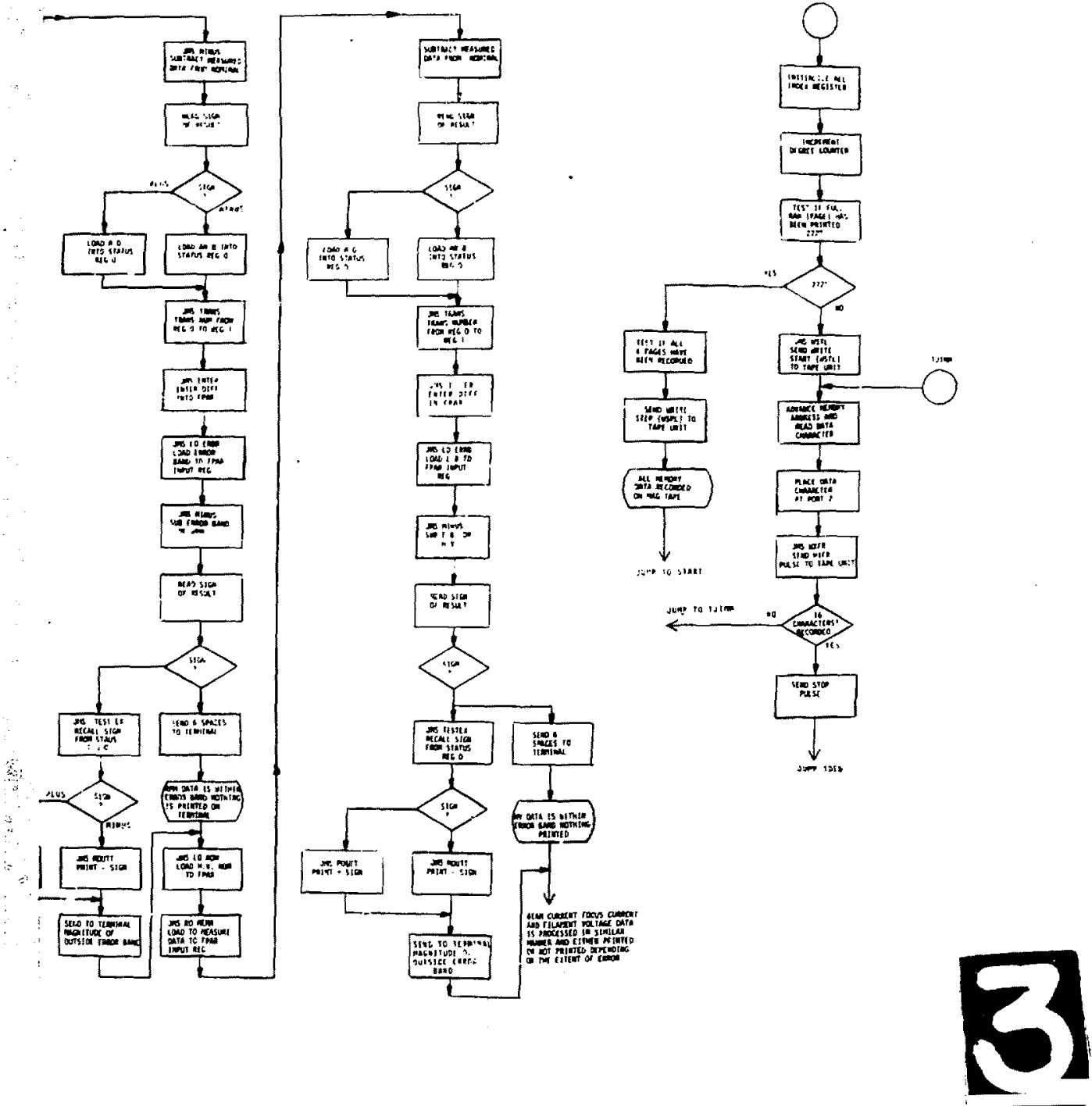

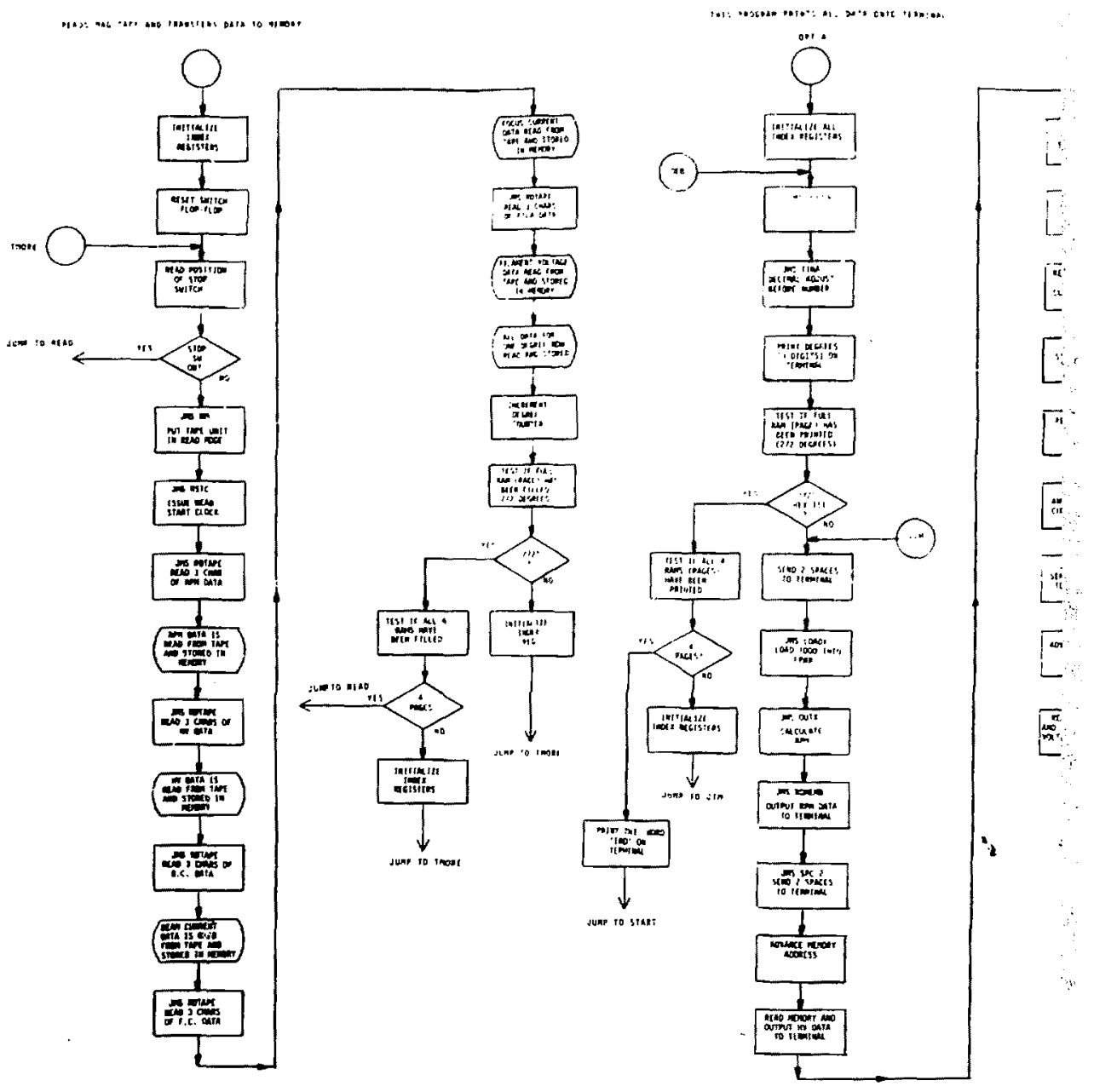

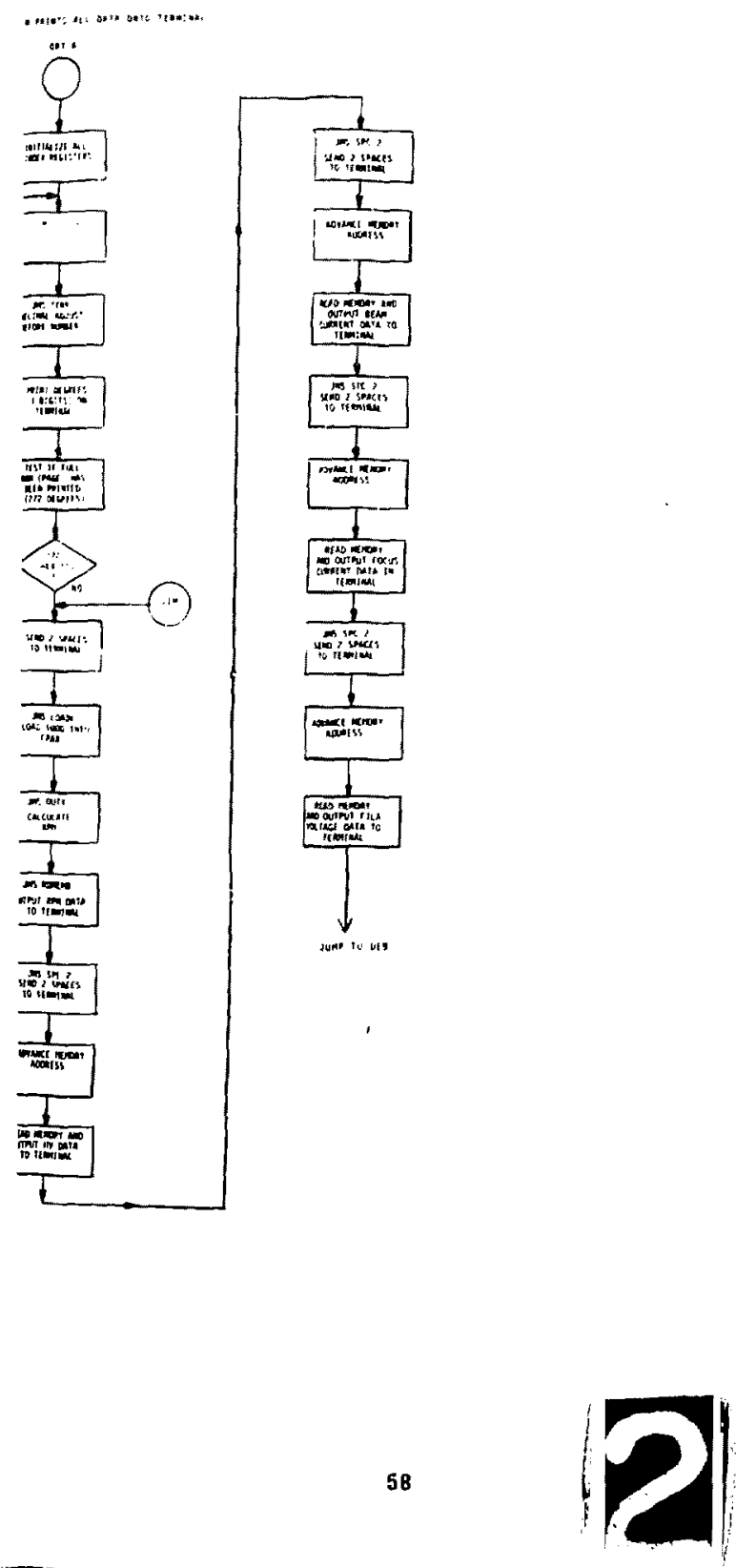
APPENDIX C--DETAILED SCHEMATICS 


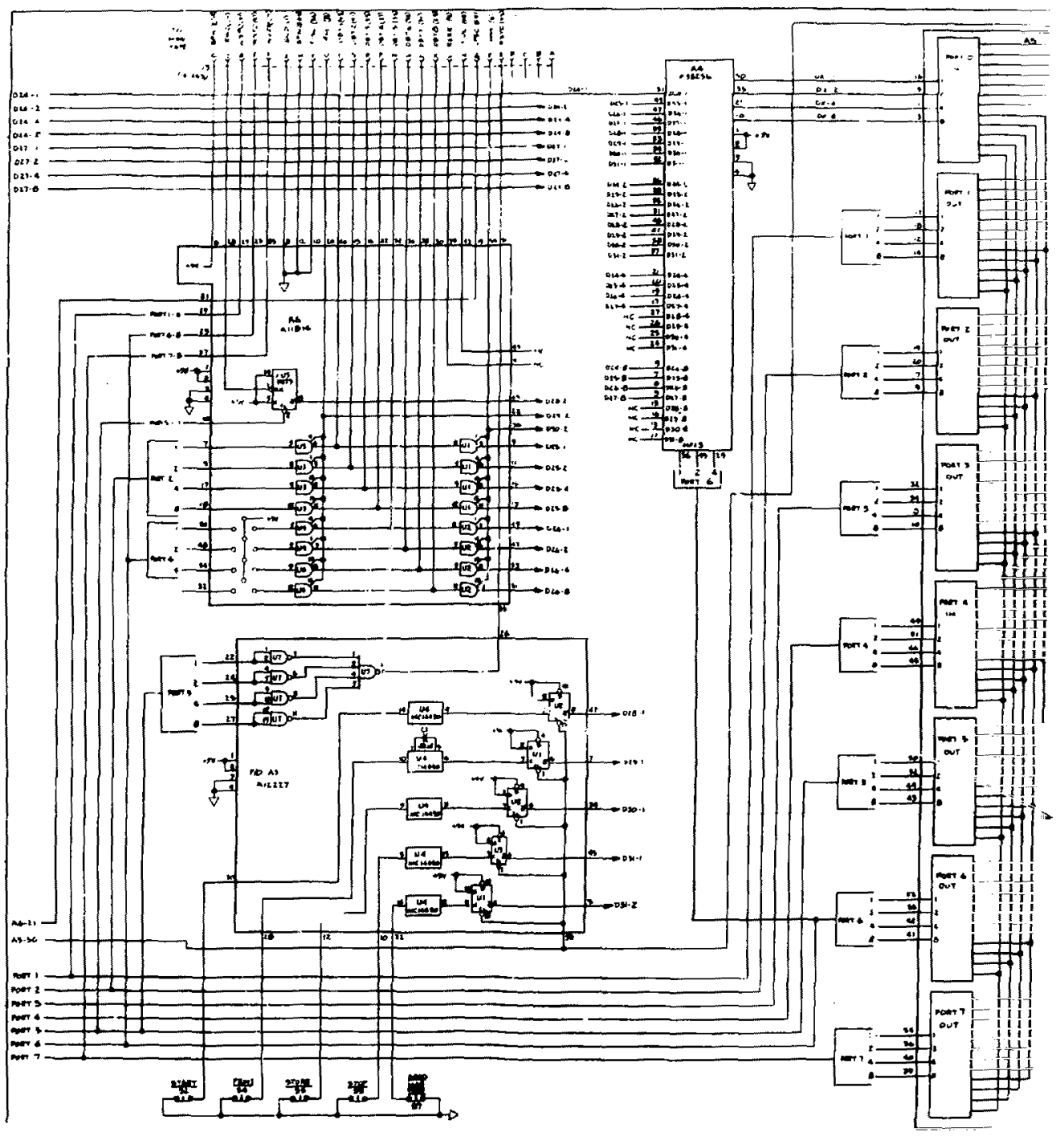

Electron Beam Welder Data Acquisition Systern 


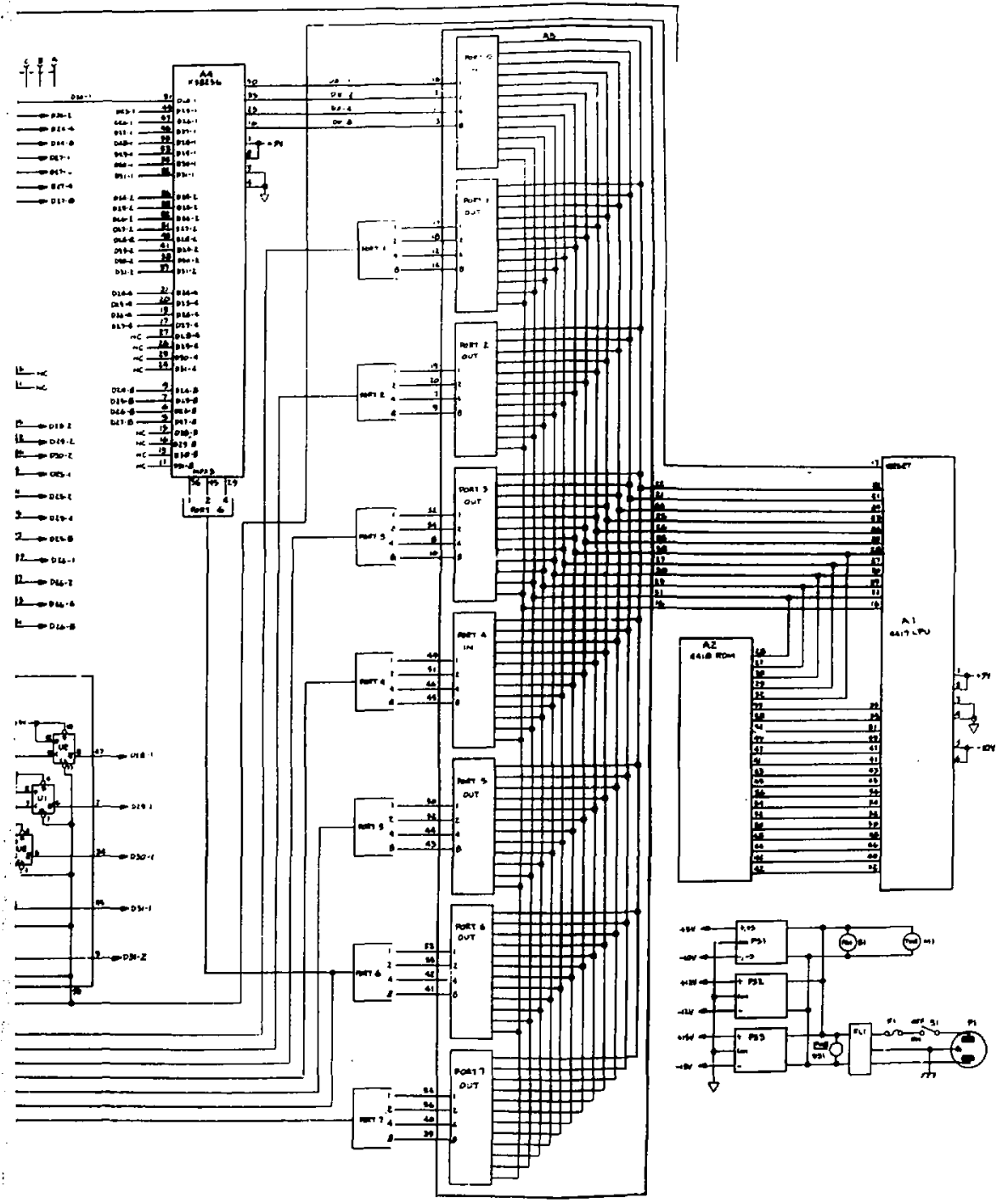

3ctron Beam Welder Data Acquisition System

4

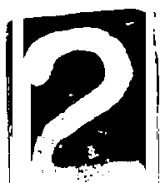




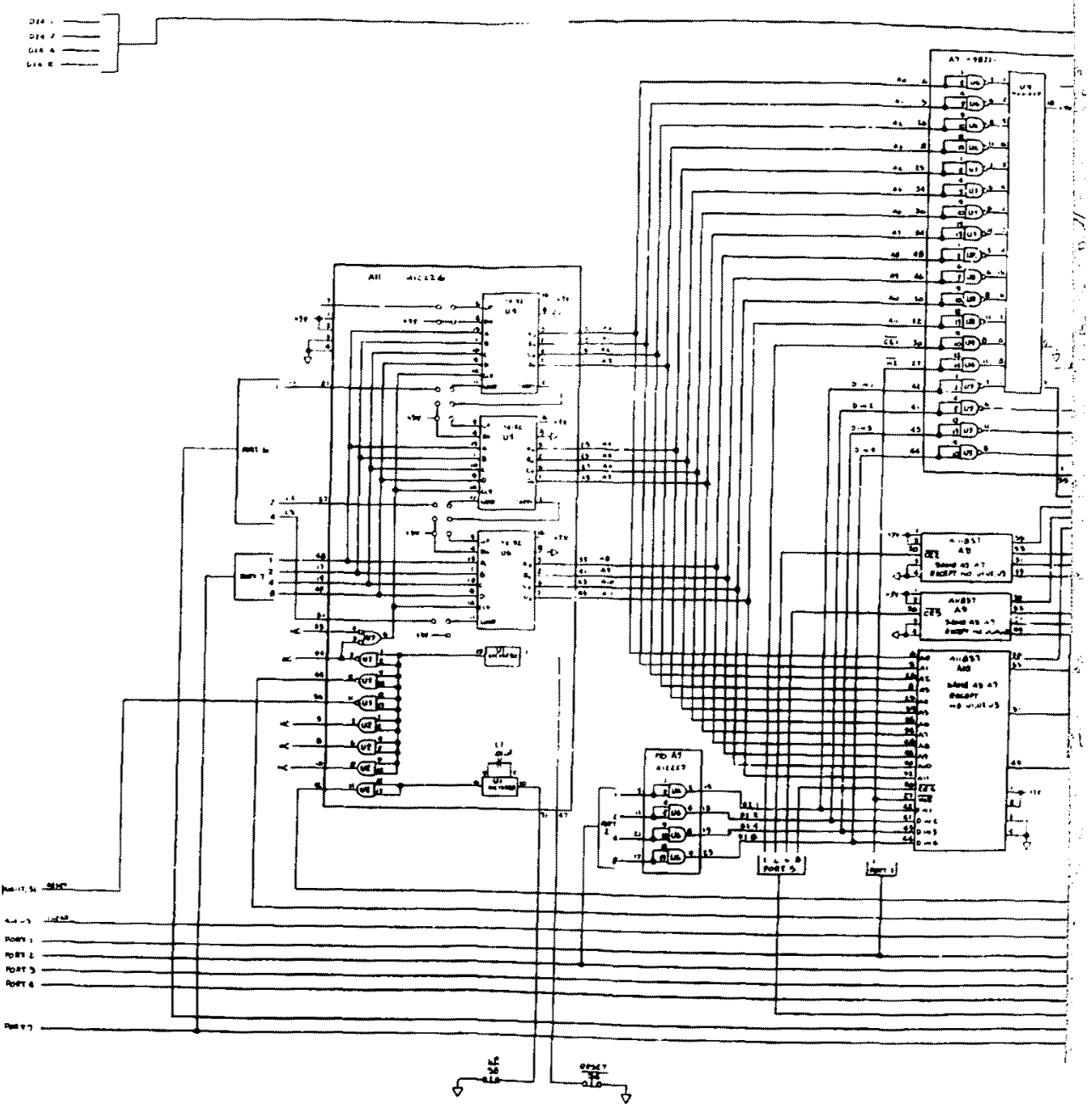




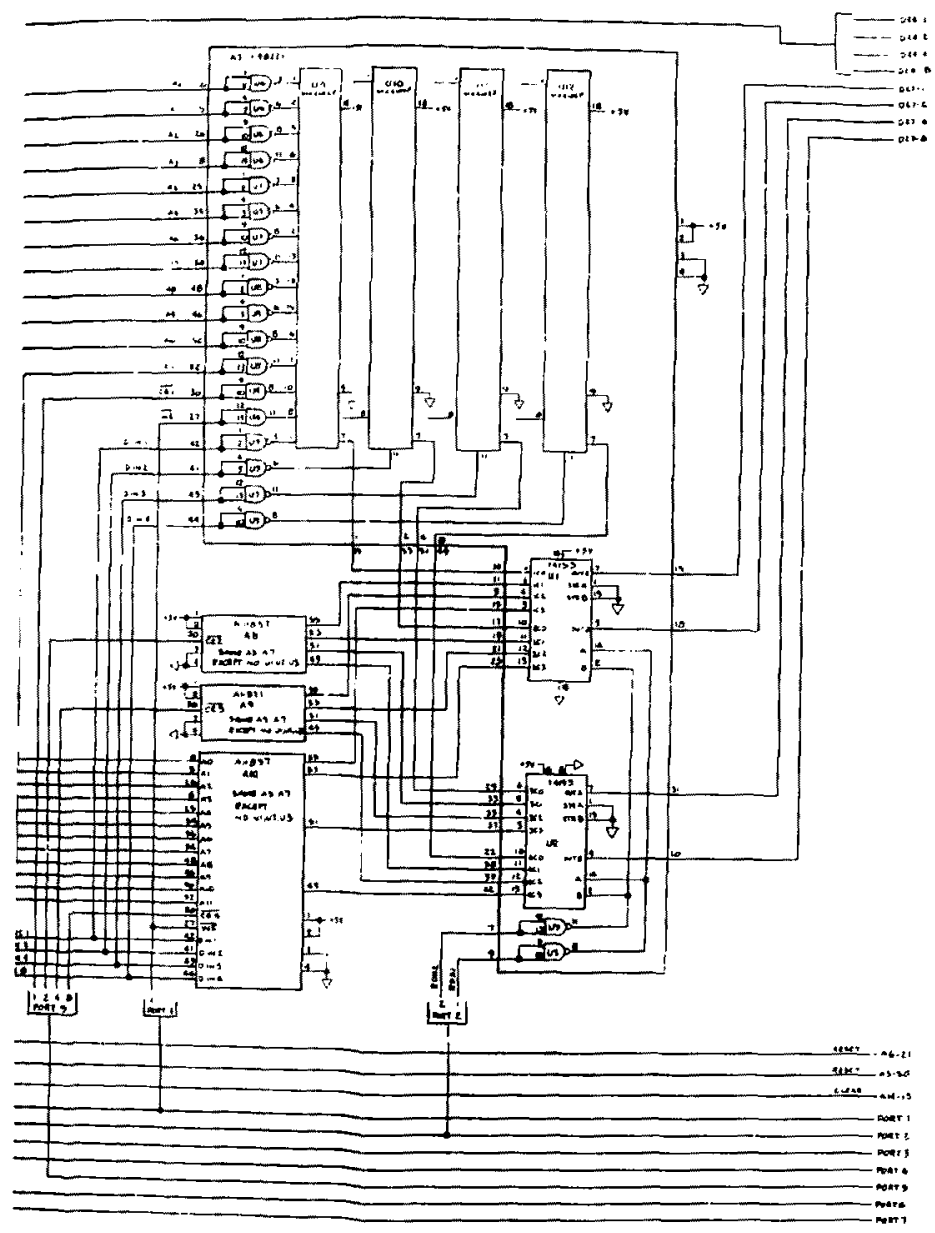




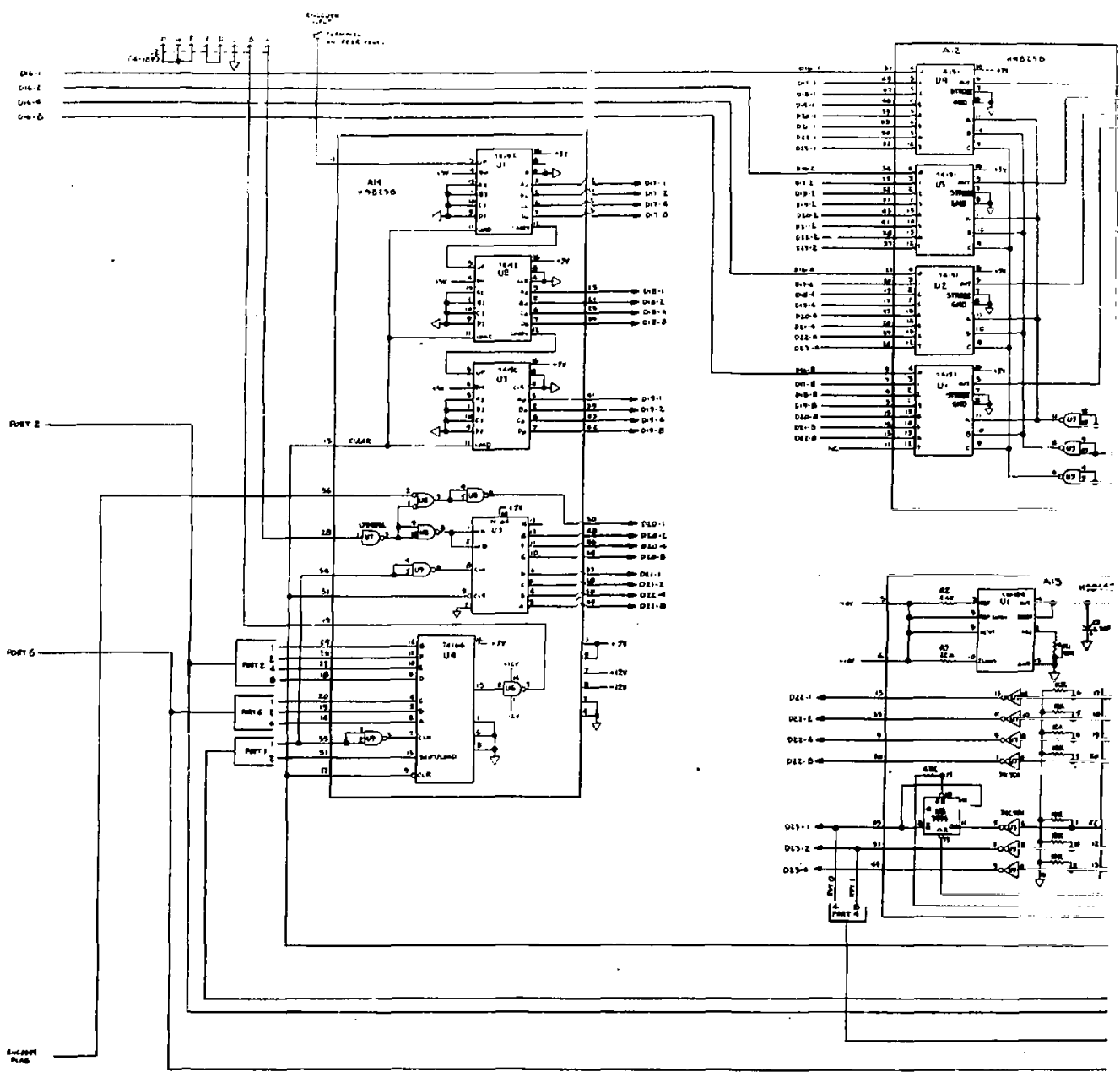



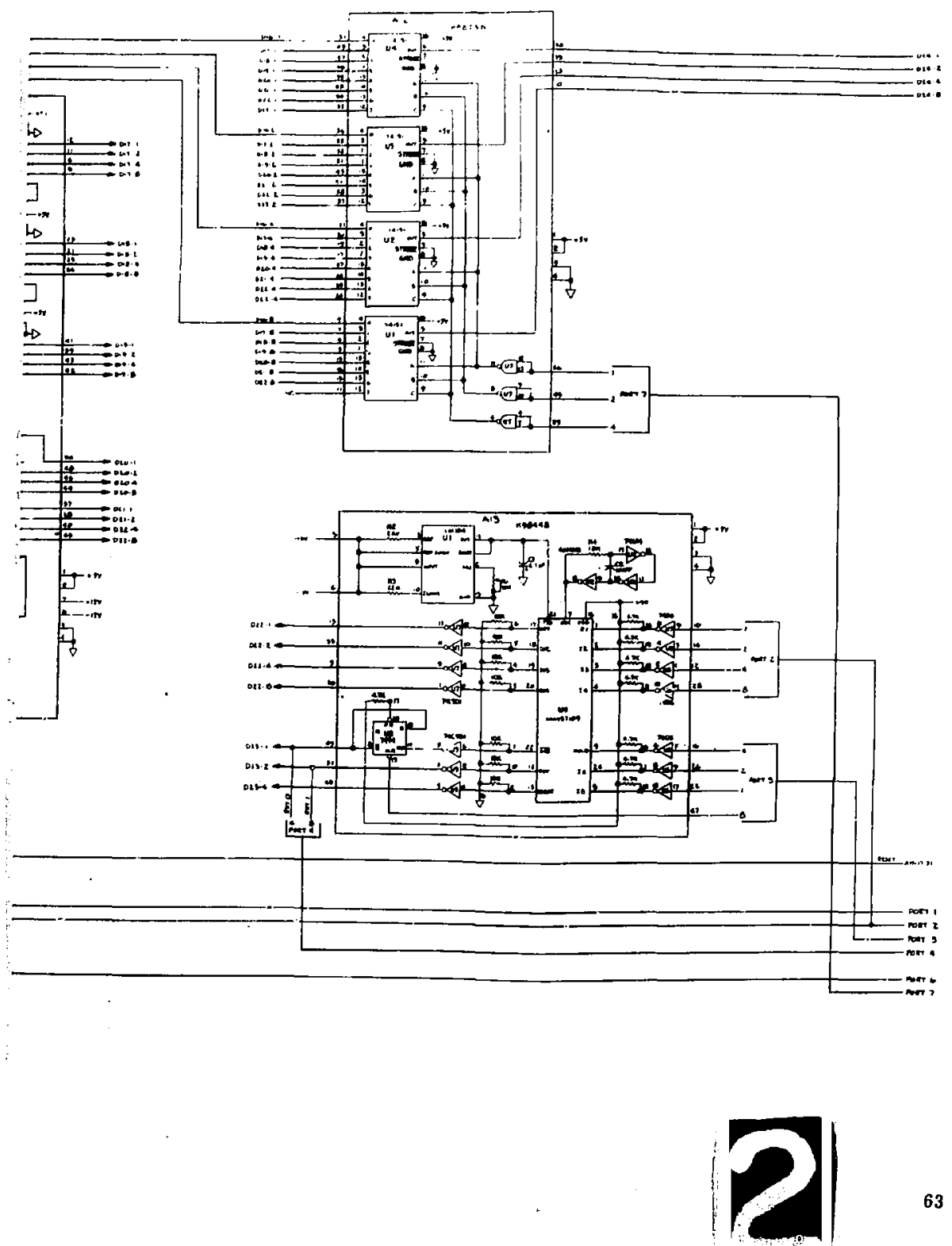


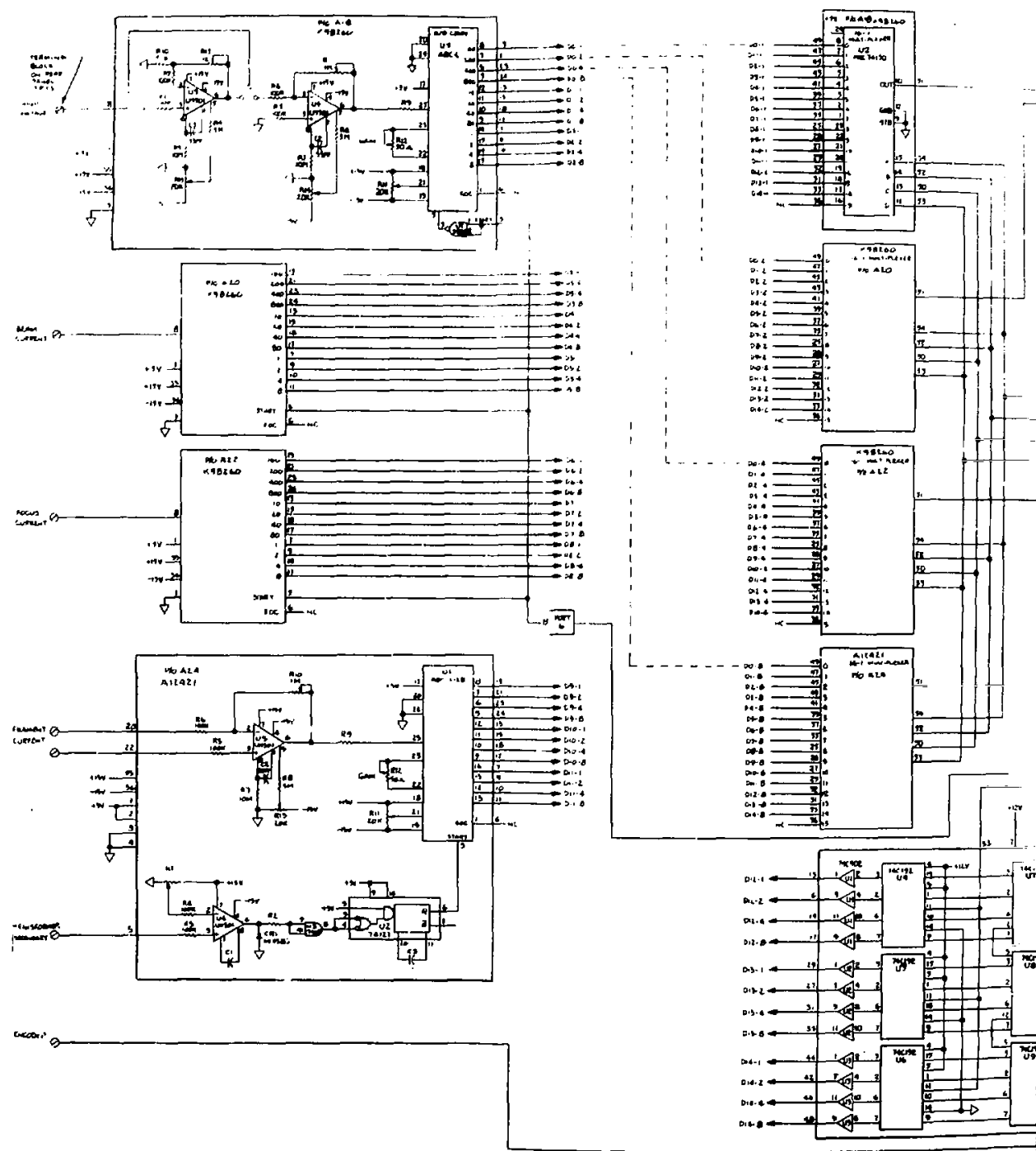




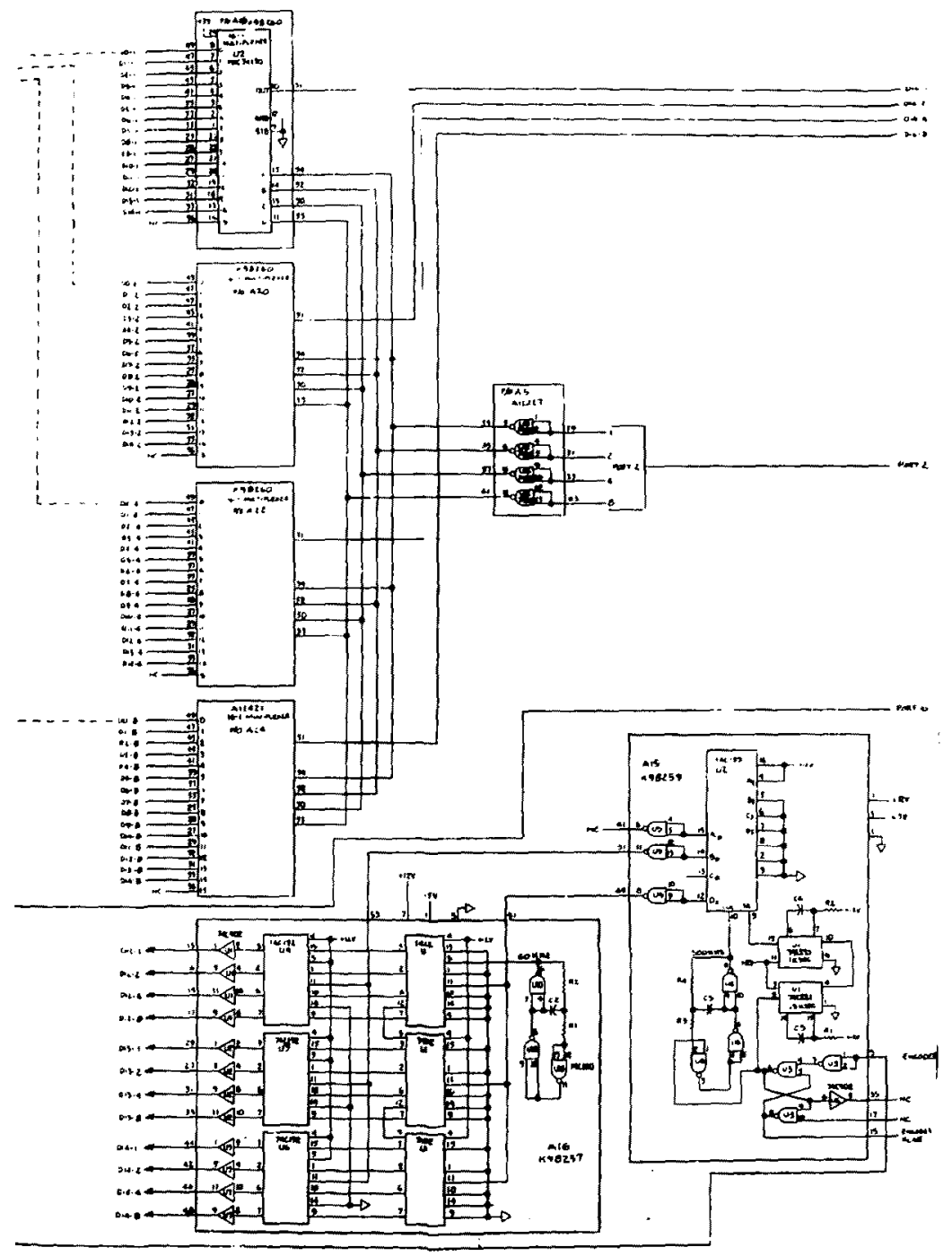

